NIST Technical Note 2210

\title{
Inflow Turbulence Effects on Large Eddy Simulations of the Flow around an Axisymmetric Hill
}

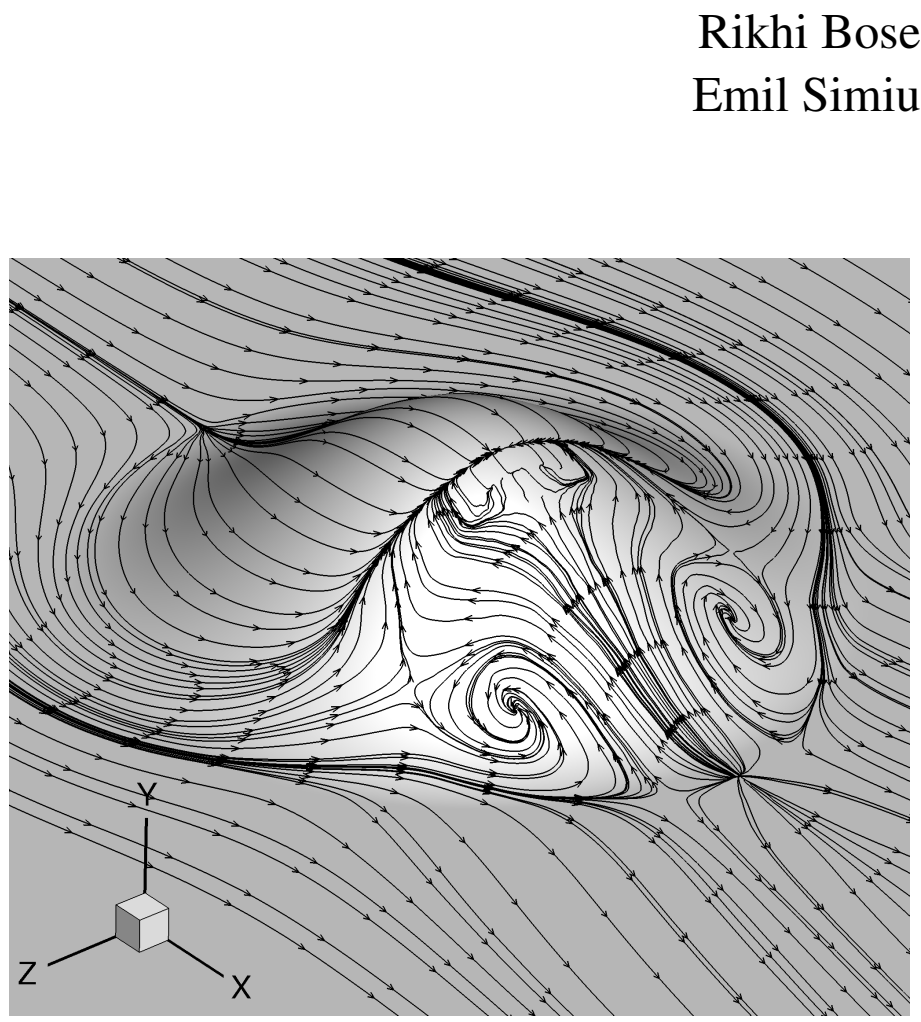

This publication is available free of charge from: https://doi.org/10.6028/NIST.TN.2210

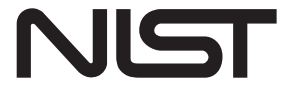

National Institute of Standards and Technology U.S. Department of Commerce 
NIST Technical Note 2210

\title{
Inflow Turbulence Effects on Large Eddy Simulations of the Flow around an Axisymmetric Hill
}

\author{
Rikhi Bose \\ Emil Simiu \\ Materials and Structural Systems Division \\ Engineering Laboratory
}

This publication is available free of charge from:

https://doi.org/10.6028/NIST.TN.2210

March 2022

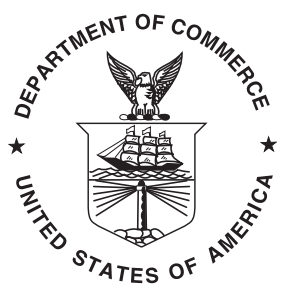

U.S. Department of Commerce Gina M. Raimondo, Secretary

National Institute of Standards and Technology James K. Olthoff, performing the non-exclusive functions and duties of the Under Secretary of Commerce for Standards and Technology and Director, National Institute of Standards and Technology 
Certain commercial entities, equipment, or materials may be identified in this document in order to describe an experimental procedure or concept adequately. Such identification is not intended to imply recommendation or endorsement by the National Institute of Standards and Technology, nor is it intended to imply that the entities, materials, or equipment are necessarily the best available for the purpose.

National Institute of Standards and Technology Technical Note 2210

Natl. Inst. Stand. Technol. Tech. Note 2210, 61 pages (March 2022)

CODEN: NTNOEF

This publication is available free of charge from: https://doi.org/10.6028/NIST.TN.2210 


\begin{abstract}
Turbulent flow around an axisymmetric hill at Reynolds number $R_{H} \approx 1.3 \times 10^{5}$ with respect to the hill height is studied using resolved large-eddy simulations (LES). The purpose of the paper is to examine the effect of turbulence in the inflow boundary condition on the flow around the hill. To this end LES simulations were performed for the following inflow boundary conditions: (i) mean turbulent boundary-layer profile obtained experimentally, and (ii) unsteady turbulent velocity profiles from a precursor LES, with the mean profile of the precursor LES forced to agree with experimental results, all other flow parameters in the two simulations being identical. Comparisons with reliable experiments available in the literature showed that the flow, in particular the complex separation dynamics in the lee-side of the hill, is sensitive to the inflow boundary condition. It was found that the mean flow and turbulence statistics in the turbulent-inflow simulation are in good agreement with the experimental results both upstream and downstream of the hill. However, the steady-inlet simulation yields a thicker and longer separation region than was observed experimentally. Consequent recovery of the flow downstream is also delayed where an inner vortex rotating in the same direction accompanies an outer vortex in either side of the midspan plane; the inner vortex pair is not obtained in either the experiment or the turbulent-inflow simulation. Despite these significant discrepancies, the upstream-side flow and the separation location for both simulations are in reasonably good agreement with the experiment. This can be attributed to the fact that the pressure gradient set by the geometry dictates the separation initiation through the formation of an internal layer. Incoming turbulence in a simulation suppresses the separated shear layer by increasing the near-wall velocity gradient, thereby causing early reattachment as in the experiment.
\end{abstract}

\title{
Key words
}

Axisymmetric hill; Complex topography; large-eddy simulation (LES); Turbulence. 


\section{Table of Contents}

1 Introduction 1

2 Numerical experiment setup $\quad 5$

2.1 Large-eddy simulations \& Sub-grid scale modeling 6

2.2 Flow solver 7

2.3 Computational grid $\quad 7$

$\begin{array}{ll}2.4 \text { Boundary conditions } & 10\end{array}$

2.5 Calculations \& Sampling 10

2.6 Efficacy of the SGS model 11

3 Comparison with experiments $\quad 14$

3.1 Precursor LES corresponding to case $T \quad 14$

3.2 Results: Time-averaged flow 17

3.2.1 Pressure distribution 17

3.2.2 Lee-side separation \& reattachment 21

3.2.3 Flow recovery 29

3.3 Results: Instantaneous flow \& Spectra 34

4 Effect of inlet turbulence $\quad 40$

5 Discussion \& Conclusions $\quad 48$

References $\quad 51$

\section{List of Figures}

Fig. 1 Schematic of the setup for the numerical experiment.

Fig. 2 Grid resolutions (solid lines) in viscous wall unit at the inlet of the main LES domain in the (a) wall-normal, $(b)$ streamwise and spanwise directions. Grid stretching ratio (dashed lines) is also shown for grids in all three directions. The dark and grey lines represent the corresponding curves for $x-$ and $z-$ grids, respectively. The $y-$ and $z$ - grids remained same for both precursor and main simulations.

Fig. 3 Grid resolution in local viscous wall units from the LES: $(a)-(c)$ case $S$ and $(d)-(f)$ case $T .(a)$ and $(d)$ streamwise resolution $(\Delta x+) ;(b)$ and $(e)$ wall-normal resolution at the centroid of the first wall-adjacent layer of cells $\left(\Delta y_{1 / 2}+\right) ;(c)$ and $(f)$ spanwise resolution $(\Delta z+)$.

Fig. 4 Ratio of the mean SGS eddy viscosity to the molecular viscosity $\left(\left\langle v_{S G S}\right\rangle / v\right)$ in the midspan $(z=0)$ plane for cases $S$ (top frame) and $T$ (bottom frame).

Fig. 5 Ratio of $(a)$ the total eddy viscosity to the molecular viscosity $\left(v_{T} / v\right)$ and (b) the mean SGS eddy viscosity to the total eddy viscosity $\left(\left\langle v_{S G S}\right\rangle / v_{T}\right)$ in the midspan $(z=0)$ plane for case $S$. 
Fig. 6 Ratio of (a) the total eddy viscosity to the molecular viscosity $\left(v_{T} / v\right)$ and (b) the mean SGS eddy viscosity to the total eddy viscosity $\left(\left\langle v_{S G S}\right\rangle / v_{T}\right)$ in the midspan $(z=0)$ plane for case $T$.

Fig. 7 Scaled wall-normal profiles of $(a)$ time-averaged streamwise velocity $\left(\langle u\rangle / U_{0}\right)$ (b) turbulent kinetic energy and principal Reynolds stresses from the precursor simulation compared with the experimental results in [6].

Fig. 8 Frequency spectra of the fluctuating velocity components at three wall-normal heights $y+\approx 15,150$ and 1500 calculated in wall unit.

Fig. 9 Spanwise autocorrelation of the fluctuating streamwise velocity at three wallnormal heights $y+\approx 15,150$ and 1500 calculated in wall unit.

Fig. 10 Contours of the pressure coefficient $\left(C_{p}\right)$ plotted in the horizontal $(x, z)-$ plane, from experiment $[3,38]$ (top frame), from case $S$ (middle frame), and from case $T$ (bottom frame). The circles in the frames show the outer circumference of the hill geometry.

Fig. 11 Pressure coefficient $\left(C_{p}\right)$ along the $x$-axis in the midspan $(z=0)$ plane compared with experimental data [3, 38] and another high-resolution LES (the $L L$ simulation in [16]).

Fig. 12 Contour of the zero-mean streamwise velocity $\langle u\rangle=0$ and the inplane velocity vectors in the midspan $(z=0)$ plane in experiment [6] (top frame) and from current LESs (bottom frames).

Fig. 13 Streamlines based on the mean skin-friction on the lower wall projected on to the $(x, z)$-plane depicting the near-wall flow topology from (a) case $S$ and (b) case $T$. The filled red (blue) circles indicate nodes (saddles).

Fig. 14 Streamlines based on the mean velocity field depicting the flow topology at different wall-parallel planes at selected heights w.r.t. the bottom wall, determined from the experiment [6].

Fig. 15 Streamlines from in-plane mean velocity fields at wall-parallel planes at selected heights w.r.t. the bottom wall projected onto the $(x, z)$-plane obtained from the current LES: $(a)-(d)$ case $S$ and $(e)-(h)$ case $T$. The wall-normal heights of the selected planes are shown in inflow wall unit.

Fig. 16 Contours of the turbulent kinetic energy scaled by the reference velocity $\left(k / U_{0}^{2}\right)$ in the midspan $(z=0)$ plane from the experiment [6] (top frame), and from current LES: case $S$ (bottom left) and case $T$ (bottom right).

Fig. 17 Isosurfaces of the mean streamwise vorticity $\left(\left\langle\omega_{x}\right\rangle \frac{H}{U_{0}}\right)$ shown in the lee-side of the hill from the current LES case $S:(a)\left\langle\omega_{x}\right\rangle \frac{H}{U_{0}}= \pm 1$ and $(b)\left\langle\omega_{x}\right\rangle \frac{H}{U_{0}}=$ \pm 5 .

Fig. 18 Isosurfaces of the mean streamwise vorticity $\left(\left\langle\omega_{x}\right\rangle \frac{H}{U_{0}}\right)$ shown in the lee-side of the hill from the current LES case $T:(a)\left\langle\omega_{x}\right\rangle \frac{H}{U_{0}}= \pm 1$ and $(b)\left\langle\omega_{x}\right\rangle \frac{H}{U_{0}}=$ \pm 5 .

Fig. 19 Contours of mean streamwise vorticity $\left(\left\langle\omega_{x}\right\rangle \frac{H}{U_{0}}\right)$ plotted in wall-normal $(y, z)-$ planes at chosen streamwise locations from the LES cases: (a) and (c) $S$ and (b) and $(d) T$. The streamwise locations are $x / H=1.5$ and 2.5, respectively. 
Fig. 20 Contours of $T K E$ in the cross-stream $(y, z)$-plane at $x / H \approx 3.63$ from the experiment [38] and from current LES cases $S$ and $T$. Secondary flow is also depicted by showing the streamlines from the in-plane mean velocity vectors.

Fig. 21 Friction velocity in the spanwise direction in the flow recovery region at $x / H \approx 3.63$ from current LESs compared with the experiment [38].

Fig. 22 Wallnormal profiles of the mean $(a)$ streamwise $\left(\langle u\rangle / U_{0}\right)$ and $(b)$ spanwise $\left(\langle w\rangle / U_{0}\right)$ velocities at $x / H=3.63$ from current LES case $S$ (dashed lines) and case $T$ (solid lines) compared with experiment (symbols) [4]. For clarity, each profile of $\langle u\rangle / U_{0}$ and $\langle w\rangle / U_{0}$ has been shifted by the nondimensional units 1 and 0.25 , respectively.

Fig. 23 Wallnormal profiles of the scaled (a) TKE $\left(k / U_{0}^{2}\right)$ and $(b)$ resolved turbulent stress component $\left(\left\langle u^{\prime} v^{\prime}\right\rangle / U_{0}^{2}\right)$ at $x / H=3.63$ from current LES case $S$ (dashed lines) and case $T$ (solid lines) compared with experiment (symbols) [4]. For clarity, each profile of $k / U_{0}^{2}$ and $\left\langle u^{\prime} v^{\prime}\right\rangle / U_{0}^{2}$ has been shifted by nondimensional units 0.04 and 0.015 , respectively.

Fig. 24 Contours of instantaneous streamwise velocity fluctuation $\left(u^{\prime}\right)$ in extracted wall-parallel planes at chosen heights above the lower wall for the LES case $T$. The extracted planes are located at heights: $(a)-(d) y+\approx 150$, and $(e)-(h)$ $y+\approx 1500$ w.r.t. the viscous wall unit at inlet.

Fig. 25 Instantaneous streamlines from inplane velocity fields in an extracted wallparallel plane projected onto the $(x, z)$-plane as in the experimental results of Fig. 14, for current LES case $T$. The time instants are same as in Fig. 24. The circle shows the outer circumference of the hill. The plane is at a height $y+\approx 150$ (w.r.t. the viscous wall unit at inlet) above the bottom wall.

Fig. 26 Frequency spectra of the streamwise (left) and spanwise (right) velocity fluctuations in the midspan $(z=0)$ plane at $x / H=3.63$ compared with experiment (symbols): (a)-(b) $y / H=0.36 ;(c)-(d) y / H=0.2 ;(e)-(f) y / H=$ 0.07 . The dashed lines correspond to $-5 / 3-$ law.

Fig. 27 Streamwise profiles of $(a)$ the hill surface, its slope $\left(\frac{\partial y}{\partial x}\right)$, and curvature $\kappa=$ $\frac{\left|\frac{\partial^{2} y}{\partial x^{2}}\right|}{\left[1+\left(\frac{\partial y}{\partial x}\right)^{2}\right]^{1.5}},(b)$ the streamwise pressure gradient $\left(H \frac{\partial C_{p}}{\partial x}\right)$ on the bottom wall (c) displacement thickness $(\delta *)$ and momentum thickness $(\theta)$ at the bottom wall in midspan plane $(z / H=0)$.

Fig. 28 Local profiles of $\langle u\rangle / U_{0}$ (frames $a, c, e, g$ ) and $\langle v\rangle / U_{0}$ (frames $b, d, f, h$ ) components of the mean velocity in the midspan $(z / H=0)$ plane at indicated streamwise stations from the current LES case $S$ (dashed lines) and case $T$ (solid lines). The abscissa is the coordinate-shifted $y$ - axis adjusting for the hill profile. In frames showing profiles at more than one location, darker colors are used for farther downstream locations. 
Fig. 29 Local profiles of the resolved turbulent stresses: $x$ - component $\left(\frac{\left\langle u^{\prime} u^{\prime}\right\rangle}{U_{0}^{2}}\right)$ in frames $a, d, g, j, y$-component $\left(\frac{\left\langle v^{\prime} v^{\prime}\right\rangle}{U_{0}^{2}}\right)$ in frames $b, e, h, k$, and cross-stream component $\left(\frac{\left\langle u^{\prime} v^{\prime}\right\rangle}{U_{0}^{2}}\right)$ in frames $c, f, i, l$ in the midspan $(z / H=0)$ plane at the indiacted streamwise stations from the current LES case $S$ (dashed lines) and case $T$ (solid lines). The abscissa is the coordinate-shifted $y$ - axis adjusted for the hill profile. In frames showing profiles at more than one location, darker colors are used for farther downstream locations.

Fig. 30 Profiles of $(a)$ mean streamwise velocity $\left(\langle u\rangle / U_{0}\right)$ in Fig. 28(a,c,e), and $(b)$ resolved turbulent stress $\frac{\left\langle u^{\prime} u^{\prime}\right\rangle}{U_{0}^{2}}$ in Fig. 29(a,d,g) against coordinate-shifted $y-$ axis plotted in log scale at streamwise stations: $x / H=-2$ (red), $x / H=$ -1.65 (violet), -1.35 (green), -1.11 (blue), -1 (black), -0.48 (orange), and -0.26 (light blue) from the LES case $S$ (dashed lines) and case $T$ (solid lines).

Fig. 31 Contours of the production of turbulent kinetic energy scaled by the reference length and velocity scales $\left(p_{k} \frac{H}{U_{0}^{3}}\right)$ plotted in the midspan $(z / H=0)$ plane from the current LES: $(a)$ case $S$ and $(b)$ case $T$. Zero contour of the mean-streamwise velocity $(\langle u\rangle=0)$ is shown (solid line) to depict the mean recirculation region. Scaled streamwise velocity profiles $\left(\langle u\rangle / U_{0}\right)$ are also shown at $x / H=0.5,1,1.5$, and 2 by the coordinate-shifted dashed lines. The scale for $\langle u\rangle / U_{0}$ is shown at the top of the frame. 


\section{Introduction}

Both analytical and AI-based [1,2] approaches are currently being used to model complex flows. The amount of data obtained from experiments is typically insufficient for gaining in-depth physical insights and for enabling effective data-driven approaches. On the other hand, high-fidelity simulations, rigorously validated against experiments, can provide information that is not otherwise obtainable by any other means. In this work, large-eddy simulations (LES) of flow over a three-dimensional smooth-wall axisymmetric hill are performed with the primary goal of generating data for data-based turbulence modeling purposes. In the process of validating the results against experiments, intriguing insights are gained into the flow physics.

Flow over an axisymmetric three-dimensional hill is a canonical problem with a wide variety of complexities, including, among other flow features, flow accelerations and decelerations, pressure gradient and wall-curvature effects, distortion and yawing of the boundary layer in the upstream side of the hill, unsteady separation and reattachment in the wake, separated shear layers and their interaction with the external flow field and the recirculating flow, and flow recovery in the wake of the hill beyond reattachment. This study concerns flow around a $3-D$ smooth-wall axisymmetric hill, on which high-precision state-of-theart experimental techniques, including Laser-Doppler Velocimetry (LDV) and Hot-Wire Anemometry (HWA) have been used to obtain a detailed description of the flow [3-6]. For this study the ratio of the turbulent boundary-layer thickness to the hill height is approximately 0.5 , considerably larger than in [7]. The Reynolds number w.r.t. the hill height is $R e_{H}=\frac{U_{0} H}{v}=130,000$, and allows high fidelity simulations on modern super computers. The high-quality data from several wind-tunnel experiments performed for this flow being available for validation, it is widely used for testing turbulence models for their efficacy in capturing flow dynamics of the complex three-dimensional separation from curved surfaces.

Since the publication of the experimental results, several numerical efforts have been reported in, e.g., [8-17]. Reynolds-averaged Navier-Stokes (RANS) turbulence closure models have been used for the prediction of mean flows $[8,10,11,18,19]$. RANS simulations have predicted reasonably well the upstream mean flow and the pressure distributions [19]. However, they were unable to capture the lee-side mean separation flow dynamics, and have resulted in inadequate predictions of flows in recirculation regions and mean flows in the separation and flow recovery regions (e.g., $[8,11,18])$. High-resolution LESs in $[16,19]$, and also herein, show that the lee-side three-dimensional separational flow is intermittent and governed by large-scale vortical shedding of the scale of boundary layer thickness or hill height, possibly because of the approaching boundary layer being thin compared to the height of the hill. Because RANS models are formulated and tested for flows with turbulence scales much smaller than the mean flow distortion scales, perhaps thats is why almost all efforts with RANS have been unsuccessful in predicting this flow.

LES and hybrid LES-RANS simulations have performed better than the RANS models. Medium to high fidelity simulations have ranged from Detached- Eddy Simulations (DES) 
in [11], hybrid LES-RANS calculations [10, 13, 17], coarse LES reported in [9, 11, 12, 15], and LES performed on comparatively fine meshes [14, 16, 19]. Researchers in [11] simulated this flow with RANS, DES and LES closures, with the same grid used for the DES and LES calculations. The performance of a DES model with high viscosity at inlet was similar to its RANS counterpart, however a lower inlet viscosity model improved prediction performance. Typically, LES predictions were more accurate than DES. The hybrid LES-RANS models have been shown to perform better for this flow. Researchers in [10] used unsteady RANS close to the wall and switched to LES farther away. They applied turbulent velocity profiles from a DNS of a channel flow, and incorporated a forcing term at the interface between the LES and RANS regions to force the LES region to resolve fluctuations. The forcing improved flow field predictions in a plane downstream of the hill in the flow recovery region and outperformed LES that used only 1.7 million computational cells. An innovative two-layer scheme was used in [13], in which an LES was accompanied by an embedded inner region where parabolized turbulent boundary layer equations were solved to obtain the wall shear stress used by the LES as the wall boundary condition for the turbulent eddy viscosity. Their zonal scheme yielded satisfactory mean flow statistics in the separation region and downstream wake structures with $\approx 3.5$ million nodes, which was as effective as a conventional LES with 9.6 million nodes. In a more recent study in [17], the dynamic hybrid RANS-LES model developed in [20] provided reasonably accurate predictions, except for pressure distribution, the thickness of the recirculation layer, and turbulent stresses in a cross-stream plane downstream of the hill in the flow recovery region, which implied an erroneous wake structure.

LES on moderate to refined grids have yielded results superior to those obtained by hybrid LES-RANS closure models. The LES study in [12] used $\approx 5$ million computational cells, a dynamic $k$-equation sub-grid scale (SGS) model, an inflow that contained the experimental mean velocity profile in absence of the hill, and random white noise of $0.1 \%$ intensity to mimic the experimental approach flow. Its results compared reasonably well with experiments in the downstream separation region with a counter-rotating vortex pair. Later LESs on finer meshes have demonstrated the inadequacy of the grid resolution used in [12] and the consequent discrepancies in the outer flow. In a later work [14], implicit LES was performed on a refined mesh with $\approx 31$ million cells. The fact that the Reynolds number was lower by approximately $50 \%$ than for the experimental flow may affect the reliability of the comparisons. In [15], the LES was performed on a mesh with 15 million cells using the Smagorinsky SGS model. The study was intended to study the influence of the turbulent fluctuations prescribed at the inlet of the computational domain on the downstream separation. The inlet condition was obtained from a channel flow DNS at friction Reynolds number of 500. Significant discrepancies were noted in the thick and long separation bubble and the associated wall pressure distribution. More recently, results from two LESs were presented in [16] using $\approx 37$ million cells, with emphasis on wall-normal resolution, and $\approx 136$ million cells, with fine spanwise resolution. Both simulations used precursor simulations for emulating the turbulent inflow conditions in the experiments, and resulted in a detailed view of the separation, reattachment and flow recovery, including 
in-depth analysis of the flow topology and wake structure. Excellent agreement with the experiments was achieved for the pressure distribution and the kinetic energy contours in the midspan plane along the separation bubble thickness. Several intriguing aspects of the flow emerged, including the prediction of an earlier separation onset not observed in the experiment, a patch of high turbulence before the separation onset, and details of the separation dynamics. This work is, to the best of the authors' knowledge, the most detailed study of this flow configuration, and clearly illustrates the importance of highfidelity simulations.

However, in spite of earlier efforts, an important computational aspect remains unresolved. For high Reynolds number flows, precursor simulations for the generation of turbulent profiles at the simulation domain inflow significantly increase the computational cost. In [15] results of an LES with an experimental steady inlet velocity profile were compared with result of an LES with fluctuation fields from a low-Re precursor DNS superimposed on the experimental mean velocity profile in the absence of the hill. It was hypothesized that the influence of the approaching turbulence on the separation in the leeside of the hill may not be significant. However, it was also noted that this hypothesis ought to be verified by performing adequately resolved simulations at the Reynolds numbers of the experiments. On the other hand, superior results in DES simulations were obtained in [11] when a long inflow section was used, and it was concluded that 'the resolvable structures in the boundary layer are not negligible.' Authors in [21] also reported a significant influence of the velocity profiles prescribed at the inlet of the computational domain on the evolution of separation, reattachment and the vortical structures downstream of the hill, even at a lower Reynolds number $\operatorname{Re}_{H} \approx 6650$. In their simulations, two laminar profiles and a turbulent mean profile at different momentum thickness Reynolds numbers were prescribed at the inlet of the domain. The present work investigates this computational aspect in detail by simulating two adequately resolved LESs of this flow. For one of the LESs, an associated precursor simulation is performed to generate an inlet condition consistent with the experiment. For the second LES the experimental mean boundary layer profile in the absence of the hill is used as the inlet boundary condition. Both simulations are conducted on a grid with 45 million cells. The results show remarkable effects of the inflow boundary condition on the flow evolution both upstream and downstream of the hill. In conclusion, we find that the incoming turbulence has profound effects on the separational flow dynamics in the wake of the hill. However, upstream of the hill the formation of an internal layer triggered by a switch in pressure gradient results in a minimal effect of the approaching turbulence on the onset of separation.

At this point a brief discussion on the formation of internal layers is in order. Turbulent boundary layers can be subjected to sudden perturbations, such as changes of surface roughness, wall heating/cooling, pressure gradients, or changes in surface curvature. If the perturbations are strong enough, an inner region may form that evolves as an independent internal layer, while the outer flow maintains its properties upstream of the application of perturbation [22]. Formation of an internal layer is generally a consequence of excessive stress in the near-wall region that violates the equilibrium condition described by the law 
of the wall. Therefore, the logarithmic region is typically not obtained following a strong perturbation. Researchers in [23] and [24] found that the application of a strong favorable pressure gradient on a fully developed turbulent boundary layer causes the layer to be relaminarized. Formation of an inner region thus results in a quasi-laminar state of the near-wall flow. Once the pressure gradient is relaxed, the flow reverts to a turbulent state via laminar-turbulent retransition. While the flow properties change rapidly along a mean streamline inside the internal layer, they remain almost frozen in the outer flow. Thus, changes in the boundary-layer structure due to a strong pressure gradient are only experienced very close to the wall. A strong convex curvature also affects the near-wall flow. Authors in [25] showed that an active stress layer may form that is independent of the outer flow. In their experiments, a fully developed turbulent boundary layer was modified by a sudden convex wall that turned through $90^{\circ}$. A zero pressure gradient was maintained at the wall to distinguish the effect of wall curvature from the effect of the pressure gradient induced by it. The shear stress profiles for two experiments with different radii of curvature of the wall collapsed against the wall-normal distance normalized by the radius of curvature, rather than scaling w.r.t. the boundary layer thickness. The changes in the inner region took place at the onset of change in curvature; however, the changes in the outer region only took place later when the flow had turned through $13^{\circ}$.

Studies in [26, 27] of turbulent boundary layer flows over a two-dimensional hill and over a bi-convex airfoil are also highly relevant to the present work. The ratio of the boundary-layer thickness to the hill height $\delta / H \approx 0.4$ compared to 0.5 herein. An internal layer formed as the curvature of the hill switched from concave to convex; the internal layer was similar in its characteristics to the boundary layer developing over the airfoil, suggesting that the formation of the internal layer is independent of the outer flow. In the results presented herein, the internal layer is found to develop as the pressure gradient switches from adverse to favorable in the upstream side of the hill. The formation of the internal layer causes the near-wall profiles of the mean velocity and turbulent stresses to collapse for the LES cases with and without incoming turbulence at the inlet. However, the effects of different straining sources are difficult to separate, owing to the nonlinearity inherent in the flows. As is shown in this paper, the pressure gradient in the presence of the hill is due to inviscid processes. The pressure gradient ultimately dictates the flow dynamics until separation occurs via formation of the internal layer.

The paper is structured as follows. Section 2 describes the numerical experimental setup. This includes details on the LES SGS model, the computational grid used for both simulations, and the performance measures demonstrating the efficacy of the SGS modelling and the adequacy of the computational grid. In Sec. 3, the results from the two LESs, both time-averaged statistics, and results from the instantaneous flow are compared with the experimental results. The influence of the incoming turbulence on the separation onset and the downstream wake structure and recovery regions for the two simulations are examined in Sec. 4. Finally, results and their implications are discussed, and conclusions are drawn, in Sec. 5. 


\section{Numerical experiment setup}

The computational setup is for an axisymmetric hill geometry; the following analytical expression describes the dependence of the hill height on the hill radius $(r)$,

$$
\frac{y(r)}{H}=-\frac{1}{6.04844}\left[J_{0}(\Lambda) I_{0}\left(\Lambda \frac{r}{2 H}\right)-I_{0}(\Lambda) J_{0}\left(\Lambda \frac{r}{2 H}\right)\right]
$$

where, $\mathrm{H}$ is the height of the hill, $\Lambda=3.1926, J_{0}$ is the Bessel function of the first kind and $I_{0}$ is the modified Bessel function of the second kind. The radius of the hill at its base is $2 \mathrm{H}$. Authors in $[3,4,6]$ performed measurements of the flow around the model of a hill with height $H=0.078 \mathrm{~m}$, mounted on the floor of a $0.91 \mathrm{~m}$ wide, $0.25 \mathrm{~m}$ high and $7.62 \mathrm{~m}$ long test section of a low-speed wind tunnel. The maximum incoming velocity is $U_{0}=27.5$ $\mathrm{ms}^{-1}$. Based on the hill height, the Reynolds number $R e_{H}=\frac{U_{0} H}{v}=1.3 \times 10^{5}$, where $v$ is the kinematic viscosity of the fluid. The experiments were performed in flow with freestream turbulence intensity $\approx 0.1 \%$. In the absence of the model, a turbulent boundary layer is obtained with thickness $\delta \approx H / 2$ at the location of the top of the hill ( $x=0$ in our coordinate setup). The momentum-thickness Reynolds number of the boundary layer is $\operatorname{Re}_{\theta}=\frac{U_{0} \theta}{v} \approx 7300$, where $\theta$ is the momentum thickness.

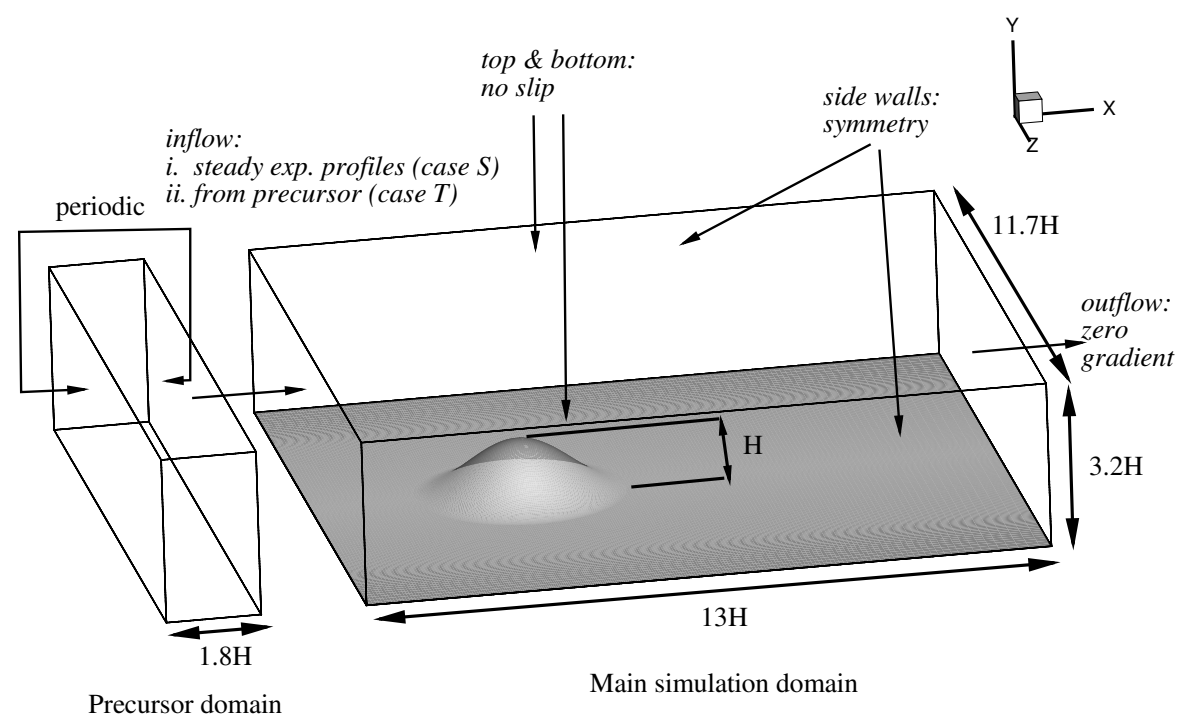

Fig. 1. Schematic of the setup for the numerical experiment.

This flow configuration was studied using large-eddy simulations (LES). The simulations were performed in a rectangular box with dimensions $13 \mathrm{H} \times 3.2 \mathrm{H} \times 11.7 \mathrm{H}$ in the streamwise $(x)$, wallnormal $(y)$ and spanwise $(z)$ directions, respectively (see Fig. 1 ). The objective of the simulations was to study the effect of the prescribed turbulence at the inflow of the simulation domain. To this end two simulations were performed. In one of the 
simulations, unsteady turbulent boundary layer profiles from a precursor turbulent channel flow simulation were applied to emulate the experimental turbulent condition in absence of the hill. The associated precursor calculation was performed in another rectangular box of length $1.8 H$, while the wallnormal and spanwise extents of the precursor simulation domain were the same as for the hill-simulation domain. We refer to this as case $T$. In the second simulation, a steady boundary-layer profile measured in the wind tunnel at the inflow of the hill simulation domain was prescribed. This simulation is referred to as case $S$.

\subsection{Large-eddy simulations \& Sub-grid scale modeling}

High-fidelity simulations are essential for capturing high-Reynolds number turbulent flow dynamics. For the flow under consideration, the cost of a direct numerical simulation (DNS) exceeds our computational resources, and hence, LES is the viable option pursued for the present calculations. For an incompressible flow, the continuity and momentum equations for the filtered velocity $\left(\tilde{u}_{i}\right)$ and pressure $(\tilde{p})$ fields are:

$$
\begin{gathered}
\partial_{i} \tilde{u}_{i}=0 \\
\partial_{t} \tilde{u}_{i}+\partial_{j}\left(\tilde{u_{i}} \tilde{u_{j}}\right)=-\partial_{i} \tilde{p}+\partial_{j}^{2} \tilde{u_{i}}+\partial_{j} \tilde{\tau_{i j}}
\end{gathered}
$$

where, $\tilde{\tau_{i j}}=\tilde{u_{i}} \tilde{u_{j}}-\widetilde{u_{i} u_{j}}$ is the sub-grid scale (SGS) stress tensor. We used the eddyviscosity dynamic Smagorinsky SGS model proposed in [28]. The model accounts for the SGS flow dynamics and its interaction with the resolved flow field.

In eddy viscosity SGS models, the deviatoric part of the SGS stress tensor is modeled as $\tilde{\tau_{i j}}-\frac{1}{3} \tilde{\tau_{k k}} \delta_{i j}=2 v_{S G S} \tilde{S_{i j}}$, where $v_{S G S}$ is the SGS eddy viscosity and $\tilde{S_{i j}}$ is the resolved rate-of-strain tensor. Usually, the isotropic SGS kinetic energy is absorbed in a modified expression for pressure. In the Smagorinsky model [29], $v_{S G S}$ is calculated as a function of the instantaneous magnitude of the strain-rate tensor $|\tilde{S}|=\sqrt{2\left|\tilde{S_{i j}}\right|^{2}}=\sqrt{2 \tilde{S_{i j}} \tilde{S_{j i}}}$.

$$
v_{S G S}=C_{D} \Delta^{2}|\tilde{S}|
$$

where, $\Delta$ is the filtering length scale which is proportional to the computational grid spacing. We calculated $\Delta=\left(\Delta V_{\text {cell }}\right)^{1 / 3}$ from the local cell volume $\left(\Delta V_{\text {cell }}\right)$. Unlike the Smagorinsky model, in the dynamic model $C_{D}$ is not a constant and is computed at every time step. An explicit test filter of size $2 \Delta$ was used to calculate the sub-test scale stresses. The difference between the sub-test scale stresses and the SGS stresses at sub-test filter scales is used to estimate the SGS dissipation provided by the eddy viscosity in a dynamic model [30], which is proportional to $C_{D}$. In our simulations, the calculation of $C_{D}$ is based on the method proposed in [28]. Instantaneously computed $C_{D}$ needs to be smoothed. Several smoothing techniques were attemped in preliminary coarse-grid simulations, such as under-relaxation in time [16] and local averaging over the faces of a computational cell [19]. For the reported simulations, $C_{D}$ is smoothed by averaging it locally over the faces of 
a computational cell, and then used to compute the eddy viscosity using Eq. 4. Alternative smoothing techniques do not significantly influence the results.

\subsection{Flow solver}

Equations 2 and 3 were solved using the open source CFD program OpenFOAM [31]. OpenFOAM solves the discretized equations in a collocated grid. The spatial discretization is performed with a finite volume method. Second order accurate Gauss linear interpolation for the fluxes from cell centers to cell faces was used for the calculation of the divergence and the Laplacian terms of the equations integrated over a cell volume. The time integration was performed using the Crank-Nicolson scheme. The overall accuracy of the solution is second order in both space and time. The 'pisoFoam' solver (i.e., the OpenFOAM implementation of the PISO algorithm for unsteady incompressible flows) was used. In each PISO time step, momentum prediction is performed once and the pressure correction loop is executed twice. Two iterations of pressure correction and flux updating to account for the grid nonorthogonality were performed within each pressure correction loop. The preconditioned conjugate gradient (PCG) and the preconditioned bi-conjugate gradient (PBiCGStab) algorithms were used for matrix inversions required to solve the pressure Poisson and momentum equations, respectively.

\subsection{Computational grid}

Fully resolved LESs were performed for both precursor and main simulations. While the hill geometry is axisymmetric, the computational domain is Cartesian. So, for the hill simulations, grid stretching was required in all directions. The number of grid points for the hill simulation was $501 \times 181 \times 501$ for the computational domain $13 \mathrm{H} \times 3.2 \mathrm{H} \times 11.7 \mathrm{H}$. For the precursor simulation associated with the case $T$, only the streamwise grid specification changes. 83 equally spaced grid points were used over a streamwise domain extent of $1.8 \mathrm{H}$. The total number of computational cells is 45 million for the hill simulation and 7.38 million for the precursor simulation. The grids used for these well-resolved LES were generated based on gradual grid refinement from earlier simulations on increasingly coarser grids reported in [19]. This flow was shown to be sensitive to grid resolution $[12,15,16,19]$. Hence, a detailed description of the computational mesh is given.

For the LES, grid spacings in all three directions in viscous wall unit defined by the friction velocity at the inlet of the hill simulation domain are shown in Fig. 2. The stretching ratio $(S R)$ of the grid spacings is also shown. In the left ordinate of Fig. 2(a), the scale is the wall-normal grid spacing in wall units $\Delta y+$, while the right ordinate shows the grid's stretching ratio $(S R)$. The height of the first cell adjacent to the wall corresponds to $\Delta y_{1}+\approx$ 0.81 . Six grid points are located within the viscous sublayer. Fourteen grid points resolve the flow in the buffer layer. $\Delta y+\approx 6.75$ at $y+\approx 100$ in the logarithmic layer. The $S R$ is $\approx 6 \%$ within wall-distance $y+\leq 50$ and decreases away from this location. The $y$-grid is adequately refined only for the lower wall. In the upper wall, wall-normal grid spacing is much coarser compared to the lower wall $\left(\Delta y_{1}+\approx 23.33\right)$, and is not shown here. 

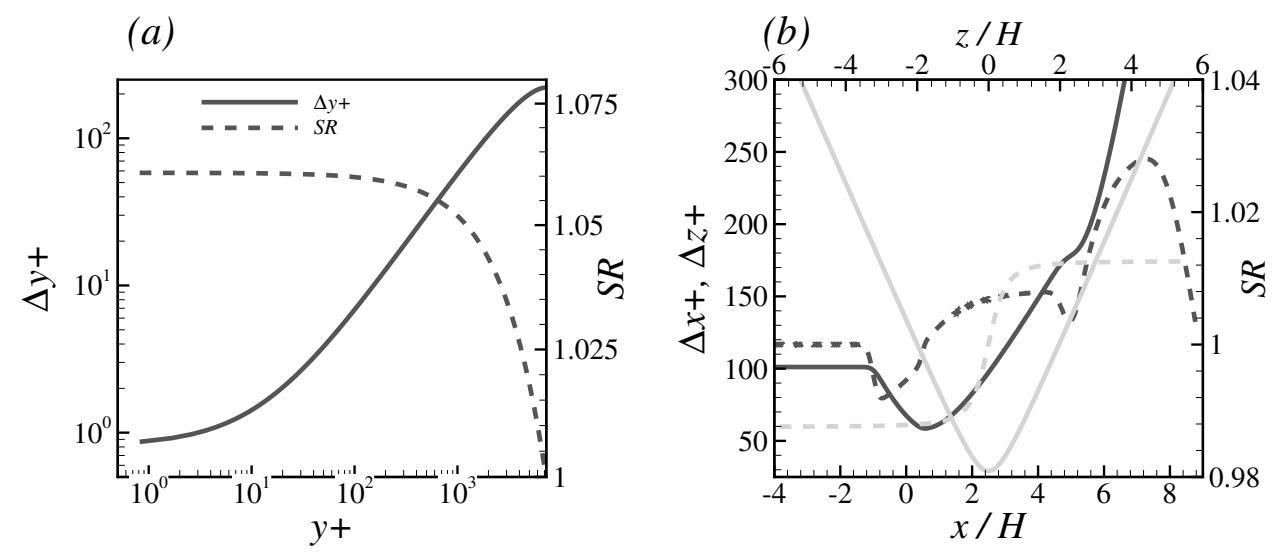

Fig. 2. Grid resolutions (solid lines) in viscous wall unit at the inlet of the main LES domain in the (a) wall-normal, $(b)$ streamwise and spanwise directions. Grid stretching ratio (dashed lines) is also shown for grids in all three directions. The dark and grey lines represent the corresponding curves for $x-$ and $z-$ grids, respectively. The $y-$ and $z-$ grids remained same for both precursor and main simulations.

Due to the high Reynolds number of the boundary-layer flow, the streamwise and spanwise grid resolutions shown in Fig. 2(b) were relatively coarse compared to recommended well-resolved LES resolutions [32]. In Fig. 2(b), the lower and upper abscissae represent the domain extents in the $x$ and $z$ directions, respectively. The left ordinate again shows the grid spacings in inlet-wall units for which the representative curves are the solid lines. The right ordinate shows the grid stretching ratio $(S R)$ for which the representative curves are the dashed lines. The curves for $x-$ and $z-$ grids are colored dark and light, respectively. The flow is expected to transition from an initial adverse pressure gradient (APG) in the concave region at the upstream foot of the hill to favorable pressure gradient (FPG) in the convex region in the upstream side of the hill to again APG in the downstream side of the hill where the separation takes place. The $x$-grid spacing is initially kept constant downstream of the inlet $(\Delta x+\approx 100$; this is the minimum streamwise resolution within the extents of the hill geometry). The grid spacing is then gradually refined downstream of the foot of the hill up to the location where separation is expected based on experiments and earlier simulations [19]. Downstream of the expected separation point, the $x$-grid spacing is gradually increased. In the lee side of the hill, post separation and reattachment, the flow is expected to recover to an equilibrium state. Beyond $x / H=5$, grid stretching is increased as the flow in that regime is not the subject of the present work; hence, a coarse resolution in that region may be deemed adequate. $z$-grid spacing is also gradually increased using the hyperbolic tangent function away from the center of the hill. With respect to the inlet-wall units, $29.16 \leq \Delta z+\leq 118.37$ within the spanwise extent of the hill geometry. Minimum $\Delta x+\approx 58.6$ in the lee side of the hill is almost twice the spanwise grid spacing in the midspan $(z=0)$ plane. $S R$ for the $x$-grid in the region of interest $(x / H \leq 5)$ is within 
1\%. SR for the $z$-grid is within $1.25 \%$ in the whole computational domain.
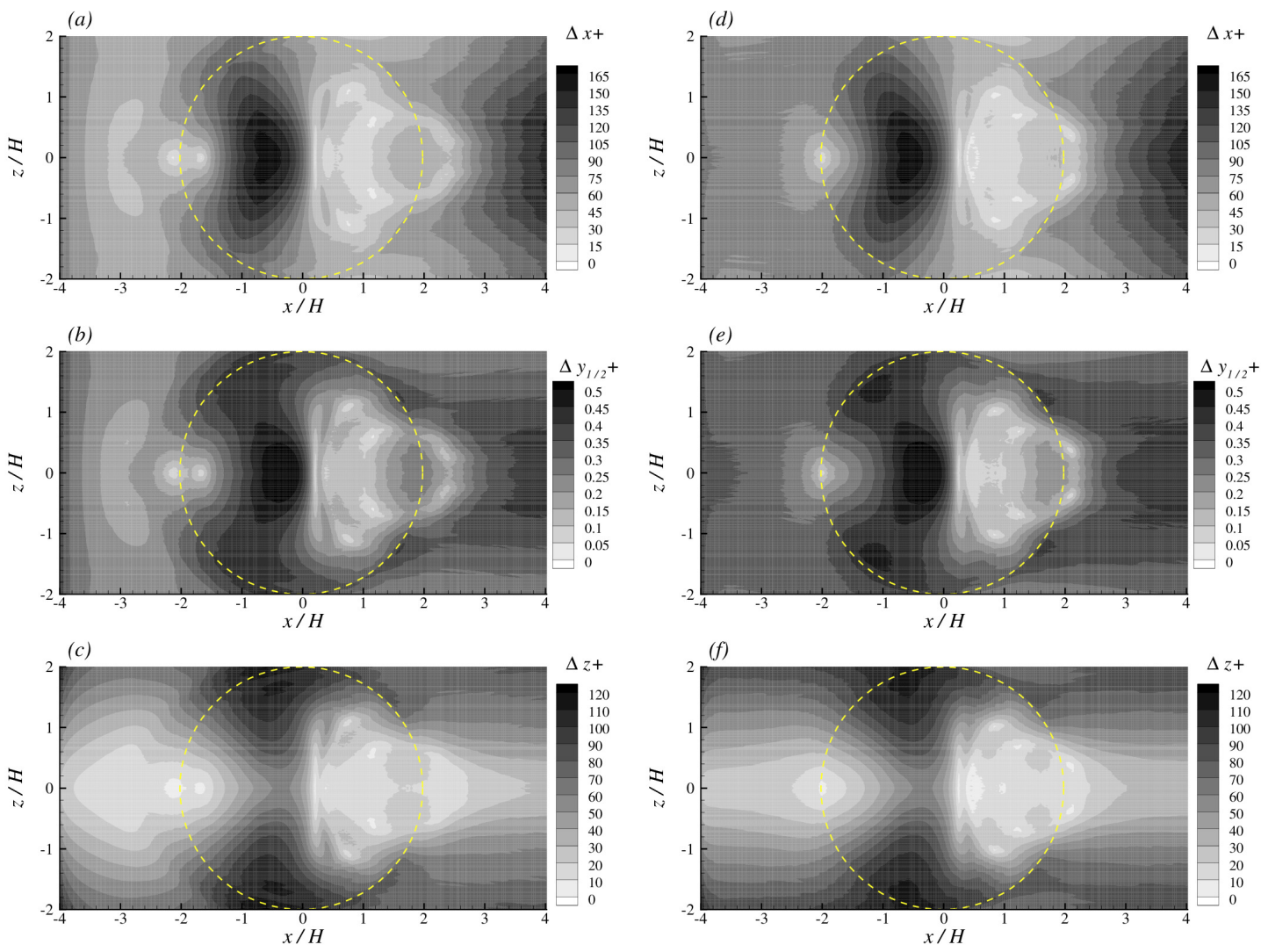

Fig. 3. Grid resolution in local viscous wall units from the LES: $(a)-(c)$ case $S$ and $(d)-(f)$ case $T$. $(a)$ and $(d)$ streamwise resolution $(\Delta x+) ;(b)$ and $(e)$ wall-normal resolution at the centroid of the first wall-adjacent layer of cells $\left(\Delta y_{1 / 2}+\right) ;(c)$ and $(f)$ spanwise resolution $(\Delta z+)$.

Grid resolutions in all three directions in local wall units obtained from the hill simulations are plotted in Fig. 3. Frames $(a)-(c)$ show the results from case $S$ and frames $(d)-(f)$ show the results from case $T$. In the plots, dashed circle mark the circumference of the outer edge of the hill. The contour lines are relatively smoother for case $S$, specifically in the region between the inlet and the foot of the hill because of the steady non-turbulent inflow condition. The contour characteristics are similar for both cases. Although $\Delta x+\approx 96$ at inlet for both simulations, it gradually decreases downstream. $\Delta x+<96$ in most of the simulation domain except in the upstream-side FPG region where the flow accelerates, resulting in higher friction velocity, and for $x / H>2.7$, where the grid spacing is gradually increased. Maximum value of $\Delta x+$ in the upstream-side FPG region is $\approx 169$. Figures $3(b)$ and $3(e)$ show the contours for $\Delta y+$ computed at the centroid of the first layer of cells adjacent to the wall for the cases $S$ and $T$, respectively. In the FPG region upstream of the hill, maximum $\Delta y_{1 / 2}+0.58$ for both simulations. Except right at the side walls, $\Delta y_{1 / 2}+\leq 0.58$. So, the $y$-grid is adequate for resolving the near-wall turbulence. As mentioned earlier, the $z$-grid 
is stretched in that direction. In the midspan plane $(z / H=0)$, peak value for $\Delta z+\approx 62$ is obtained in the FPG region in the upstream side of the hill for both cases. The value of $\Delta z+$ increases quickly in either side of the midspan plane. Maximum of $\Delta z+\approx 112$ is obtained at $|z / H| \approx 1.7$ within the circumferential extent of the hill, close to the outer periphery, in the flow acceleration region at $x / H \approx-0.38$. Except far away from the hill close to the side walls, $\Delta z+<125$. Beyond $|z / H|=2$, it increases gradually towards the side walls where $\Delta z+\approx 170$. Grid stretching was necessary to sufficiently resolve the region of the flow of significance, which is the thin separation region within the circumferential extent of the hill observed in the experiments [3]. Despite the similar aforementioned contour value characteristics, significant discrepancies are noticeable between the two cases, especially in the separation region downstream of $x / H=0.2$, indicating different flow dynamics close to the wall. In our experience, the lee-side separation dynamics is very sensitive to the spanwise grid resolution and a reasonable agreement with the experiment is only possible upon adequate refinement of the spanwise grid [19].

\subsection{Boundary conditions}

Boundary conditions used for the LES are shown in Fig. 1. Except for inflow and outflow, boundary conditions at the four walls of the precursor simulation are the same as for the LES of the hill simulation. No slip and impermeabilitiy conditions have been used for the velocity at the top and bottom walls. At the side walls, a symmetry condition has been applied for velocity, so that the spanwise velocity component, $w=0$. At the outflow of the domain for the hill simulations, a zero streamwise gradient condition was applied for all variables. As previously mentioned, cases $S$ and $T$ only differ in the specification of the inflow boundary condition. At the inflow plane of the domain for case $T$, the unsteady turbulent velocity field at a computational plane normal to the flow direction saved at each time step of the precursor simulation was applied. The associated precursor simulation is periodic in the streamwise direction for computing the fully developed turbulent boundary layer flow field. For case $S$ on the other hand, the mean velocity profile from the experiment [3] was prescribed at the inflow. A zero-gradient condition was applied for pressure at all six boundaries of both the precursor and hill simulations.

The grid spacing in the upper wall is not adequate for wall-resolved LES $\left(\Delta y_{1}+\approx 23.3\right)$; because the first wallnormal grid point is located in the buffer region, the wall function prescribed in [33] was used there. This single-formula profile for the velocity matches the experimental profiles very well even in the uncertain buffer layer. To compute the

corrected $u^{*}$ from the formula for $y+=f(u+)$, a tolerance level of $10^{-7}$ or a maximum of 100 iterations of the Newton-Raphson root finding solver, whichever is satisfied earlier, was chosen as the convergence criterion.

\subsection{Calculations \& Sampling}

When performing the calculations for case $T$, the precursor simulation is carried out first. Once statistical stationarity is reached, calculation of statistics and saving data for pre- 
scribing at the inflow of the hill simulation domain is initiated. For all LESs, including the precursor and hill simulations, the time step in wall units, $\Delta t+=\Delta t u *^{2} / v \approx 1.4$ w.r.t. the friction velocity $u *$ at inlet. As per recommendations in [34], even though it was based on direct simulation of channel flow, the time step used for the present calculations is just about small enough to temporally resolve turbulence adequately. The very fine meshes herein impose a severe requirement on computing time, and therefore, the chosen $\Delta t$ for the calculations made the simulations feasible within the limits of our computational resources. The chosen $\Delta t$ resulted in a maximum Courant-Friedrichs-Lewy $(C F L)$ number of $\approx 0.8$ for the precursor calculation. The maximum $C F L$ number varied between $\approx 2.5$ and $\approx 8$ at each timestep for both LESs, cases $S$ and $T$. Although these values are quite high, they were intermittently obtained in a few grid points near the maximum acceleration region upstream of the hill crest and in a very few grid points in the separated shear layer. Flow averaging and data extraction were initiated for the hill LES only after the initial flow transients were passed. Then flow statistics were sampled for a period of $\Delta t_{a v}=246.8 H / U_{0}$, which is sufficiently long to obtain smooth first and second order statistics.

\subsection{Efficacy of the SGS model}

The SGS model performance in a well-resolved LES is measured by computing the ratio of the mean SGS viscosity $\left\langle v_{S G S}\right\rangle$ and the molecular viscosity $v$. Hereafter, $\langle\zeta\rangle=$

$\frac{\int_{0}^{t_{a v}} \zeta(x, y, z, t) d t}{t_{a v}}$ represents the temporally averaged variable $\zeta$ unless otherwise specified. It is assumed that the LES is accurate for $\left\langle v_{S G S}\right\rangle / v$ of the order of 10 and below 100 [35]. The maximum of $\left\langle v_{S G S}\right\rangle / v \approx 5$ for the case $T$ is obtained in the outer regions of the domain far away from the hill $(x / H>7)$ close to the under-resolved side walls $(|z / H|>5)$ (because $v_{S G S} \propto \Delta^{2}$ in Eq. 4 and the grid spacing increases away from the hill in all three directions). However, for case $S$, the maximum of $\left\langle v_{S G S}\right\rangle / v \approx 4$ is obtained in the high velocity gradient region between the separated shear layer and the recirculating flow in the hill wake. This is reflected in the contour plot of $\left\langle v_{S G S}\right\rangle / v$ for the case $S$ in the midspan $(z=0)$ plane shown in the top frame of Fig. 4. In the bottom frame, the same contour plot is shown for case $T$ for comparison. The maximum of $\left\langle v_{S G S}\right\rangle / v$ in the lower wall region near the hill mounting is higher for case $S$, as is evident from Fig. 4. For case $T$ in this region, $\left\langle v_{S G S}\right\rangle / v<2.75$ everywhere. In both the top and bottom frames, the maximum in this plane is found in the lee-side of the hill after separation and before reattachment. The peak value is obtained in the high shear region due to the separated shear layer away from the wall right after separation.

Further assessment of the SGS model performance is sought. A total eddy viscosity may be calculated based on the resolved Reynolds stresses from the LES. Linear eddy viscosity hypothesis relates the total eddy viscosity $\left(v_{T}\right)$ to the resolved Reynolds stresses according to the following expression.

$$
2 v_{T}\left\langle S_{i j}\right\rangle=-\left\langle u_{i}^{\prime} u_{j}^{\prime}\right\rangle+\frac{2}{3} k \delta_{i j}
$$



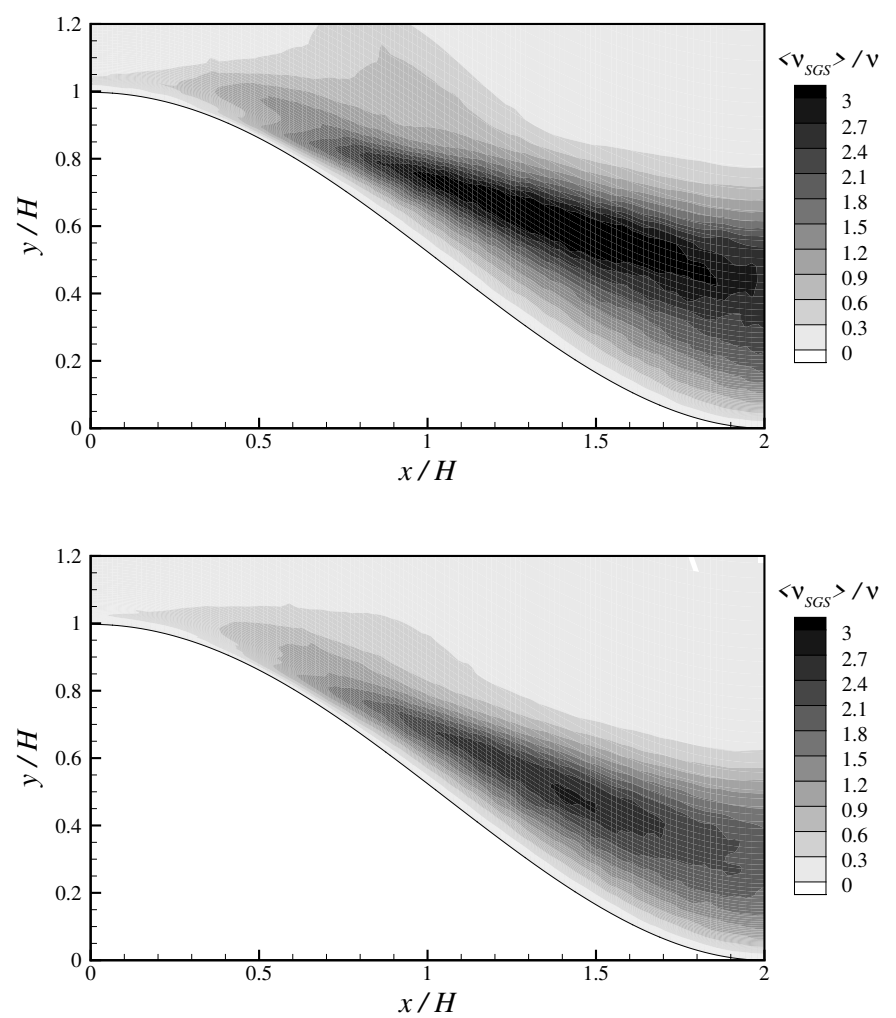

Fig. 4. Ratio of the mean SGS eddy viscosity to the molecular viscosity $\left(\left\langle v_{S G S}\right\rangle / v\right)$ in the midspan $(z=0)$ plane for cases $S$ (top frame) and $T$ (bottom frame).

where, $\left\langle S_{i j}\right\rangle$ is the mean rate of strain tensor. As the trace of the LHS matrix is zero, the second term on the RHS is added for equality. From this expression, $v_{T}$ may be extracted as,

$$
v_{T}=\frac{\left|-\left\langle u_{i}^{\prime} u_{j}^{\prime}\right\rangle+\frac{2}{3} k \delta_{i j}\right|}{\left|2\left\langle S_{i j}\right\rangle\right|}
$$

$v_{T}$ may be considered to be the total eddy viscosity obtained from an equivalent RANS computation of the same flow from which the same Reynolds stresses and mean rate of strain are obtained. The total eddy viscosity scaled by the molecular viscosity $\left(v_{T} / v\right)$ and the ratio of the SGS to the total eddy viscosities $\left(\left\langle v_{S G S}\right\rangle / v_{T}\right)$ for case $S$ are plotted in the midspan plane in Fig. 5(a) and 5(b), respectively. High values of $v_{T}$ are visible in the downstream separation region, where $v_{S G S}$ plotted in the top frame of Fig. 4 was also large. A very thin patch of very high $v_{T} / v$ (of the order of 1000's) is also obtained very close to the wall, where the local velocity changes sign between the recirculation layer and the separated shear layer which is not apparent from the plot. The maximum value is obtained in this patch. Expectedly, the maximum value of $v_{T}$ is more than 1000 times larger 

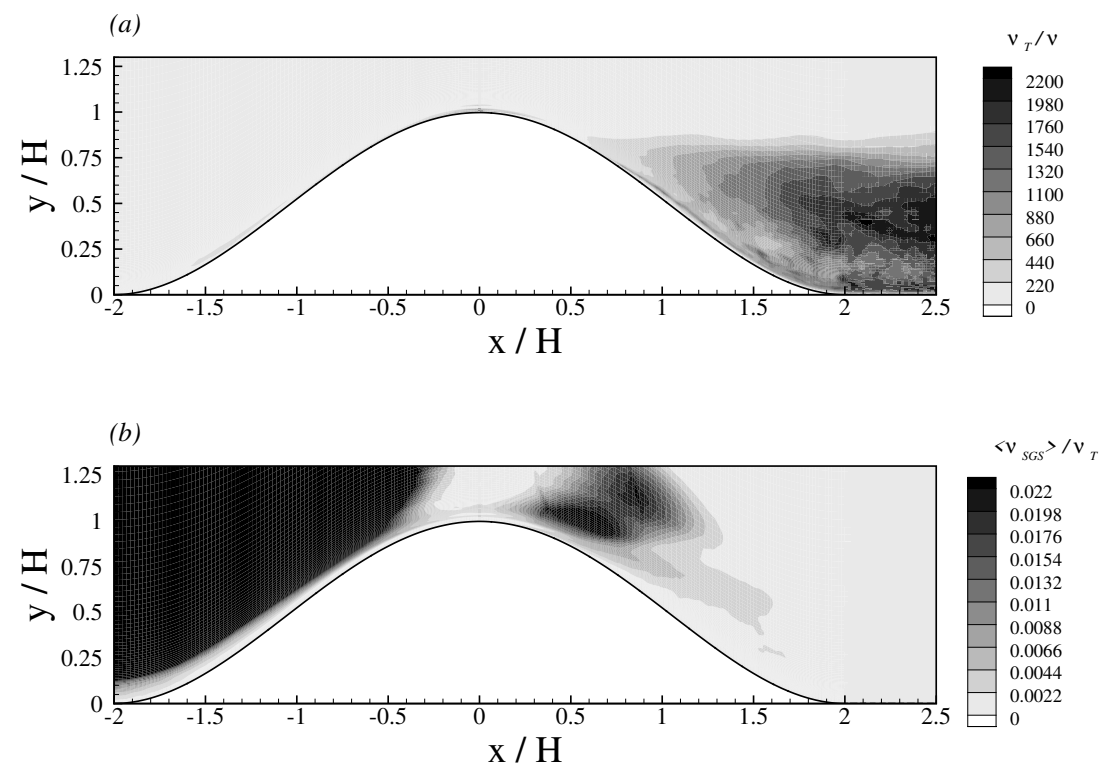

Fig. 5. Ratio of $(a)$ the total eddy viscosity to the molecular viscosity $\left(v_{T} / v\right)$ and $(b)$ the mean SGS eddy viscosity to the total eddy viscosity $\left(\left\langle v_{S G S}\right\rangle / v_{T}\right)$ in the midspan $(z=0)$ plane for case $S$.

than $v .\left\langle v_{S G S}\right\rangle / v_{T}$ quantifies the proportion of the SGS contribution to the total stresses (resolved plus modeled) obtained from the simulation. Maximum mean $v_{S G S}$ is only about $2.2 \%$ of the local $v_{T}$. Importantly, the ratio $\left\langle v_{S G S}\right\rangle / v_{T}$ is low in the high-flow-gradient and separation regions downstream of the hill, implying that most of the stress contributions to $v_{T}$ is due to the resolved component. High values were obtained either in the free stream away from the wall where grid resolution is coarser, and also in a small patch, also away from the wall, in the lee-side around the streamwise region where the mean separation is initiated.

Plots similar to those of Fig. 5 are shown for case $T$ in Fig. 6. Computed total eddy viscosity is lower for case $T$ compared to case $S$, as is evident from a comparison of the plots for the two cases. In the wake of the hill, a very thin patch is visible right next to the wall for case $T$, with peak values of $v_{T} / v$, similar to the case $S$. The SGS contributions to the total eddy viscosity are also lower for case $T$. Higher values of the ratio $\left\langle v_{S G S}\right\rangle / \nu_{T}$ are obtained only farther away from the wall in the upstream side flow in Fig. 6(b) compared to case $S$ in Fig. 5(b). Figures 4, 5 and 6 demonstrate the efficacy of the SGS model and the quality of the LESs presented herein. Turbulent stresses are due in almost their entirety to the resolved component, and the SGS contribution is negligible for both cases $S$ and $T$. 

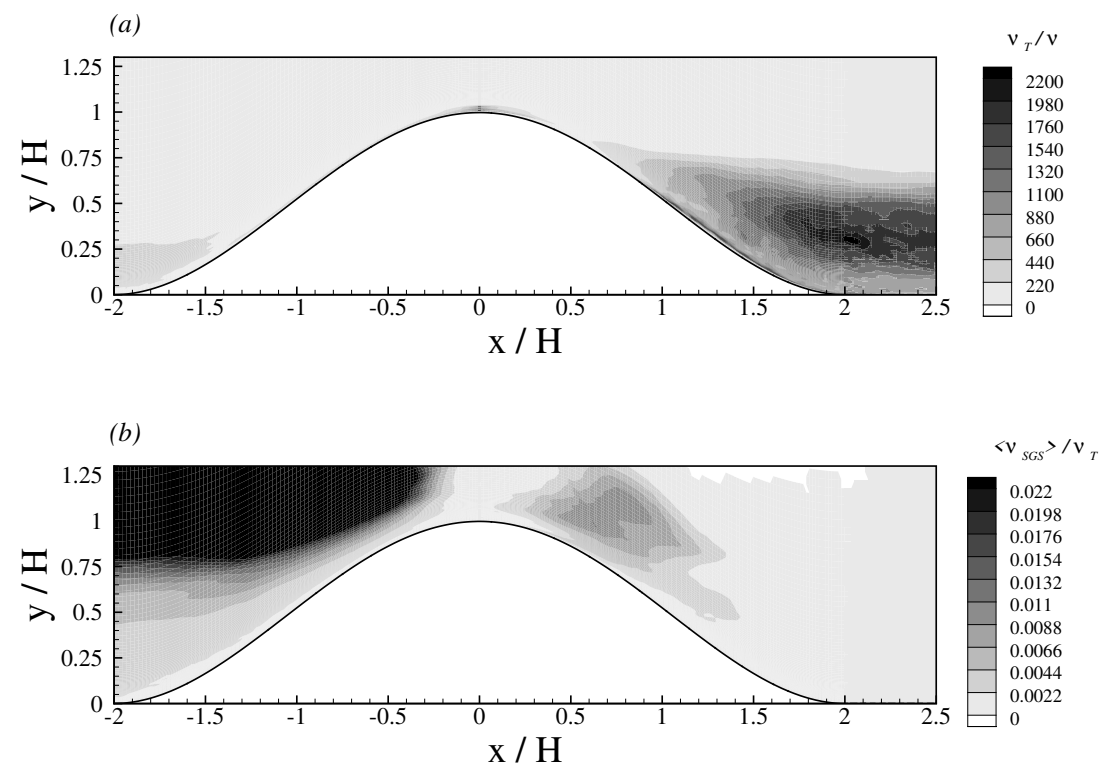

Fig. 6. Ratio of $(a)$ the total eddy viscosity to the molecular viscosity $\left(v_{T} / v\right)$ and $(b)$ the mean SGS eddy viscosity to the total eddy viscosity $\left(\left\langle v_{S G S}\right\rangle / v_{T}\right)$ in the midspan $(z=0)$ plane for case $T$.

\section{Comparison with experiments}

Based on LES simulations on coarser grids $(\approx 15$ million cells) using a turbulent boundary layer prescribed at the inflow from a precursor low-Reynolds number channel flow simulation, Author in [15] hypothesized that, for the hill-flow under consideration, the effects of the incoming turbulence on the flow separation are weak. However, no study showed that this is indeed the case either for well-resolved LES, or for high Reynolds number turbulent precursor simulations used to generate the inflow condition. In fact, simulations with three types of velocity profiles (both laminar and turbulent) prescribed at the inlet and reported in [21] demonstrated that, even at a lower Reynolds number, the inflow condition did influence the evolution of separation, reattachment and the vortical structures downstream of the hill. Simulation results presented in [19] for both fine and preliminary coarser grids also suggested that the properties of the turbulent boundary layer used at the inflow of the computational domain affect the flow. In the following subsections, we present results of precursor simulation for the LES of case $T$, LES results for cases $S$ and $T$, and extensive comparisons with experiments.

\subsection{Precursor LES corresponding to case $T$}

Precursor experiments were first performed $[3,6]$ to measure boundary-layer profile and turbulence properties in absence of the hill. A developed turbulent boundary layer with 
half hill-height thickness ( $\delta \approx H / 2 \approx 39 \mathrm{~mm}$ ) was obtained corresponding to $\operatorname{Re}_{\theta} \approx 7300$. To obtain an unsteady turbulent boundary layer matching an experimental profile at such a high Reynolds number at the inflow of the hill-simulation domain for case $T$, a forcing technique was required for the precursor simulation as in [36]. In this technique, the following expression is used to correct the streamwise velocity field at each time step so that the mean velocity matches the experimental profile, $U_{e}(y)$.

$$
u(x, y, z, t) \rightarrow u(x, y, z, t)-\langle u(y, z, t)\rangle_{x}+U_{e}(y)
$$

where $\langle u(y, z, t)\rangle_{x}$ is the streamwise averaged instantaneous $u$-velocity. As in a fully developed channel flow simulation, a constant body force term proportional to the difference between the instantaneous bulk flow rate and the mean target bulk flow rate is added to the RHS of the u-component momentum equation in Eq. 3 at each time step everywhere in the simulation domain. With these additional terms, the flexible simulation scheme can also match the variance of the $u$-fluctuations. However, this technique was not used to generate inflow conditions for the fully resolved simulations, since an extension of the streamwise domain would have been required to compensate for the adjustment of the incoming synthetic turbulence.
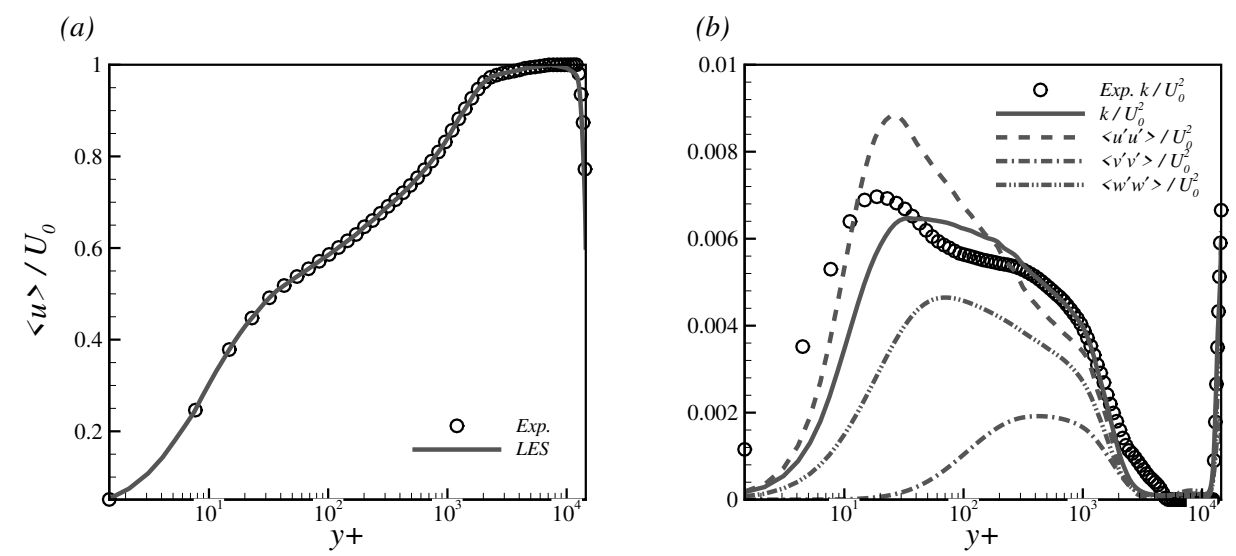

Fig. 7. Scaled wall-normal profiles of (a) time-averaged streamwise velocity $\left(\langle u\rangle / U_{0}\right)(b)$ turbulent kinetic energy and principal Reynolds stresses from the precursor simulation compared with the experimental results in [6].

In Fig. 7(a), temporally and spatially averaged mean streamwise velocity scaled by the reference velocity $U_{0}, \frac{\left\langle\left\langle\langle u(y)\rangle_{x}\right\rangle_{z}\right\rangle_{t}}{U_{0}}=\langle u\rangle / U_{0}$, is shown. Very good match with the experimental profile for the mean streamwise velocity is evident in Fig. 7(a). Scaled turbulent kinetic energy $\left(k / U_{0}^{2}\right)$ and diagonal components of the Reynolds stresses $\left(\left\langle u_{i} u_{i}\right\rangle / U_{0}^{2}\right)$ are plotted against wall-normal distance in wall units, $y+$ in Fig. 7(b). A discrepancy is noted in the near-wall peak in $k$ (the peak in streamwise Reynolds stress is at $y+\approx 26.5$ instead of $y+\approx 15$ ), which may be attributed to the lower resolution in $x$ and $z$-directions 
compared to resolution requirements for a well-refined LES using a second order method. However, the near-wall peak values of the r.m.s. fluctuations scaled by the friction velocity $u *$ are $2.68,1.245$ and 1.95 for the $u, v$ and $w$ components, which are in agreement with the experimental observations in [37], who reported the ratios 2.5, 1.3 and 1.9, respectively.
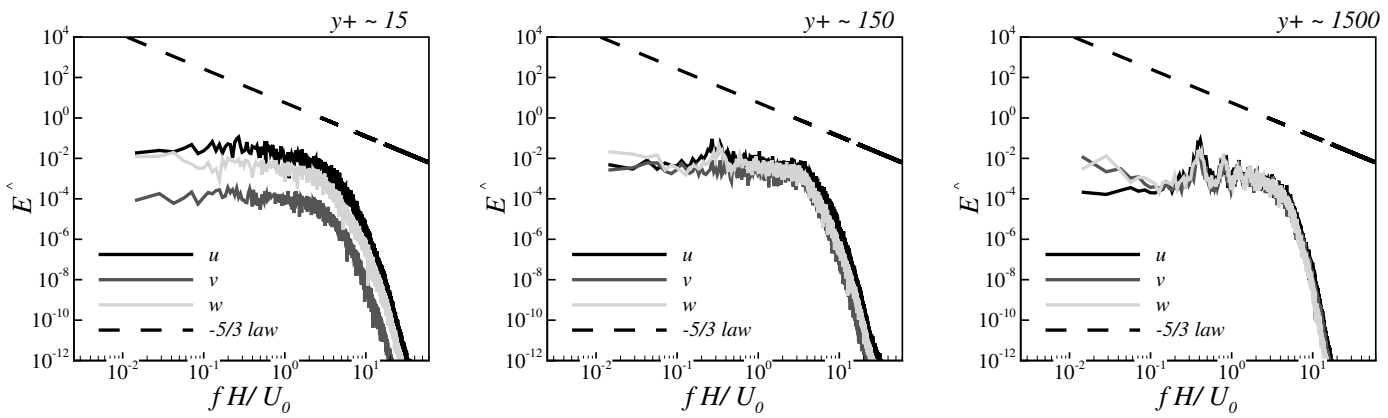

Fig. 8. Frequency spectra of the fluctuating velocity components at three wall-normal heights $y+\approx 15,150$ and 1500 calculated in wall unit.

Frequency spectra of the velocity fluctuations $(\hat{E})$ are shown in Fig. 8 at three wallnormal heights, $y+\approx 15,150$ and 1500 , which correspond to the buffer layer, logarithmic layer and outer region, respectively. The frequency $(f$ in $H z)$ is nondimensionalized by $H / U_{0}$. An approximate $-5 / 3$-range emerges for over a decade in frequency for all three components. At $y+\approx 150$ and 1500, plots for three components collapse on each other indicating the isotropic nature of small scale fluctuations. Because of the imposed streamwise periodicity, prominent peaks are obtained at $f H / U_{0} \approx 0.41$ and its higher harmonics, especially at $y+\approx 1500$ for all three components of velocity. However, close to the wall, at $y+\approx 15$, the effect of this periodicity is not evident in the frequency spectra. The streamwise periodicity does not affect the near-wall flow in the hill simulation results for case $T$. This has been verified by plotting the frequency spectra at different streamwise stations (see Fig. 26).

The two-point spanwise autocorrelation for the $u$-fluctuations,

$$
R_{u^{\prime} u^{\prime}}\left(\Delta z^{+}\right)=\frac{\left\langle u^{\prime}\left(z_{0}^{+}+\Delta z^{+}\right) u^{\prime}\left(z_{0}^{+}\right)\right\rangle_{t}}{\left\langle u^{\prime}\left(z_{0}^{+}\right)^{2}\right\rangle_{t}}
$$

is plotted in Fig. 9 at the same wall-normal heights as in Fig. 8. The flow in the current precursor LES is neither homogeneous nor periodic in the spanwise direction due to the imposed symmetry boundary condition. The grid is also stretched in that direction. Yet, for the precursor simulation, an approximate calculation of the spanwise autocorrelation function may be performed. The velocity components were first interpolated to a grid with equidistant spacing corresponding to average grid spacing in that direction. Close to the wall, vertical shear in the streamwise velocity induces streamwise elongated streaky flow structures. The size of those structures is reflected in Fig. 9. Very close to the wall in the 


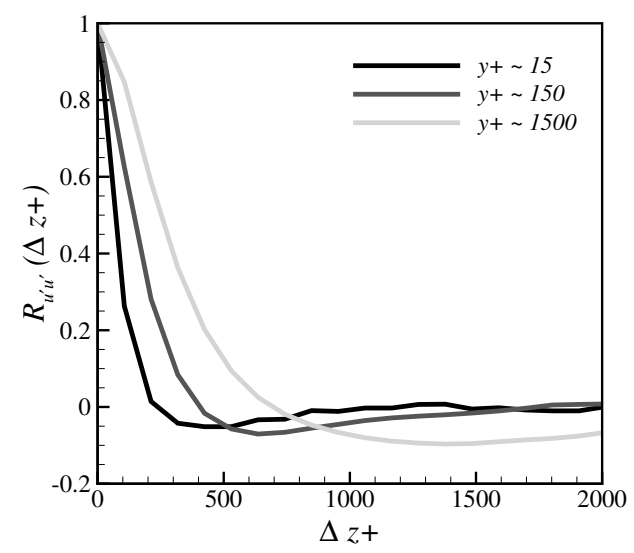

Fig. 9. Spanwise autocorrelation of the fluctuating streamwise velocity at three wall-normal heights $y+\approx 15,150$ and 1500 calculated in wall unit.

buffer region, the minimum correlation is obtained for $\Delta z+\approx 425$, which corresponds to the average streak width. Farther away from the wall, the structures get bigger and wider. At $y+\approx 150$, on average, the flow structures are of spanwise width $\Delta z+\approx 635$. For very high Reynolds number turbulent shear flows as in the present case, larger structures populate the region between the wall layer and the outer region. This plot shows that the near-wall structures are only nominally resolved in the spanwise direction. This likely results in the mismatch in the location of peak value of $k$ in Fig. 7.

\subsection{Results: Time-averaged flow}

\subsubsection{Pressure distribution}

The mean flow results from the LES simulations for cases $S$ and $T$ have been extensively validated against experimental results. In wall-bounded high-Re turbulent flows, the pressure coefficient $C_{p}=\frac{\langle p\rangle-p_{0}}{\frac{1}{2} \rho U_{0}^{2}}$, where $p_{0}$ is the reference pressure, is predicted with relative ease compared to other mean quantities such as Reynolds stresses. In the LESs for the flow under consideration, however, the $C_{p}$ distribution on the lower wall is found to be sensitive to grid resolution $[16,19]$ and inlet flow field, among other factors.

Figure 10 shows contours of the pressure coefficient, $C_{p}$ at the bottom surface of the simulation domain containing the hill, projected on the horizontal $(x, z)$-plane. The top frame shows the plot from the experiment, and the middle and bottom frames show the contour plots from cases $S$ and $T$, respectively. In all plots, the same contours as in the experimental result have been used. Additionally, $C_{p}$ along the midspan $(z=0)$ plane from the current LESs is compared with experiment and another high-resolution LES [16] from literature in Fig. 11. In the upstream side of the hill, good agreement with the experiment throughout the spanwise extent of the hill is evident from the contours in Fig. 10 

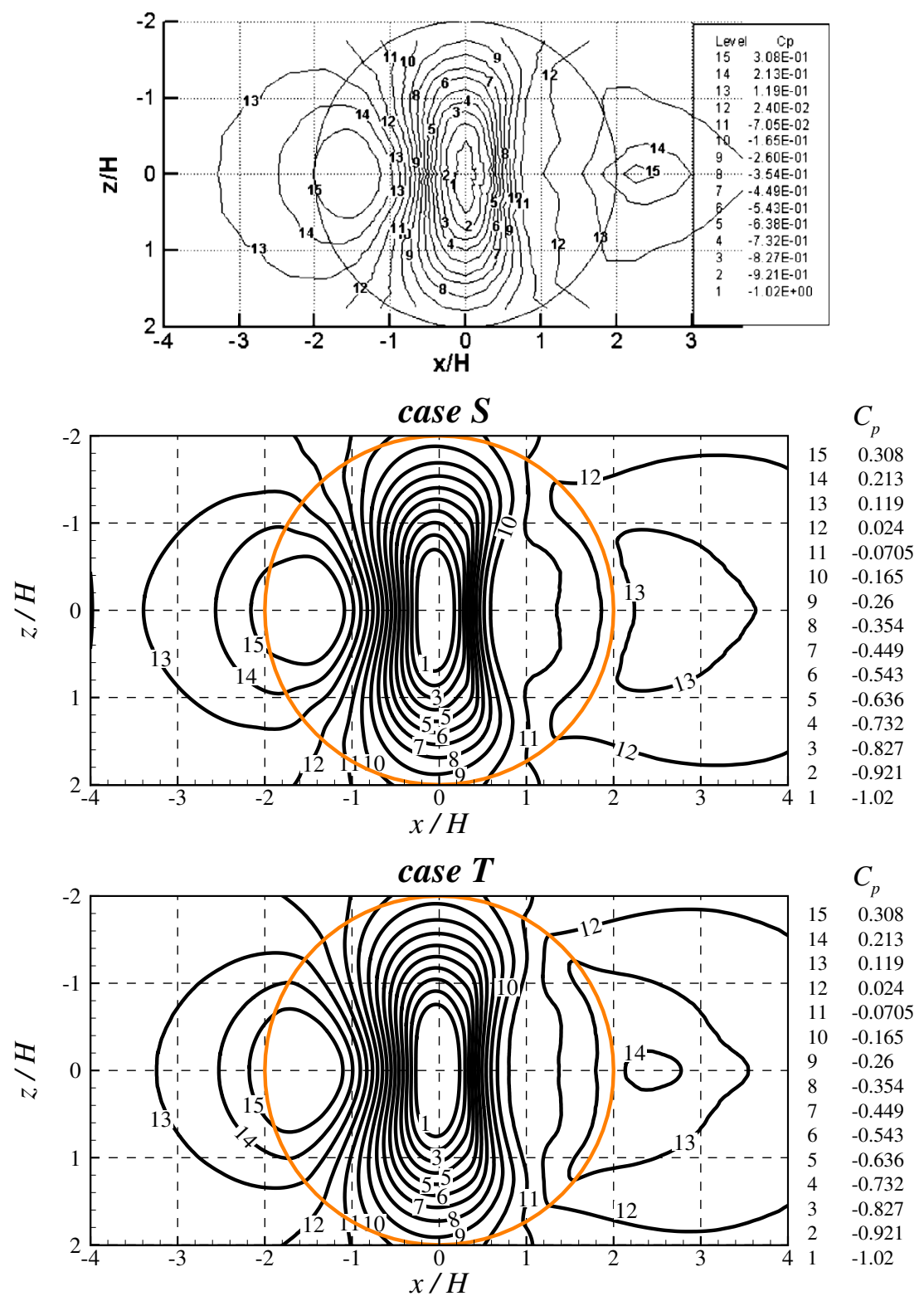

Fig. 10. Contours of the pressure coefficient $\left(C_{p}\right)$ plotted in the horizontal $(x, z)$-plane, from experiment $[3,38]$ (top frame), from case $S$ (middle frame), and from case $T$ (bottom frame). The circles in the frames show the outer circumference of the hill geometry.

for both LESs. Downstream of the inflow plane, the flow experiences an APG up to $x / H \approx-1.5$. Then onwards, $C_{p}$ drops rapidly indicating an FPG experienced by the flow over the upstream-side slope of the hill. Minimum pressure is attained just upstream of the crest of the hill, at $x / H \approx-0.038$ for case $S$ and at $\approx-0.023$ for case $T$ in Fig. 11 . Although the location of the minimum $C_{p}$ is consistent with the experiment and the com- 


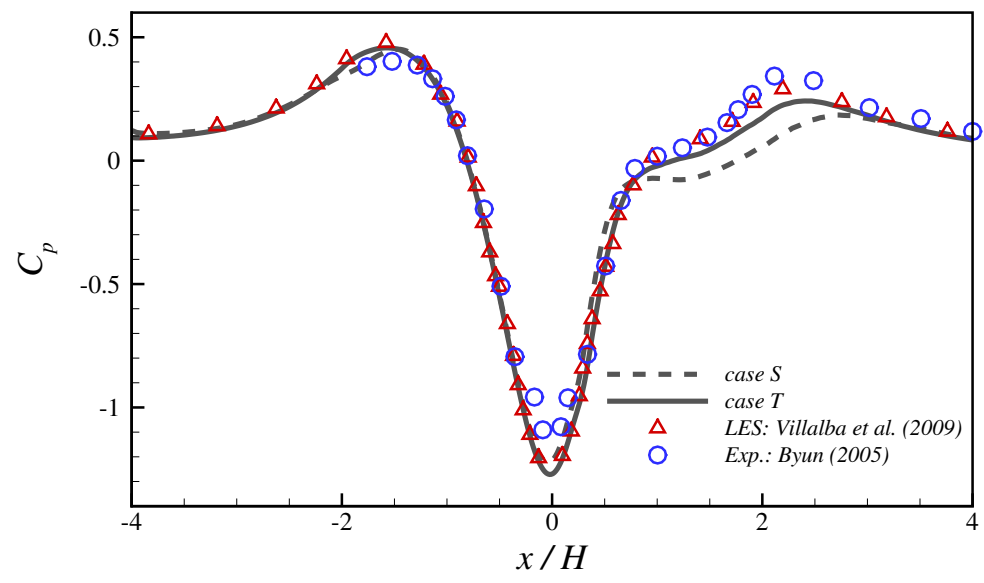

Fig. 11. Pressure coefficient $\left(C_{p}\right)$ along the $x$-axis in the midspan $(z=0)$ plane compared with experimental data $[3,38]$ and another high-resolution LES (the $L L$ simulation in [16]).

pared high-resolution LES, the minimum values of $C_{p}$ in current LESs are lower than the experimental minimum. The minimum $C_{p}$ in the present LESs is also slightly lower than the value obtained in [16], possibly due to lower resolution of the current simulations. The contours in the bottom frame of Fig. 10 for case $T$ match well with the experiment also downstream of the hill crest, where the flow initially experiences an APG followed by a FPG in the near wake beyond $x / H=2$. High values for $C_{p}$ are obtained in the experiments in the APG region at $x / H \approx 1.75-2$, where the flow reattachment takes place. Similar values of $C_{p}$ are also obtained for the case $T$ in that region, although w.r.t. the experiments, these contour characteristics are slightly dissimilar away from $z / H=0$. The peak value of $C_{p}$ in/ around the reattachment location $(x / H \approx 2$ in the experiment) is lower for case $T$ in both the figures. Low $C_{p}$ indicates a locally thicker recirculation layer close to the wall compared to the experiment. The overall comparison of case $T$ with the experiment is good.

Discrepancies are noted in the regions of flow separation and reattachment for case $S$ in both Figs. 10 and 11. The $C_{p}$ rises quickly beyond the location of minimum $C_{p}$ in the symmetry plane (see Fig. 11). In fact, in the region $0.25 \leq x / H \leq 0.75$, the curve for the case $S$ overshoots all other curves in Fig. 11 (curves for the case $T$ and the experiment collapse on each other in this region). After a quick rise in pressure, all the curves start flattening in Fig. 11 as the separation region thickens in the experiment for $x / H \geq 0.96$. Clearly, $C_{p}$ contours in Fig. 10 and the curve in Fig. 11 for case $S$ flatten the sooner than in the experiment. $C_{p}$ remains almost constant for the case $S$ in this streamwise region, visible in the noticeable inconsistency w.r.t. the experiment at $x / H \approx 1$ and beyond in Fig. 10, and the plateau $(0.8 \leq x / H \leq 1.3)$ in the curve for the case $S$ in Fig. 11. Such behaviour of the $C_{p}$ curve is indicative of a thicker flow recirculation zone for case $S$. On the other hand, the 
$C_{p}$ values for the case $T$ in Fig. 11 is slightly lower than the experimental value in most of the separation region $(x / H \geq 0.75)$.

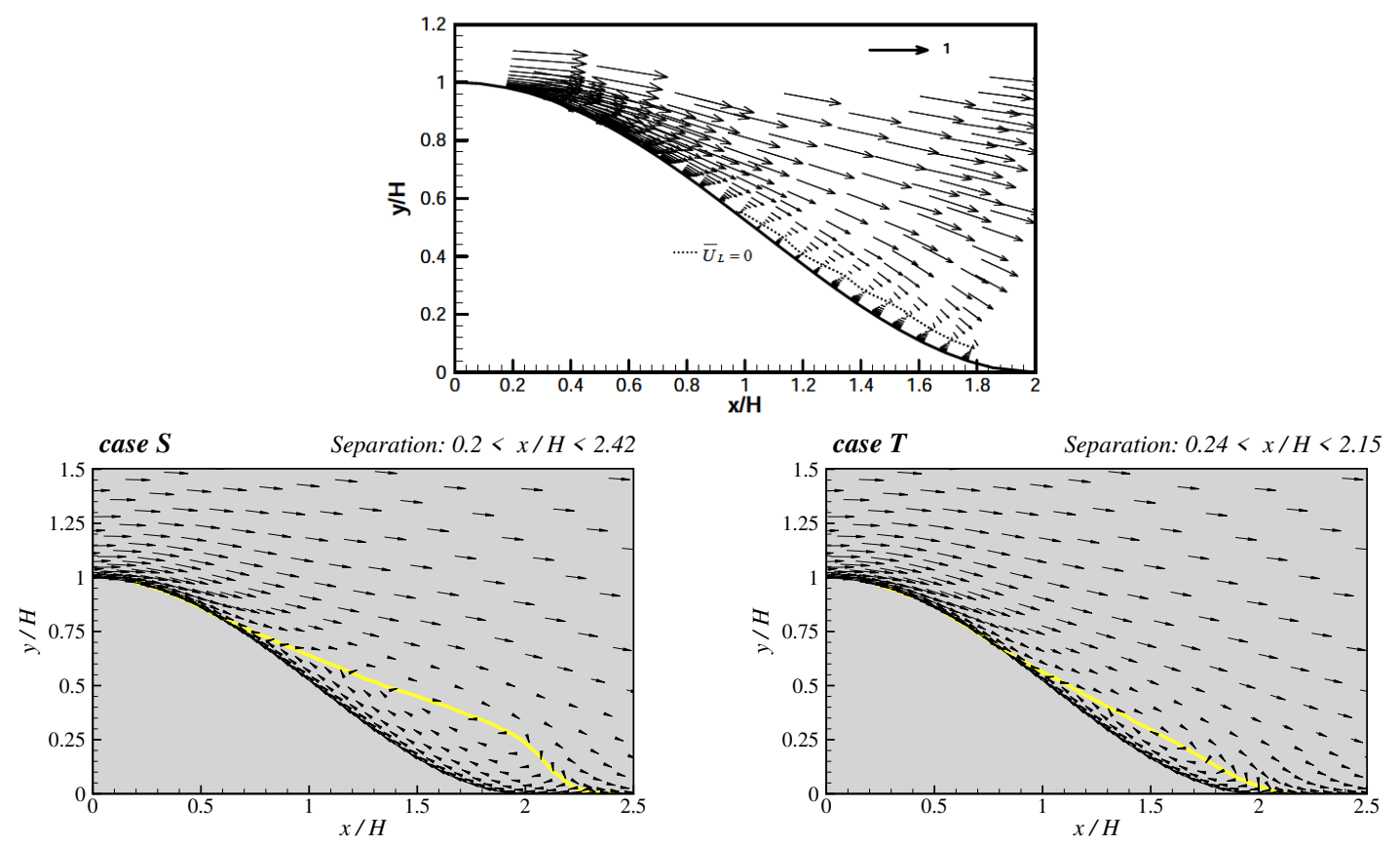

Fig. 12. Contour of the zero-mean streamwise velocity $\langle u\rangle=0$ and the inplane velocity vectors in the midspan $(z=0)$ plane in experiment [6] (top frame) and from current LESs (bottom frames).

Authors in $[13,16]$ found that correct prediction of $C_{p}$ on the downstream side of the hill surface is dependent on the successful capture of the flow in the very thin separation region. Additionally, although the $G V R$ group in [16] used $\approx 134.5$ million cells with higher resolution in $x$ - and $z$-directions, researchers in [16] attributed the more accurate prediction of $C_{p}$ by the $L L$-group to the better wall-normal grid resolution. The present work as well as the results in [19] corroborate both observations. The inflow boundary condition is important for the correct prediction of $C_{p}$. The inflow turbulent boundary condition indirectly affects the $C_{p}$ distribution. Flow acceleration in the upstream side of the hill, and consequent deceleration resulting in onset of separation is dictated by the inviscid processes due to the presence of the hill. Inspite of the difference in the inflow boundary condition, the separation is initiated around the same location for both cases. Downstream of separation, the incoming turbulence suppresses the recirculating flow. This results in an earlier reattachment and a better agreement with the experiment (more on this in Sec. 4). The $C_{p}$ distributions shown in Figs. 10 and 11 indicate that the lee-side separation dynamics is better captured in case $T$. 

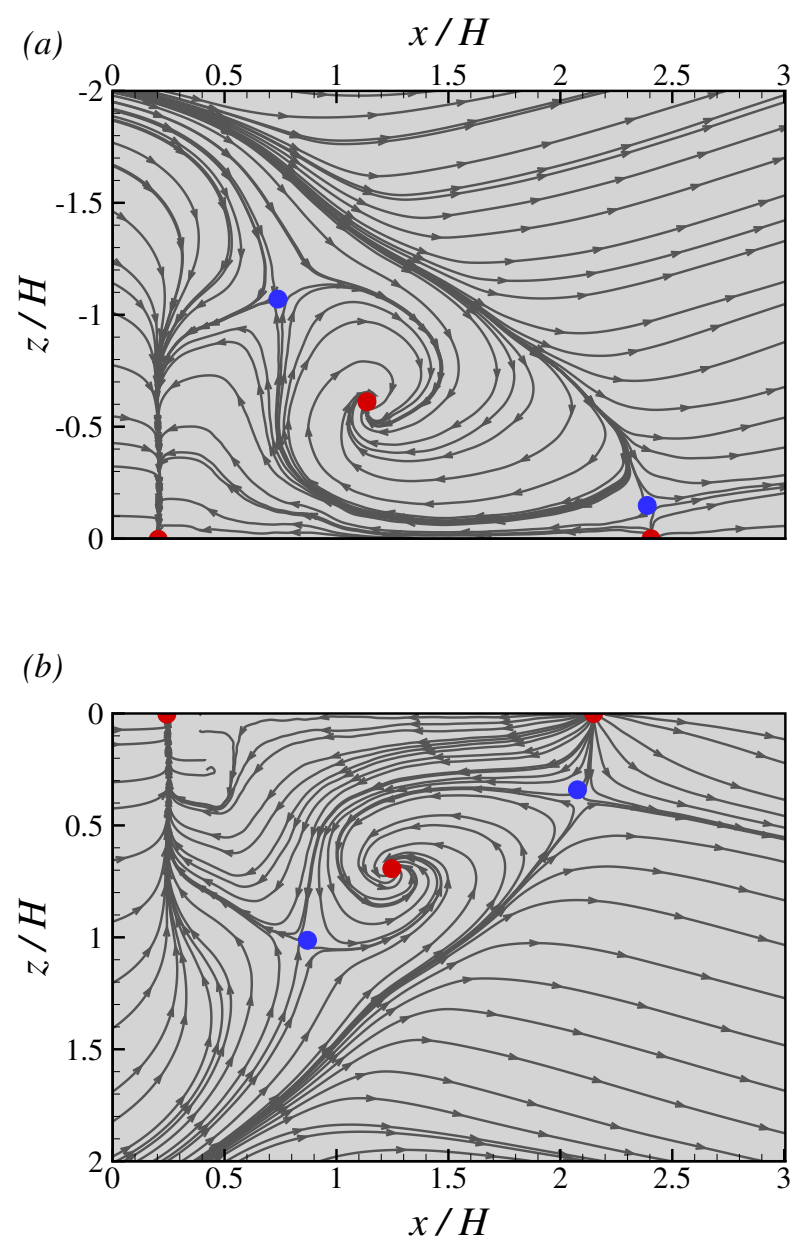

Fig. 13. Streamlines based on the mean skin-friction on the lower wall projected on to the $(x, z)$-plane depicting the near-wall flow topology from $(a)$ case $S$ and $(b)$ case $T$. The filled red (blue) circles indicate nodes (saddles).

\subsubsection{Lee-side separation \& reattachment}

The time-mean recirculation region in the midspan plane was reported from the experiment by showing the zero-mean streamwise velocity $(\langle u\rangle=0)$ contour. This figure from [6] is shown in the top frame of Fig. 12, which also includes the inplane velocity vectors. The bottom frames show the same results from the current LESs. In the LESs, separation in this plane is initiated at $x / H \approx 0.2$ for case $S$ and at $x / H \approx 0.24$ for case $T$. The onset of separation for both cases is earlier than in the experiment, according to which separation starts further downstream at $x / H=0.96$. Authors in [16] argued that the recirculation region right after the separation onset is very thin and could only be captured when the recirculation layer got thick enough to be captured by measurement instrumentation, and hence, the actual separation onset is earlier than experimentally reported. The reported separation 
onset in [16] is at $x / H \approx 0.3$. Using a finer near-wall mesh than used in [16] (maximum $\left.\Delta y_{1 / 2}<0.6\right)$, the current LESs predict an even earlier separation onset for the flow in this plane. Case $T$ also supports the finding in [16] that the initial recirculation layer is very thin in the region $x / H<0.9$. In fact, the separation initiation at $x / H \approx 0.24$ for case $T$ is followed by an immediate reattachment at $x / H \approx 0.39$ for the flow to separate again at $x / H \approx 0.55$. However, this phenomenon of separation, reattachment and reseparation is not reflected in the plots for $C_{p}$. That it is not observed in case $S$ is an artifact of the incoming turbulence and the grid stretching in the spanwise direction. Additionally, instantaneous separation is intermittent, and the recirculation layer is very thin in this region. The spanwise extent of this patch is also narrow $(|z / H| \leq 0.3)$, as shown in the plot for near-wall streamlines in Fig. 13. For case $T$, the recirculation layer thickness is mostly in good agreement with the experiment; this was validated by overlaying the figure in the top frame on the plot for case $T$ (not shown here). A mismatch arises in the delayed reattachment at $x / H \approx 2.15$ for case $T$ compared to the experiment and the LES results in [16], both of which report the mean reattachment at $x / H \approx 2$. For $x / H>2.1$, however, the mean reverse flow region is very thin. The delayed reattachment is reflected in the underpredicted $C_{p}$ for case $T$ in this plane in Fig. 11. As also suggested by the $C_{p}$ distribution in Figs. 10 and 11 , the recirculation layer for case $S$ is indeed very thick and long, with reattachment only at $x / H \approx 2.42$ compared to case $T$ in this plane. The difference between results for the two cases illustrates the importance of the inflow turbulence at high Reynolds number for correct prediction of separation for this flow.

A three-dimensional view of the mean separation dynamics in the wake of the hill is sought. In Fig. 13, streamlines of the mean skin-friction coefficient projected on the horizontal $(x, z)$-plane for cases $S$ and $T$ are shown in the top and bottom frames, respectively. The near-wall flow topology in only one-half of the map about the symmetry plane, $z / H=0$ is shown by depicting the region of interest, in which the separation and reattachment occur. For ease of comparison, opposing directions of the $z$-axis are shown for both cases. Additionally, the zero-shear-stress topological points [39] are also shown. There are four saddle points (streamlines both converge towards and diverge away from these topological points) and four nodes (streamlines either converge into or diverge away from these) for case $S$ in the whole plane, five of these singularities are visible in Fig. 13(a), namely, three nodes (the separation and reattachment points along the midspan plane, and the focal node marked by the red circles) and the two saddle points (blue circles). As was found in [39], the sum of the number of nodes is equal to the number of saddle points for this incompressible flow. Although the same topological features are obtained for the case $T$ in Fig. 13(b), there are significant differences in their positions between cases $T$ and $S$. The locations of the separation and reattachment nodes for both cases are shown in Fig. 12; the focal node for the case $S$ is closer to the symmetry plane, and the streamlines converging there seem to traverse a trajectory with a larger radius of curvature compared to case $T$. The presence of the focal nodes indicates an ejection of vortical structures from close to the wall. Therefore, for the case $T$, vortices ejected from the focal nodes are expected to be stronger compared to case $S$. Hence the discrepancy between the locations of the two saddle points 


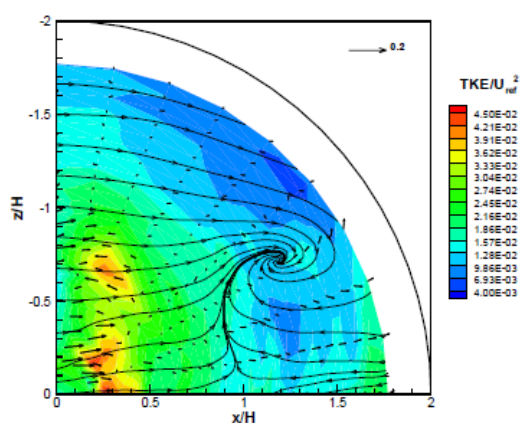

(a) $\mathrm{y}_{\mathrm{L} 0}{ }^{+}=11$

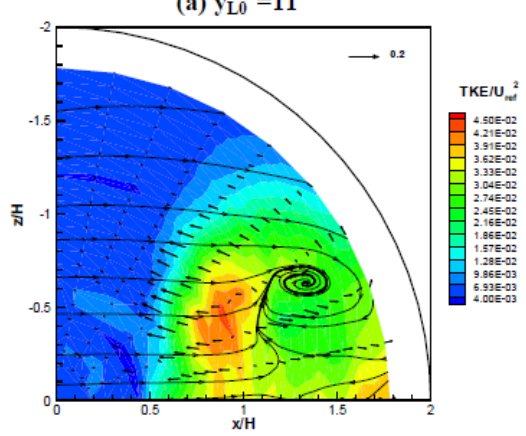

(c) $\mathrm{y}_{\mathbf{L} 0}{ }^{+}=\mathbf{2 0 0}$

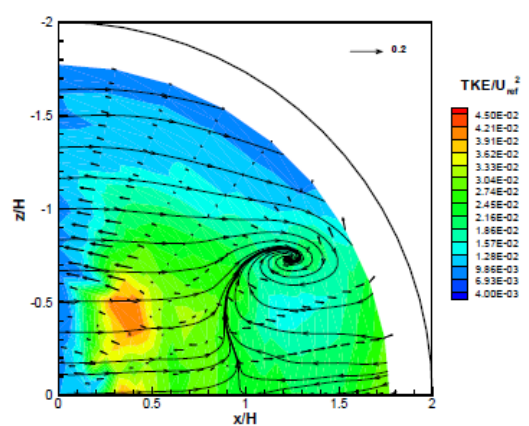

(b) $\mathrm{y}_{\mathrm{L} 0}{ }^{+}=41$

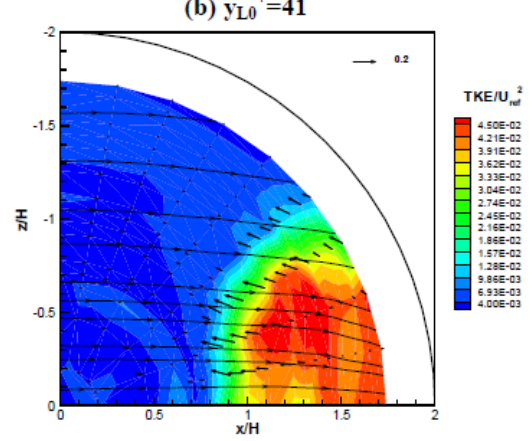

(d) $\mathrm{y}_{\mathbf{L} 0}{ }^{+}=\mathbf{5 7 8}$

Fig. 14. Streamlines based on the mean velocity field depicting the flow topology at different wall-parallel planes at selected heights w.r.t. the bottom wall, determined from the experiment [6].

visible in the shown halves of the plane for the two cases. Additionally, as was mentioned earlier, for case $T$, the initial separation is followed by reattachment and reseparation in a narrow spanwise patch $(|z / H|<0.3)$. This patch is visible right after the separation node in Fig. 13(b). In this small patch, the mean skin-friction streamlines converge/ diverge in an arbitrary pattern, and as per definition, several nodes and saddles can be identified, which are unphysical in our view. Apart from the discrepancy noted in this patch, and a slightly delayed reattachment, the topology map obtained for case $T$, that is, the locations of the focal nodes and saddle points, and the pattern of the streamlines is in good agreement with the $L L$ simulation in [16].

From an earlier oil-visualization experiment, authors in [3] reported a very complex flow pattern comprising three pairs of vortical structures close to the wall. An initial separation in the region $0.18 \leq x / H \leq 0.4$ was followed by a second larger separation accompanied by an adverse pressure gradient in the region $0.4 \leq x / H \leq 2$. In later LDV measurements in [6], a pair of near-wall vortical structures were found; the separation onset occurred much later at $x / H=0.96$. The presence of a pair of vortical structures close to the wall in our simulation of cases $S$ and $T$ is consistent with the results of [6], and contradicts the three-vortex-pair results reported in [3]. However, as shown in Figs. 12 and 13, the phenomenon of early separation followed by reattachment and re-separation in a narrow spanwise patch for the case $T$ is also consistent with the oil flow visualizations in 
[3]. Although the onset of the first and second separation regions for the case $T$ are similar to those reported in [3], a re-separation inbetween was not observed in the experiment. As mentioned earlier, it is likely that the reattachment after the initial separation for this case is due to grid stretching in the spanwise direction.

The mean separational flow topology farther away from the wall is presented from the experiment [6] in Fig. 14, and from cases $S$ and $T$ in Fig. 15 in frames $(a)-(d)$ and $(e)-(h)$, respectively, by plotting the mean streamlines constructed from the in-plane velocity vectors in chosen wall-parallel planes at different heights from the bottom wall projected onto the $(x, z)$-plane. The heights of these planes w.r.t. the viscous wall unit at the inlet of the simulation domain are indicated in the figures. Scaled turbulent kinetic energy contours $\left(k / U_{0}^{2}\right.$, henceforth denoted by $\left.T K E\right)$ with the same contour values as in Fig. 14, are also shown in these plots. In both cases $S$ and $T$, close to the wall, at $y+\approx 11$, mean streamlines initially curve inward towards the symmetry plane. The separation line along $x / H \approx 0.2$ (0.24) for the case $S(T)$ observed in the top (bottom) frame of Fig. 13 is only seen farther downstream at $x / H \approx 0.6(0.8)$ at the midspan plane that curls into the focal node, indicating, at onset, that the recirculation zone is very thin. In the experiment, the separation line is obtained at $x / H \approx 0.96$ at this wallnormal height also, followed by the streamlines curving into the focus. Also, in Fig. 15, the separation line is initiated at a saddle point in the midspan plane, which is separation node close to the wall shown in Fig. 13. There are three nodes and three saddle points for both cases in Fig. 15(a) and 15(e). The size and shape of the vortex emanating from the focal node is in good agreement with the case $T$, while significant discrepancies are apparent for the case $S$. The location of the focus in the current LESs is in good agreement with the experiment. Good agreement is also evident for the $T K E$ contours, especially for the high values. A patch of high $T K E$ is experimentally obtained at $0.3 \leq x / H \leq 0.4$ at $y+\approx 11$ in Fig. 14. The same patch of high $T K E$ can be seen for both LES cases around the same location. This patch is likely representative of the separated shear layer. This implies, in the experiments, that the separation onset very close to the wall is indeed earlier than $x / H \approx 0.96$, but was perhaps not captured owing to the limited resolution of the measurement instrumentation. This is discussed in more detail later.

Similar trends for the streamlines are seen farther away from the wall at $y+\approx 40$. The locations of the focus at all wallnormal heights from the LES case $T$ are in good agreement with the experiment. However, the initiation of separation is different from the experiment in both LES cases, i.e., it is farther upstream than in the experiment. In fact, at $y+\approx 200$, in the range $1.2 \leq x / H \leq 2$, streamlines traverse past the location of the separation lines at lower wallnormal heights and then curve outward and upstream to merge into the focal node in the experiment (Fig. 14). This trend is not visible for the LES cases presented herein, for which, the streamlines at this wallnormal height follow those at $y+\approx 41$ and 11. This indicates a stronger APG close to the midspan plane away from the wall in the LESs than in the experiment. Consequently, the recirculation region is thicker in the LESs compared to the experiment. This is evident from the comparison of the streamlines plotted at $y+\approx 578$. While for the experimental result in Fig. 14 no separation is obtained at this 

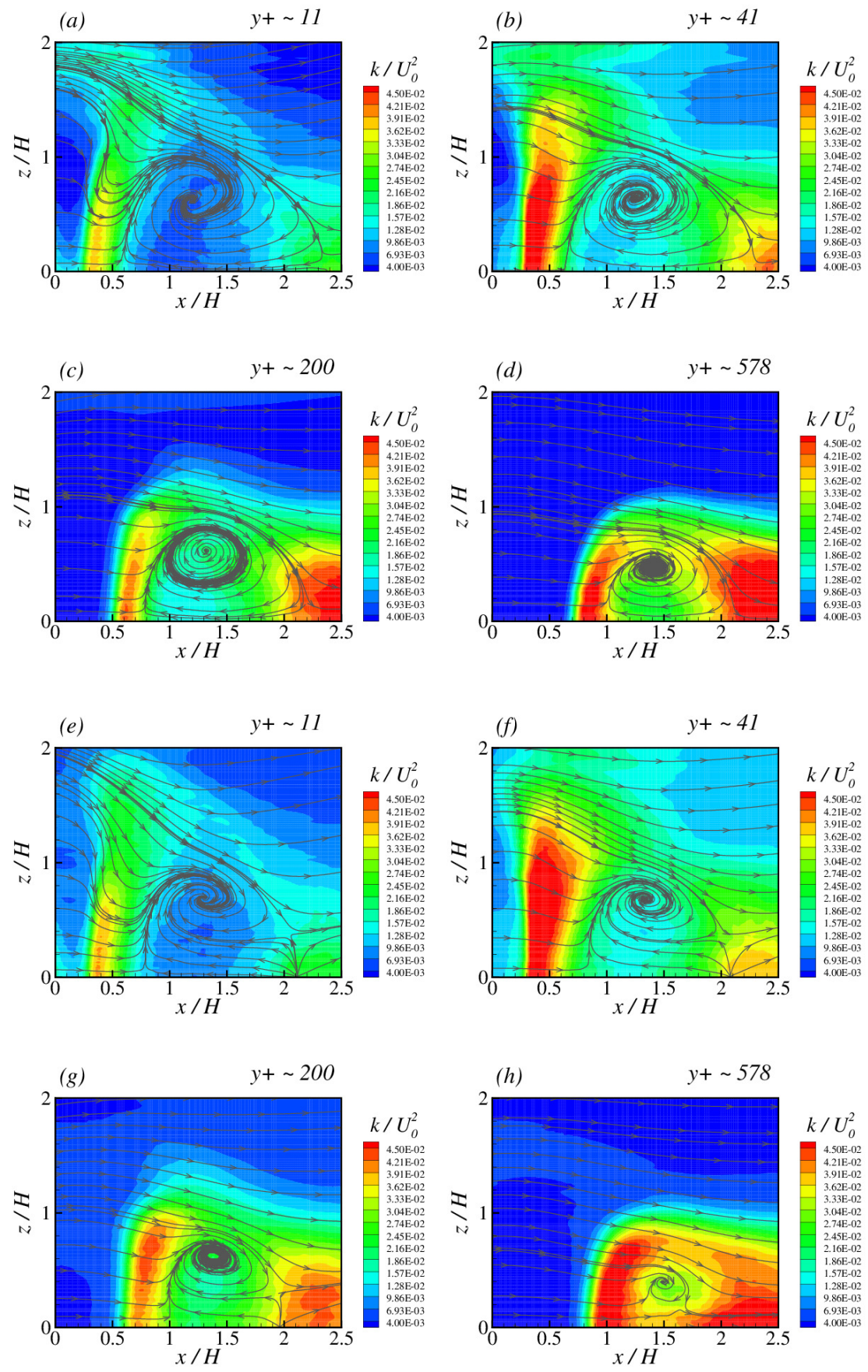

Fig. 15. Streamlines from in-plane mean velocity fields at wall-parallel planes at selected heights w.r.t. the bottom wall projected onto the $(x, z)$-plane obtained from the current LES: $(a)-(d)$ case $S$ and $(e)-(h)$ case $T$. The wall-normal heights of the selected planes are shown in inflow wall unit. 

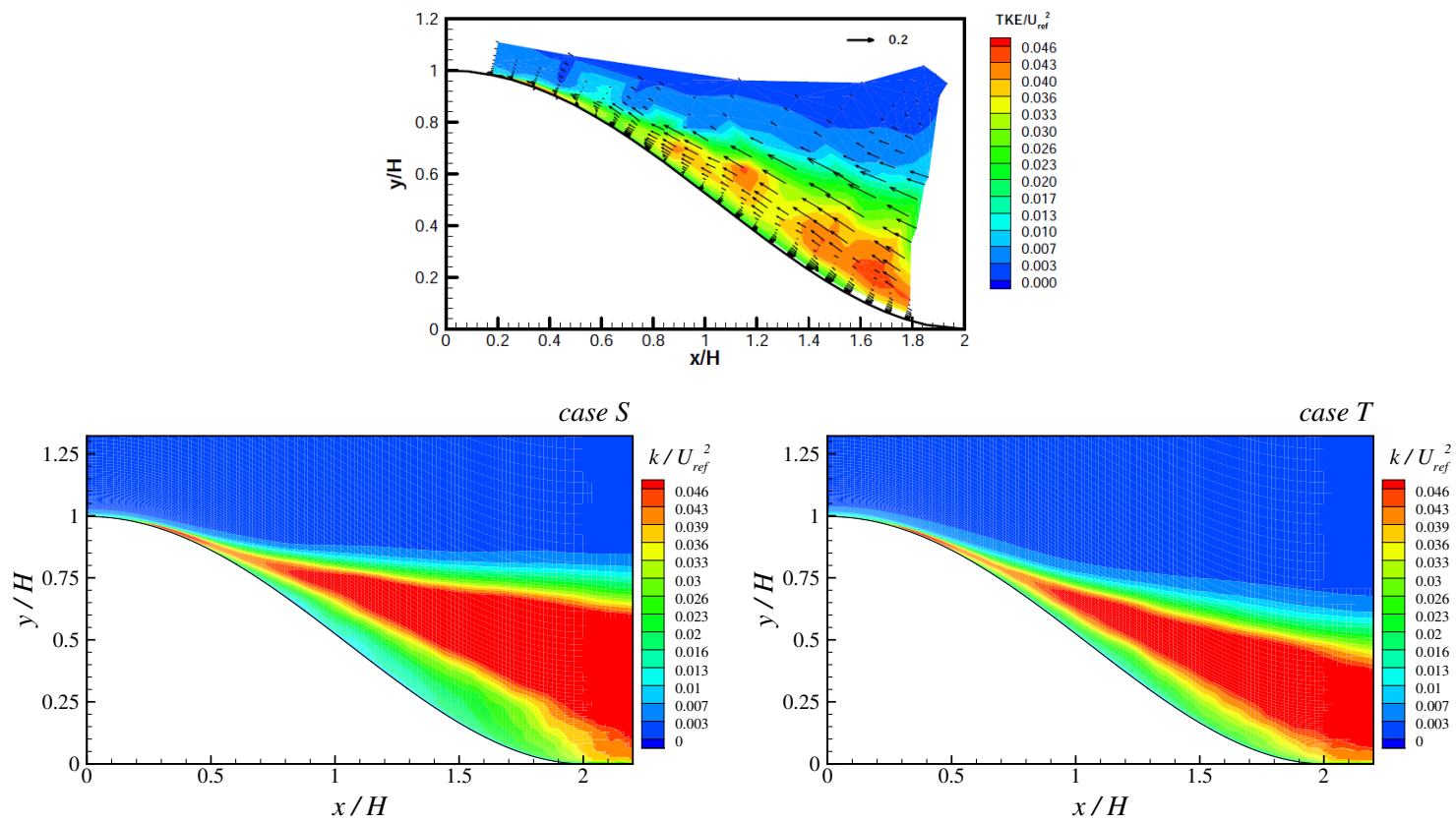

Fig. 16. Contours of the turbulent kinetic energy scaled by the reference velocity $\left(k / U_{0}^{2}\right)$ in the midspan $(z=0)$ plane from the experiment [6] (top frame), and from current LES: case $S$ (bottom left) and case $T$ (bottom right).

height, a mild focal node is visible for the case $T$ in Fig. 15(h) away from the midspan plane. On the other hand, for case $S$, a strong focal node is obtained at this height with a separated flow in the region $1 \leq x / H \leq 2.1$. The mean separation dynamics for the case $T$ is in good agreement with the experiment at all wallnormal heights. The $T K E$ contours are in accordance with the experimental results. High $T K E$ values are associated with the near-wall onset of separation at $y+\approx 11$ and 41 . Therefore, the pattern of the TKE contours depicts the 3-D orientation of the separated shear layer at different wallnormal heights. Patches with high $T K E$ are correctly predicted for both cases at all wallnormal heights, especially for case $T$. For example, at $y+\approx 200$, a patch of high $T K E$ is seen in the experiment in the region, $x / H \approx 1$ and $0.2 \leq|z / H| \leq 0.5$ in Fig. 14. This patch is, qualitatively, correctly predicted for case $T$ in Fig. 15(g). For case $S$, high $T K E$ obtained close to the midspan plane, decays farther away from this plane. Interestingly, $T K E$ values are low in and around the vortex being ejected from the focal node and increase farther away from it, implying the turbulence in these regions is associated with the local straining of the flow.

Contours of $T K E$ are plotted in the midspan $(z=0)$ plane in the top frame of Fig. 16 from the experiment [6], and in the bottom left and right frames of Fig. 16 from the LES cases $S$ and $T$, respectively. A thin region of high turbulence activity is obtained very close to the wall from $x / H \approx 0.2$ onward in both the experiment and the current LESs. In 
the bottom frames of Fig. 16, this patch extends up to the point of reattachment beyond $x / H \approx 2$. In case $S$, separation is initiated at $x / H \approx 0.2$, and in case $T$ at $x / H \approx 0.24$. In the LES, this patch is obtained around the onset of separation. It is possible that after separation, the high velocity gradient due to the separated shear layer can significantly increase the turbulence production. Therefore, this highly turbulent region belongs in the separated shear layer. Locally high turbulent contour values in the experiment depict the separated shear layer moving down the hill. The LESs confirm that the separation initiation in this plane was farther upstream than the reported separation onset in the experiments $[5,6]$. Right after separation, the very thin recirculation region could not be captured in the experiment. A similar observation of a highly turbulent region near the hill surface after separation onset was based on the high-resolution simulations in [16]. Although, downstream of $x / H \approx 0.7$, values of local peak values of $T K E$ are higher in the current LESs (this is not clearly visible from the contour plot owing to the same contour levels as in the plot based on experiment), the wallnormal locations of the high contour values for case $T$ are in agreement with the experiment. Therefore, it may be inferred that, qualitatively, the downstream evolution of the separated shear layer was correctly captured in the LES case $T$. On the other hand, as depicted by the contour plot for the case $S$, the separated shear layer is thicker, and so is the recirculation region beneath it (see Fig. 12). As seen from the flow topology maps of Figs. 13 and 15, and is confirmed by Fig. 16, the recirculation region right after separation is very thin, and therefore, difficult to capture correctly.
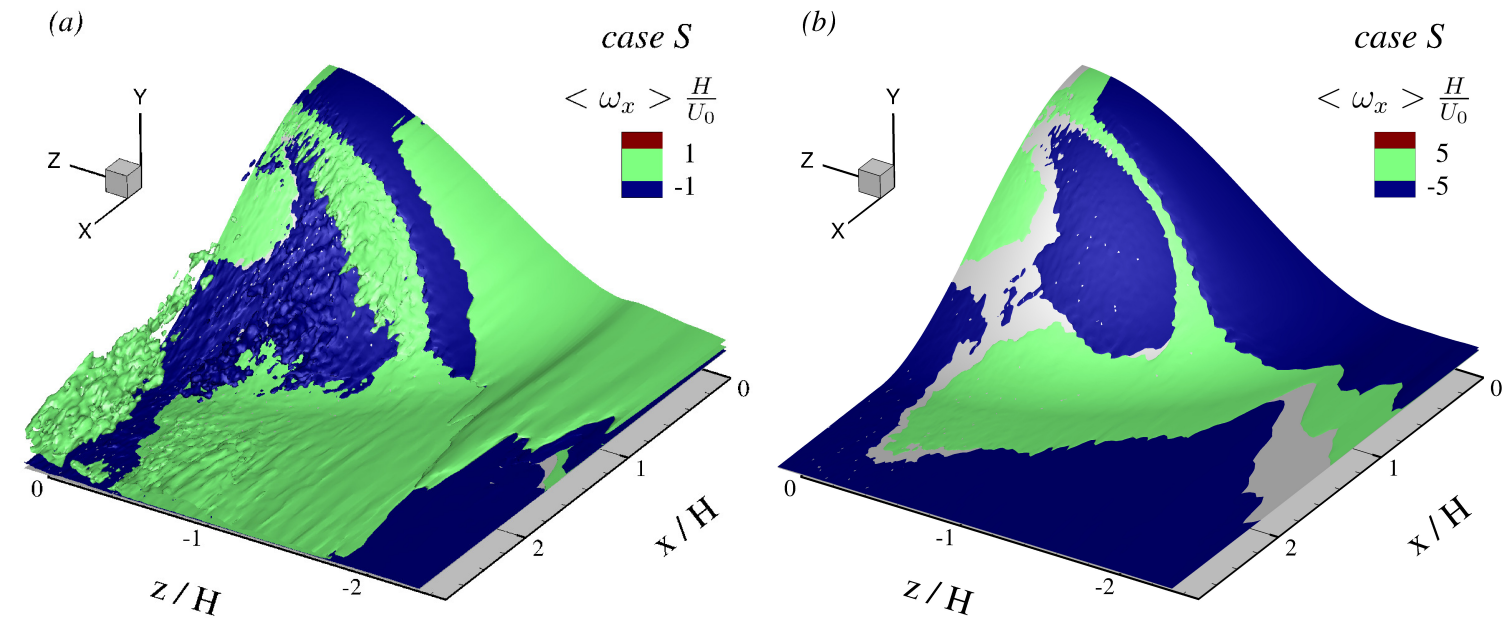

Fig. 17. Isosurfaces of the mean streamwise vorticity $\left(\left\langle\omega_{x}\right\rangle \frac{H}{U_{0}}\right)$ shown in the lee-side of the hill from the current LES case $S:(a)\left\langle\omega_{x}\right\rangle \frac{H}{U_{0}}= \pm 1$ and $(b)\left\langle\omega_{x}\right\rangle \frac{H}{U_{0}}= \pm 5$.

The flow topology at different wallnormal heights in Figs. 13 and 15, and the plot for $T K E$ depicting the separated shear layer above the recirculation region in Fig. 16, demonstrate the complexity of the three-dimensional pattern of this separational flow. The flow patterns vary significantly in all three directions. A complete three-dimensional view of the mean flow dynamics in the lee-side of the hill may be constructed that is also essen- 

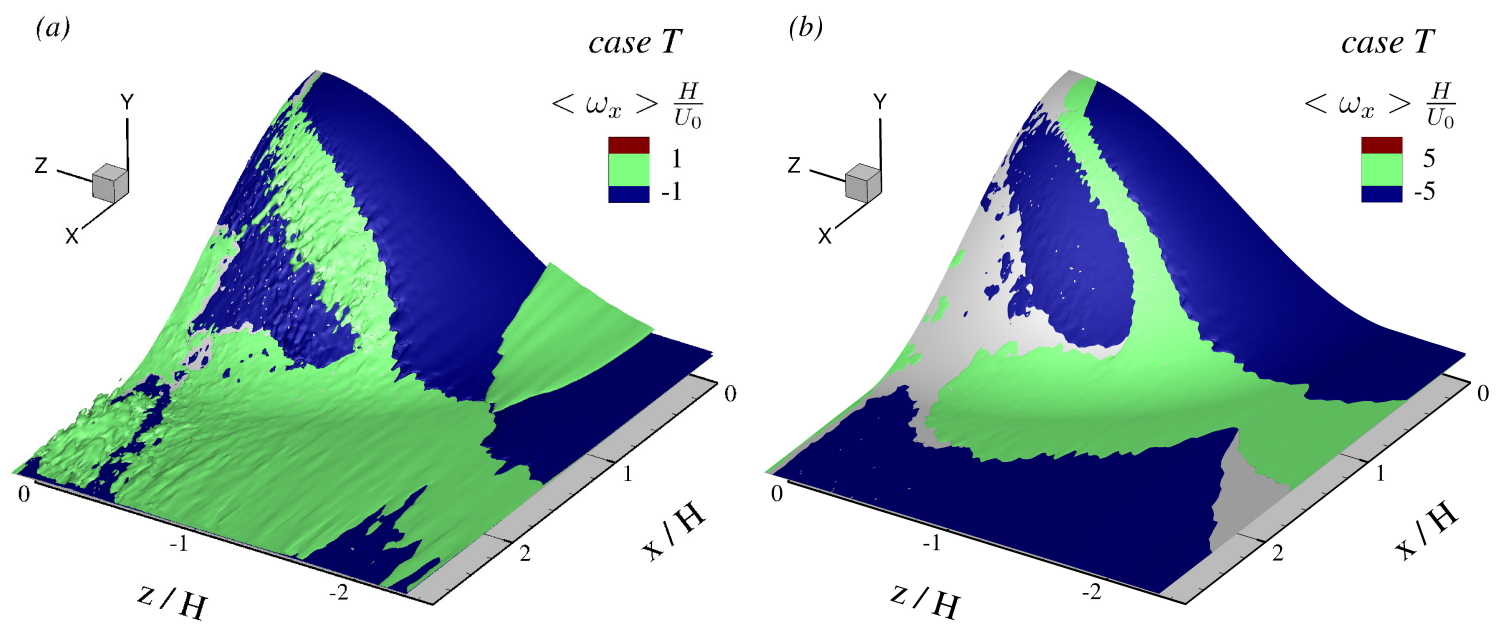

Fig. 18. Isosurfaces of the mean streamwise vorticity $\left(\left\langle\omega_{x}\right\rangle \frac{H}{U_{0}}\right)$ shown in the lee-side of the hill from the current LES case $T:(a)\left\langle\omega_{x}\right\rangle \frac{H}{U_{0}}= \pm 1$ and $(b)\left\langle\omega_{x}\right\rangle \frac{H}{U_{0}}= \pm 5$.

tial for the understanding of the flow recovery post reattachment in the wake of the hill. To help understand the dynamics of the vortical structures and the consequence of the upstream topological features on downstream flow evolution, isosurfaces of the scaled mean streamwise vorticity $\left\langle\omega_{x}\right\rangle=\frac{\partial\langle w\rangle}{\partial y}-\frac{\partial\langle v\rangle}{\partial z}$ were plotted for cases $S$ and $T$ in Figs. 17 and 18, respectively. The $x$ - component of the vorticity is representative of the secondary motions of the mean flow field. Because of the curvature of the surface underneath, $\omega_{x}$ continually changes sign in the wallnormal direction. Isosurfaces are plotted for a lower absolute value in frame $(a)$ and for a higher absolute value in frame $(b)$ of Figs. 17 and 18 to show the layers of $\omega_{x}$ changing sign of in that direction. Contours of scaled mean $\omega_{x}$ are shown at two cross-stream $(y, z)$-planes at chosen streamwise stations downstream of the crest of the hill for both cases in Fig. 19. Frames $(a)$ and $(c)$ show the contours for case $S$, and frames $(b)$ and $(d)$ for case $T$, at $x / H=1.5$ and 2.5 , which, respectively, belong in the flow separation region and the recovery region immediately downstream of the reattachment. The sign of $\left\langle\omega_{x}\right\rangle$ flips across the midspan $z=0$-plane in all these figures, and so, $\left\langle\omega_{x}\right\rangle$ is anti-symmetric w.r.t. this plane.

An intriguing perspective emerges from these figures. Because of the higher absolute values in Figs. 17(b) and 18(b), the isosurfaces depict high flow-gradient regions close to the wall. Dominant boundary-layer vorticity upstream of the hill is oriented in the spanwise direction. Following flow acceleration in the upstream side, and yawing of the streamlines, vorticity is reoriented in the streamwise direction. This is due to the lower streamwise flow acceleration experienced by fluid away from the midspan location; the spanwise pressure gradient establishes weaker spanwise flow along that direction close to the wall. Consequently at the crest of the hill, in Figs. 17(b) and 18(b), the dominant vorticity (the green patch underneath the blue patch) is positive closer to the wall in this half of the domain w.r.t. the midspan plane. For case $S$ in Fig. 17(a), an outer layer of positive vorticity (outer 
green patch) cowls the negative vorticity before separation in Fig. 17(b). For case $T$, this outer patch is only visible farther down the hill along the periphery of the hill geometry in Fig. 18(a). The vortices are seen to shed along the separation line for both cases (the green patch lifting off the surface of the hill). The shed vortices weaken downstream; this allows us to peep into the vortical structures underneath the shed vortices. In this half of the domain, the sense of the fluid curling into the vortices ejected from the focal node in the recirculation region in the topology maps shown in Figs. 13 and 15 has a negative sign. The vorticity of the converging flow into the focal node, which is ultimately ejected as vortices from close to the wall to the outer flow, is seen as the blue patch downstream of the shed vortices (green) for both cases. For case $T$, the recirculating flow between the focal node and the midspan plane has $\left\langle\omega_{x}\right\rangle>0$, which is seen in the isosurface plot as the green patch close to the midspan plane. This is not visible for case $S$, for which most of the recirculating flow converges on to the focal node (also see Fig. 13). Farther downstream, especially for case $S$, the negative vorticity in the inner region (frame $b$ in both figures) is impinged by the positive vorticity of the separated shear layer (in frame $a$ ), which is seen to reattach beyond $x / H \approx 2$.
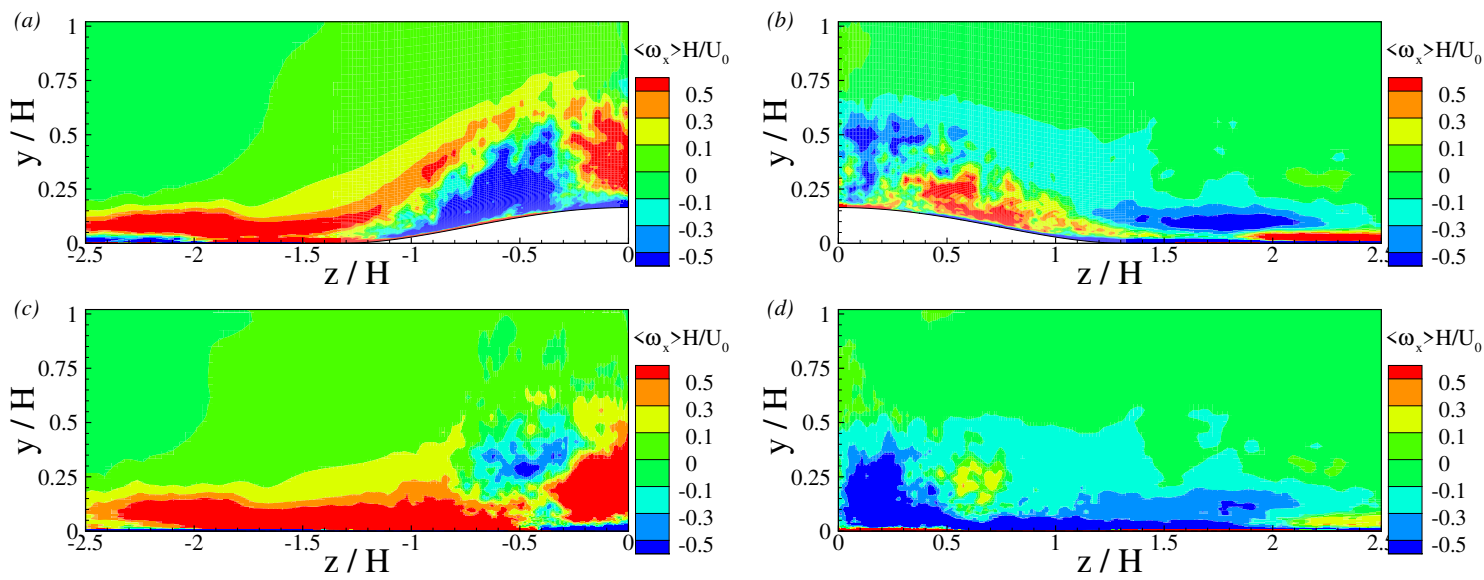

Fig. 19. Contours of mean streamwise vorticity $\left(\left\langle\omega_{x}\right\rangle \frac{H}{U_{0}}\right)$ plotted in wall-normal $(y, z)$-planes at chosen streamwise locations from the LES cases: $(a)$ and $(c) S$ and $(b)$ and $(d) T$. The streamwise locations are $x / H=1.5$ and 2.5 , respectively.

\subsubsection{Flow recovery}

A connection may be established between the mean separation dynamics and the flow recovery downstream of the reattachment point. The contours of $\left\langle\omega_{x}\right\rangle$ in Fig. 19 are helpful for this purpose. As $\left\langle\omega_{x}\right\rangle$ is antisymmetric w.r.t. the midspan plane, only half of this plane is shown for each case. Based on the flow structures observed in Figs. 17 and 18, three patches may be identified for both cases. In Fig. 19 for case $T$ in frame $(b)$ (case $S$ in frame a) at $x / H=1.5$, the blue (red) patch close to the midspan plane represents the vortices 

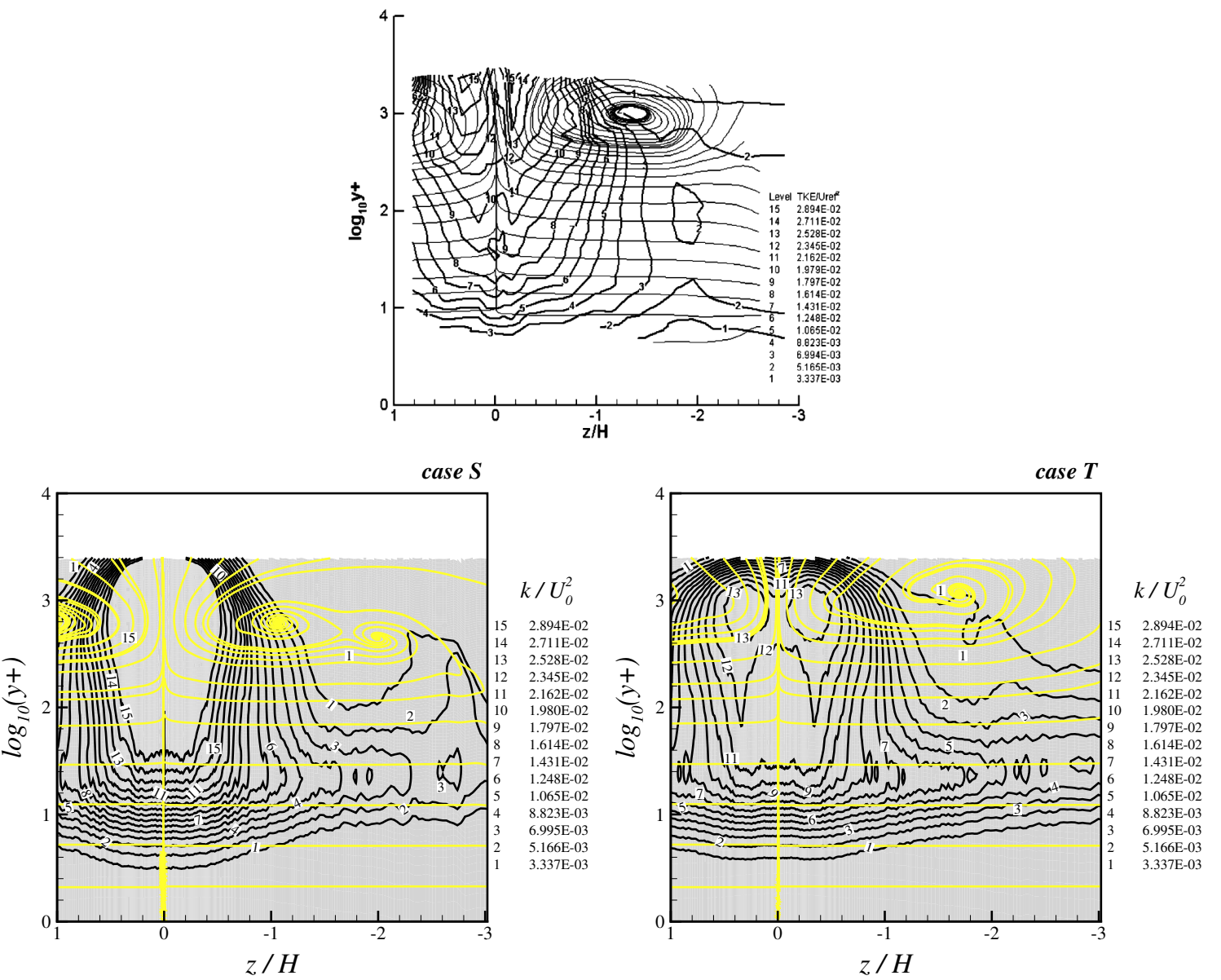

Fig. 20. Contours of $T K E$ in the cross-stream $(y, z)$-plane at $x / H \approx 3.63$ from the experiment [38] and from current LES cases $S$ and $T$. Secondary flow is also depicted by showing the streamlines from the in-plane mean velocity vectors.

shed from the separation node, an extended red (blue) patch depicting the recirculating flow close to the wall as well as vortices ejected from the focal node farther away from the wall as in Fig. 18 (17), followed by an outer blue (extended red) patch showing the upstream realigned boundary-layer vorticity. The vorticity shown as the red (blue) patch for case $T(S)$ between the two blue (red) patches is especially interesting. Underneath the strong vortical structures depicted by the blue (red) contours for the case $T(S)$ close to the midspan plane, a very thin layer of vorticity with the opposite sign is visible very close to the wall, and gets thicker down hill in the spanwise direction. The thin layer is representative of the recirculating flow with streamlines that do not converge on to the focal node in Fig. 13. The recirculation region is thicker for case $S$, and the vortices ejected from the focal node are not differentiable. On the other hand, for case $T$, the recirculation layer is thinner and the ejected vortices from the focal node are weaker than in case 
$S$. The outer red layer of realigned upstream boundary-layer vorticity is much stronger for the case $S$ at $x / H=1.5$. The prolonged separation for case $S$ is due to the upstream vorticity being retained up to the point of reattachment. In the absence of any turbulence at the inlet of the domian, the separation for case $S$ is akin to a laminar separation compared to case $T$, for which, the inflow turbulence increases the flow gradients close to the wall, thereby, suppressing the separated shear layer. The flow gradients are lower for case $S$ after the initiation of separation, resulting in a thicker separated shear layer (also seen in Fig. 16), and a recirculation layer below it (Fig. 12). This is investigated later in more detail. The flow farther downstream at $x / H=2.5$ for both cases in Figs. $19(c)$ and 19(d), i.e., in the near wake flow recovery region, is dominated by the vorticity from the separation line (the patch close to the midspan plane) and the upstream reoriented boundary-layer-vorticity (outer patch), discussed earlier and also visible in Figs. 17 and 18. It should be noted that, for case $S$, this location is just downstream of the reattachment node at $x / H \approx 2.42$.

Beyond the reattachment point, Fig. 20 shows for both LES cases the secondary flow in the recovery region, at $x / H \approx 3.63$ in the cross-stream $(y, z)$-plane. Also shown is the secondary flow in the experiment. Inplane $T K E$ contours with the same values are also shown. A logarithmic scale is used in the wall-normal direction. The plot in local viscous wall units clearly identify the location of the flow features in the turbulent wake of the hill. In Fig. 19, for both LES cases the upstream boundary-layer-vorticity is seen to merge with the vorticity from the separation node for both cases. Also for both LES cases, the mean streamwise vorticity from the separation line and the corresponding secondary flow dissipate with downstream distance. For the experiment and for case $T$ the upstream boundary-layer-vorticity dominates. The center of the secondary motion is located farther away from the midspan plane for case $T$ at $z / H \approx-1.8$ instead of $z / H \approx-1.5$ for the experiment. For case $T$, good agreement with the experiment is evident for the $T K E$ contours also, although the values of $T K E$ are slightly lower (the highest contour value of 0.02894 is not obtained). The $T K E$ is lower in the plane of symmetry possibly due to the convection of fluid from near the symmetry plane to the outer regions as may be seen from the sense of the secondary flow in the wake of the hill [16]. The two lobes in the TKE contours on either side of the midspan plane are obtained at slightly lower wall-normal locations for case $T$; however, as is discussed later, this could be due to the under-predicted friction velocity at this location. Interestingly, for case $S$, in the absence of ambient turbulence activity, in the wake of the hill, a vortex pair appears beyond the reattachment point in the inner parts of the boundary layer, which strengthens and then dissipates. The inner pair of vortices have the same sign as the outer vortex which can be clearly identified in the plot for case $S$. These are likely due to the lack of transport of momentum by turbulence from outer regions towards to the symmetry plane in the wake of the hill. For case $S, T K E$ values are higher at same wall-normal heights close to the midspan plane despite the contour characteristics being largely different.

In most of earlier computational studies, the flow field at location $x / H \approx 3.63$ is shown to be in good agreement with the experimental results. Author in [15] speculated that the good agreements 'could be a matter of chance'. The agreement with the experiment is rea- 


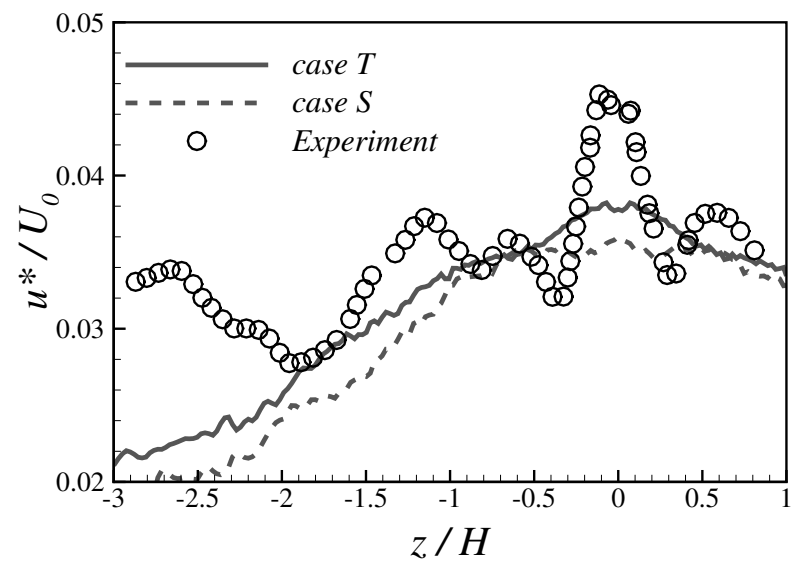

Fig. 21. Friction velocity in the spanwise direction in the flow recovery region at $x / H \approx 3.63$ from current LESs compared with the experiment [38].

sonably good. Further comparisons are performed, first by plotting the scaled skin-friction velocity $\left(u * / U_{0}\right)$ in the spanwise direction in Fig. 21. This quantity is sensitive to adequate resolution in all three directions. With respect to the inlet wall unit, $\Delta x+\approx 135$ at this streamwise station, i.e., nominal for a resolved LES. Taking this into consideration, $u *$ is in reasonably good agreement with the experiment. The match for the case $T$ is better due to the higher turbulence fluctuations associated with the inlet condition for this case. Evidently, for both cases $S$ and $T$ the agreement is best in the vicinity of the symmetry plane where the spanwise resolution is maximum. The agreement is reasonable within the spanwise extent of the hill, beyond which the computed $u *$ is underpredicted; the discrepancy being significant due to the lower grid resolution and stretching in that direction.

The effect of the inflow turbulence on the flow recovery downstream of the hill is studied by plotting the wall-normal profiles of mean streamwise $(\langle u\rangle)$ and spanwise $(\langle w\rangle)$ velocities at several spanwise locations at $x / H=3.63$ in Fig. 22 and of $T K E$ and cross resolved turbulent stress term $\left\langle u^{\prime} v^{\prime}\right\rangle$ in Fig. 23. Figures 22 and 23 include profiles from the LES case $T$ (solid lines), case $S$ (dashed lines) and experiment (symbols). The profiles at increasing distance from the midspan plane are coordinate-shifted for clarity. Overall, good quantitative agreement with the experiment is obtained for case $T$, especially at stations closer to the plane of symmetry at $z / H=0$; the discrepancies increase farther away from this plane, possibly due to coarser grid resolution in both streamwise and spanwise directions. At the last two spanwise stations $(x / H \geq 1.1)$ farthest from the midspan plane, the $\langle u\rangle$-profiles for the case $T$ are fuller than the experimental profiles. Due to the presence of the hill, the streamwise momentum is reduced in the lee-side of the wake which is reflected in the inflectional profiles of $\langle u\rangle$ at $z / H=0.32$ and 0.65 . The deficit is clearly more pronounced for case $S$, for which strengthening of the inner vortex pair in Fig. 20 increases momentum deficit closer to the midspan plane at this streamwise station. This also results in the poorer 
(a)

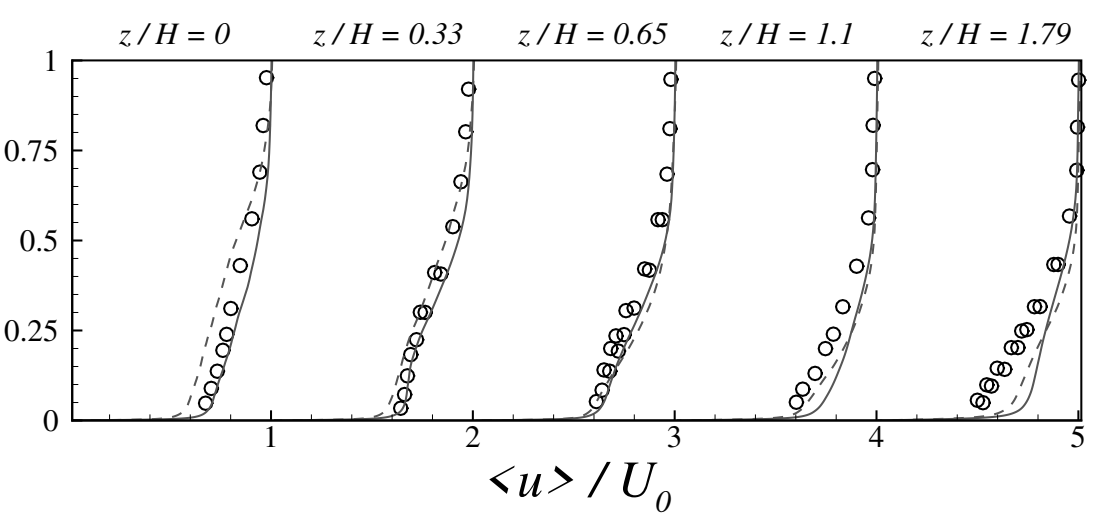

(b)

b) $\quad z / H=0 \quad z / H=0.33 \quad z / H=0.65 \quad z / H=1.1 \quad z / H=1.79$

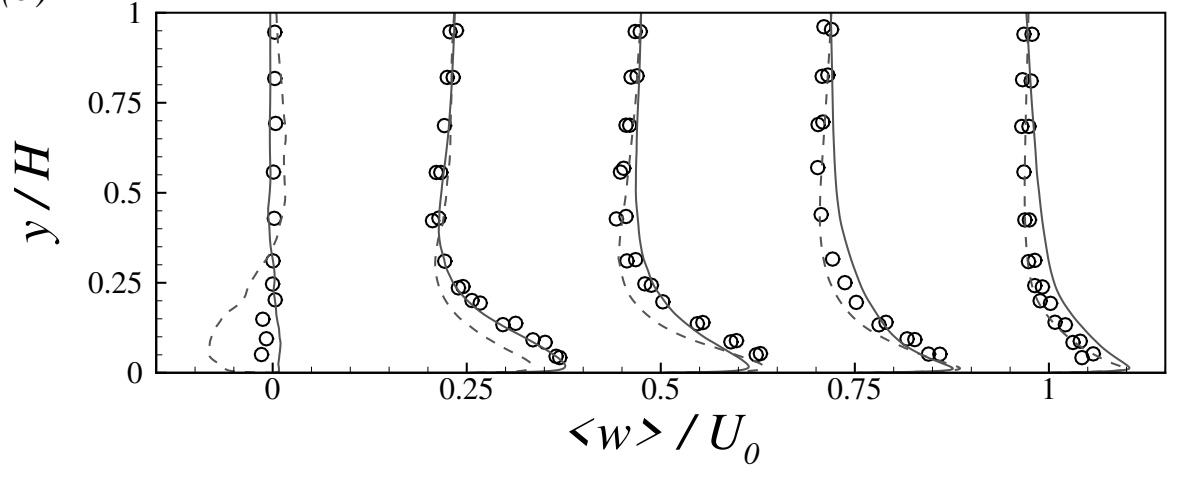

Fig. 22. Wallnormal profiles of the mean $(a)$ streamwise $\left(\langle u\rangle / U_{0}\right)$ and $(b)$ spanwise $\left(\langle w\rangle / U_{0}\right)$ velocities at $x / H=3.63$ from current LES case $S$ (dashed lines) and case $T$ (solid lines) compared with experiment (symbols) [4]. For clarity, each profile of $\langle u\rangle / U_{0}$ and $\langle w\rangle / U_{0}$ has been shifted by the nondimensional units 1 and 0.25 , respectively.

comparisons with experiment for case $S$. Profiles of $\langle w\rangle$ are also inflectional at spanwise stations away from the midspan location. Close to the wall, mean fluid motion is away from the symmetry plane (also see Fig. 13, and $\langle w\rangle>0$ close to the wall for $z / H>0$ ); and farther away from the wall, fluid moves away from the symmetry plane as the profiles of $\langle w\rangle$ change sign in the wall-normal direction. This shift in momentum transfer results in the inflectional profiles of $\langle u\rangle$.

As may be expected for case $S$, the second order statistics presented in Fig. 23 are in lesser agreement with the experimental profiles, especially at spanwise stations close to the symmetry plane; both $k$ and $\left|\left\langle u^{\prime} v^{\prime}\right\rangle\right|$ are significantly overpredicted for this case at $z / H=0$ and 0.33 . Also, higher values of $T K E$ are obtained at $z / H=0.32$ instead of at $z / H=0$ for both cases. Authors in [16] attributed this to the transport of turbulent fluid from the separation region to the wake by the $\langle w\rangle$-component of the mean momentum. This phenomenon is further enhanced for case $S$ due to the previously mentioned inner vortex pair, and therefore, both $k$ and $\left|\left\langle u^{\prime} v^{\prime}\right\rangle\right|$ are higher. However, at outer spanwise locations, i.e., at $x / H=0.65,1.1$ and 1.79, the profiles from both LESs are in good agreement with the 

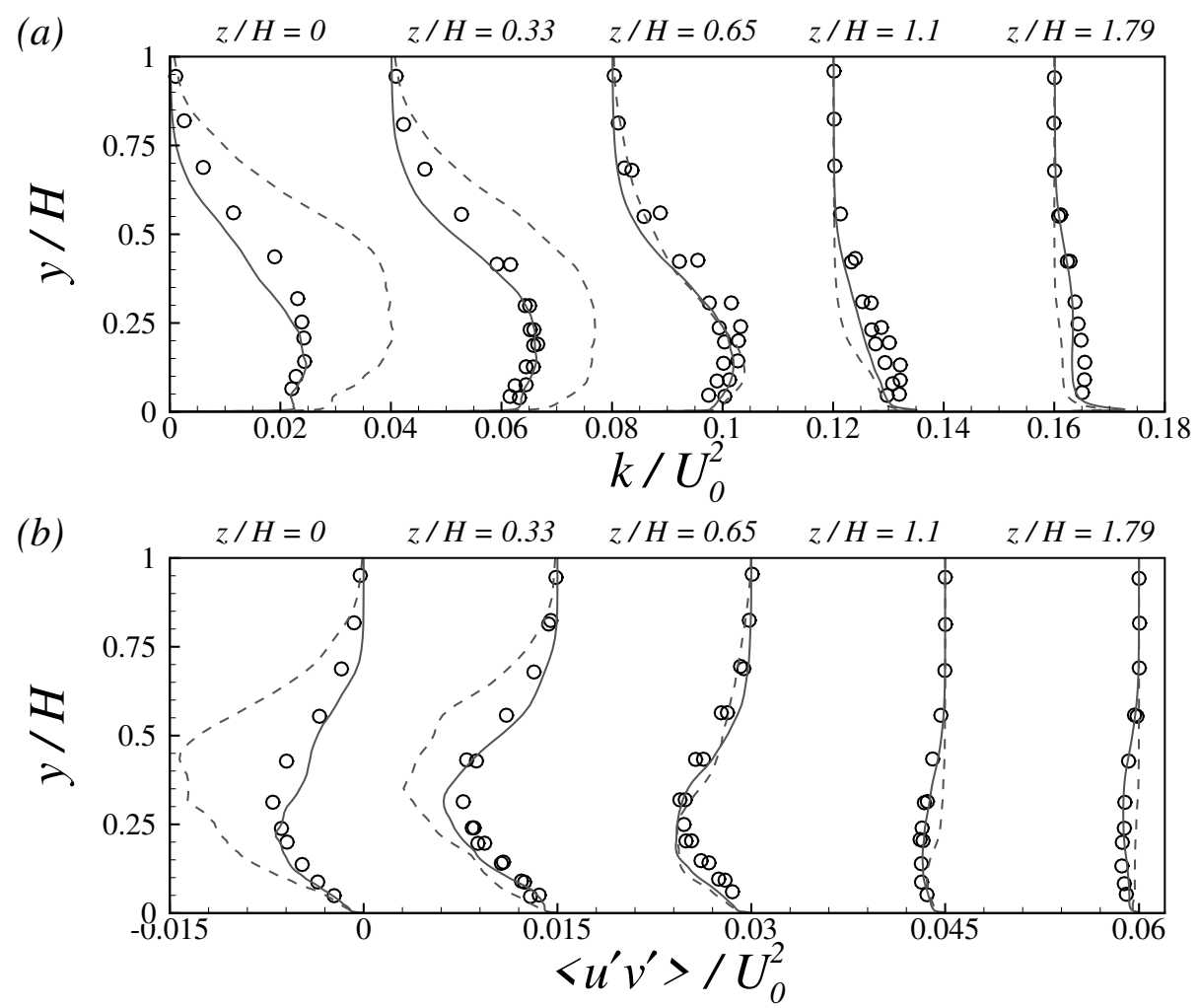

Fig. 23. Wallnormal profiles of the scaled (a) TKE $\left(k / U_{0}^{2}\right)$ and $(b)$ resolved turbulent stress component $\left(\left\langle u^{\prime} v^{\prime}\right\rangle / U_{0}^{2}\right)$ at $x / H=3.63$ from current LES case $S$ (dashed lines) and case $T$ (solid lines) compared with experiment (symbols) [4]. For clarity, each profile of $k / U_{0}^{2}$ and $\left\langle u^{\prime} v^{\prime}\right\rangle / U_{0}^{2}$ has been shifted by nondimensional units 0.04 and 0.015 , respectively.

experiment. Predictions for case $T$ are much more reliable for this flow in the separational, post-reattachment recovery regions. It was noted by the $L L$ group in [16], that the second order statistics are more difficult to predict for this flow even for resolved simulations. Excellent agreement of case $T k$ and $\left\langle u^{\prime} v^{\prime}\right\rangle$ profiles with the experimental profiles at all spanwise stations demonstrates the efficacy of the current simulations. Because case $T$ results are noticeably better than for case $S$, instantaneous flow fields in the next section are presented for case $T$ only.

\subsection{Results: Instantaneous flow \& Spectra}

Figure 24 shows the steamwise fluctuation $\left(u^{\prime}\right)$ contours at two extracted planes at chosen wall-normal distances from the bottom wall from the case $T$. For frames $(a)-(d)$ and frames $(e)-(h)$, the chosen wall-normal distances from the bottom wall correspond to $\Delta y+\approx 150$ and 1500, respectively, w.r.t. the viscous wall unit at the inlet. Frames $(a)$ and $(e)$ are extracted at the same time instant (say, $t_{0}$ ), while the next three frames are at irregular 

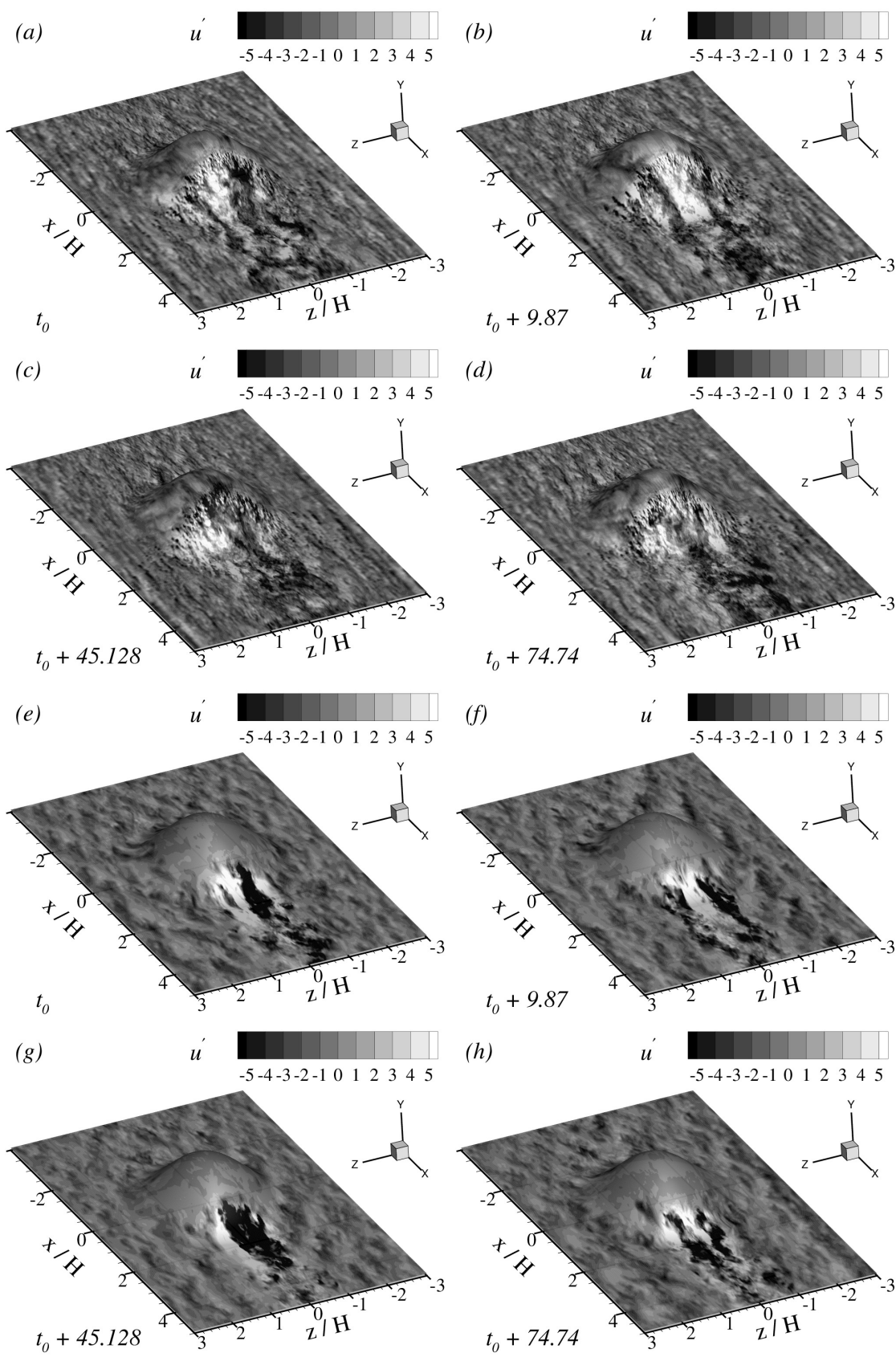

Fig. 24. Contours of instantaneous streamwise velocity fluctuation $\left(u^{\prime}\right)$ in extracted wall-parallel planes at chosen heights above the lower wall for the LES case $T$. The extracted planes are located at heights: $(a)-(d) y+\approx 150$, and $(e)-(h) y+\approx 1500$ w.r.t. the viscous wall unit at inlet.

intervals (shown in the corresponding frames in nondimensional time unit $H / U_{0}$ ) w.r.t. $t_{0}$. Only the $u^{\prime}$ component is shown; this helps to identify the long streaky vortical structures in 
the wake of the hill. The dark patches indicate negative fluctuations, that is, slower moving fluid compared to the mean flow. Close to the wall, in frames $(a)-(d)$ the flow field contains significantly smaller scale high intensity fluctuations compared to farther away from the wall in frames $(e)-(h)$. For example, at the onset of separation, very fine scale fluctuations are visible closer to the wall that are absent farther away from the hill. In the upstream-side FPG region, amplitude of these high-frequency fluctuations are somewhat attenuated (flow does not relaminarize) which reappear upon pressure gradient turning adverse, and further downstream at the onset of separation. The flow does not separate even instantaneously in the plane corresponding to frames $(e)-(h)$ (see Fig. 15). Intriguingly in this plane though, streamwise long streaky structures can be clearly identified that transport momentum to and from the outer separated shear layer.
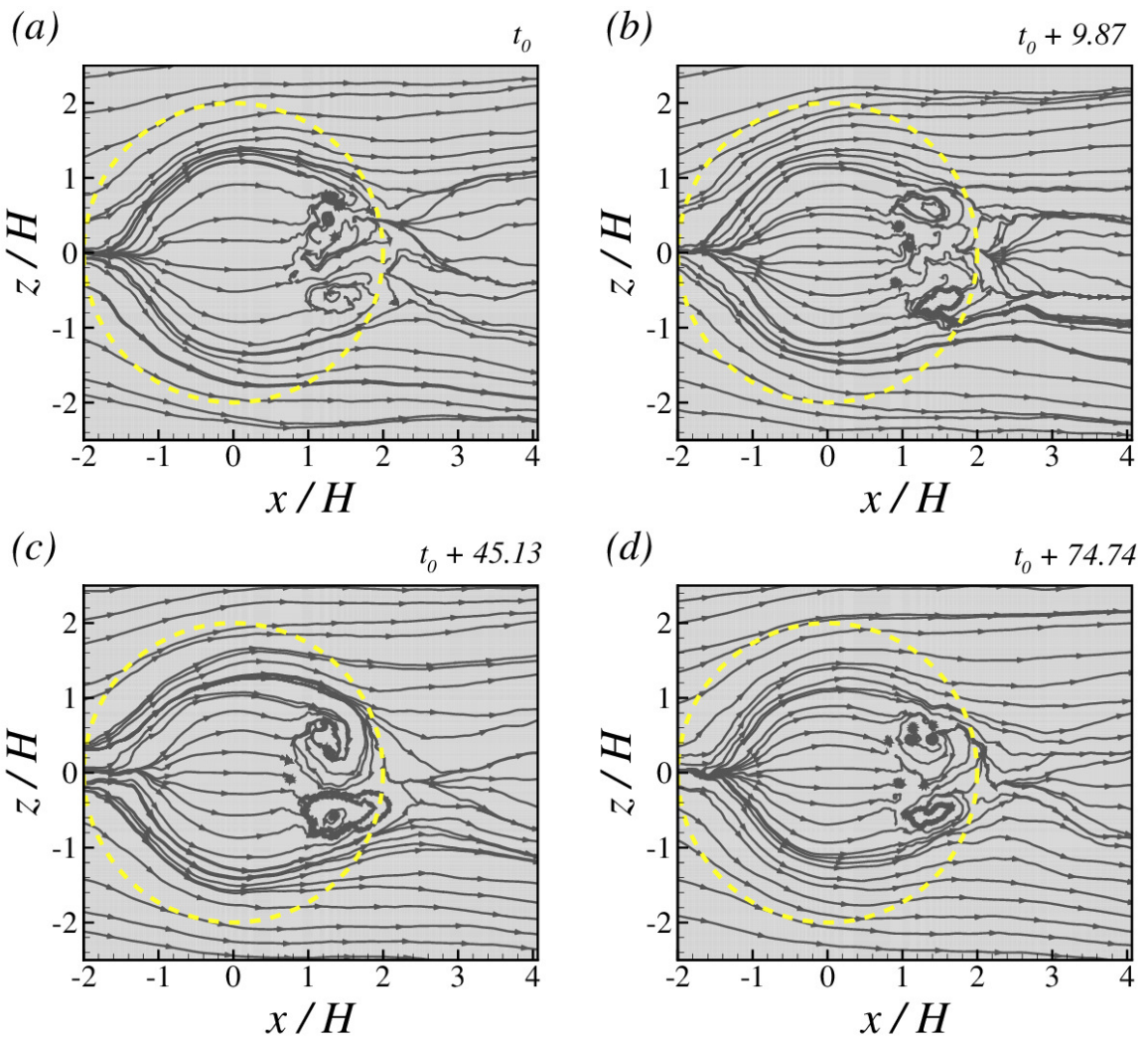

Fig. 25. Instantaneous streamlines from inplane velocity fields in an extracted wall-parallel plane projected onto the $(x, z)$-plane as in the experimental results of Fig. 14, for current LES case $T$. The time instants are same as in Fig. 24. The circle shows the outer circumference of the hill. The plane is at a height $y+\approx 150$ (w.r.t. the viscous wall unit at inlet) above the bottom wall.

The streaky structures intermittently arise from one side of the hill to the other at irregular intervals, as no clear time-periodicity could be identified from the animations of the fluctuation fields, or the spectra (discussion to follow) at different wall-normal heights. 
Occasionally, a high-speed streak in one side of the hill is accompanied by a low-speed streak on the other, which alter their positions at later time instants. At some instants, a high-speed (low-speed) streaky structure may arise in between two low-speed (high-speed) streaks, as in frame $(f)$. Also, as in frame $(h)$, multiple pairs of streaky vortical structures of alternating sign may appear. These events are intermittent, as these arise, are sustained for some time, and then either wash away downstream, or dissipate. That these structures are irregular and can not be associated with periodic vortex shedding is reflected by the irregular time intervals of their appearance in these frames. By plotting isosurfaces of the pressure fluctuations, researchers in [16] found that hair-pin shaped vortical structures often ride upon these streaky structures, particularly the low-speed streaks in the wake of the hill. The aforementioned patterns of low and high-speed streaky structures in frames $(e)-(h)$ are not apparent close to the wall, although imprints of these vortical structures in the outer flow may be identified close to the wall in frames $(a)-(d)$; however, these are only identifiable at closer inspection. These images show that the instantaneous flow fields, despite being largely different, provide a very complex, yet clear time-mean flow pattern discussed earlier. Further analysis of the instantaneous vortex fields, e.g., through different decomposition methods is out of scope of our computational capacity.

Streamlines drawn from the instantaneous velocity fields in the same wall-parallel plane and at the same instants as in Figs. 24(a)-24(d), projected onto the horizontal $(x, z)$-plane, are shown in Fig. 25. This figure is similar to Fig. 15, the only exception being the use of instantaneous velocity fields instead of the mean at only one wall-parallel plane. Noticeable differences between the characteristics of Fig. 25 and Fig. 15 bring out the complexity of the instantaneous flow field compared to the mean flow field. Unlike for mean flow and the critical features of the topology maps in Figs. 13 and 15, such features are intermittent in the instantaneous fields. The focal nodes in the separation region, for example, are sometimes clearly present in both halves (frame $b$ ), and sometimes clearly identifiable in only one half of the hill (frame $c$ ), while at other instants they can not be identified clearly; other complex features are absent from the mean flow topology maps. The intensity, shape and position of the flow features also change significantly between time instants. The flow recovery region downstream of reattachment is also affected by the occurrence of these upstream flow features. Another interesting feature may be identified by comparing the $u^{\prime}$ contours in Figs. 24(e)-24(h) with the instantaneous streamlines at corresponding time instants in Figs. 25(a)-25(d). The low-speed streaky structures farther away from the wall are associated with the instantaneous focal nodes whose position, width and strength vary with those of the low-speed streaky structures. Fluid curling up into the focal nodes results in the momentum deficit that shows up as the low-speed streamwise streaky structure in $u^{\prime}$-fluctuation fields.

Frequency spectra are plotted against the nondimensional frequency $f H / U_{0}$ in the left and right columns of Fig. 26 for the $u$ - and $w$ - fluctuations, respectively. The streamwise location is the same as in Figs. 20-23, in the flow recovery region in the midspan plane $(z / H=0)$ in the wake of the hill. The spectra from both the current LESs and experiment (symbols) are shown in frames $(a)$ and $(b)$ at $y / H \approx 0.36$. Frames $(c),(d)$, and $(e),(f)$ in 

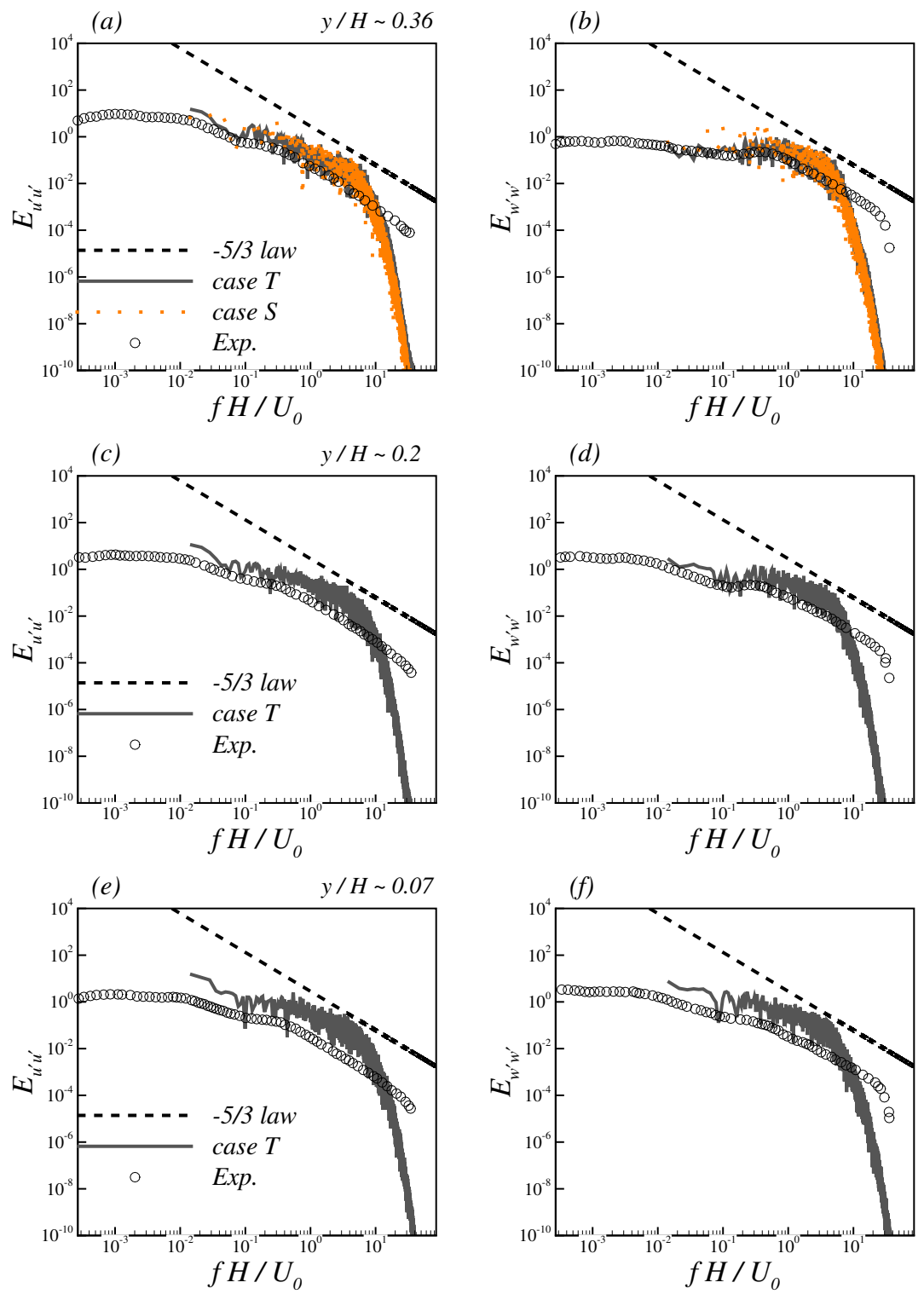

Fig. 26. Frequency spectra of the streamwise (left) and spanwise (right) velocity fluctuations in the midspan $(z=0)$ plane at $x / H=3.63$ compared with experiment (symbols): (a) $-(b) y / H=0.36$; (c) $-(d) y / H=0.2 ;(e)-(f) y / H=0.07$. The dashed lines correspond to $-5 / 3-$ law.

Fig. 26, show the corresponding spectra at locations closer to the wall, at $y / H \approx 0.2$ and 0.07 , respectively, in which only the results from case $T$ (results for case $S$ at all locations shown in the figure) are compared with the experiment. All these wall-normal locations belong to the region where high $T K E$ values are obtained in Fig. 23. Data were recorded for a time span of $\approx 282.05 \frac{H}{U_{0}}$. To obtain the spectra over a broad range of frequencies and at the same time to acquire some smoothness, the whole time span of the recorded data 
was split into three equally overlapping chunks of equal time spans. A Hann window was applied to each chunk of the data and then a Fast Fourier Transform (FFT) was applied to each chunk. The spectra shown are the mean of the three separately calculated FFTs. The spectra from the experiment extend over a much larger range of frequency compared to the spectra obtained from the current LESs. The agreement with the experiment is generally good at all wall-normal locations. The spectra from the current LESs drop off faster beyond $f H / U_{0} \approx 8$ because of the relatively large grid spacing. Reasonable quantitative agreement with the experiment is obtained also at lower frequencies $f H / U_{0}<0.1$. The slopes of the spectra from case $T$ (and also case $S$ in frames $a$ and $b$ ) are consistent with the isotropic range satisfying the $-5 / 3$ law over at least a decade and a half of the logarithmic frequency scale for the $u$-component, and for at least a decade of the frequency range for the $w$ component. Amplitudes of the spectra from the current LESs slightly overshoot the spectra from the experiment at all wall-normal locations; the difference in amplitude increases close to the wall. This indicates that the over-prediction in the LESs is likely due to the numerical noise associated with the discretization error and the resolved turbulence at the smallest grid unit; despite increasing wall-normal grid resolution close to the wall owing to the grid stretching in that direction, the streamwise and spanwise resolutions remain the same. It is noted that no distinct peak is obtained at $f H / U_{0} \approx 0.41$, especially in the spectrum for the $w$-fluctuations at any of the wallnormal locations, as was the case for the precursor simulation in Fig. 8 at $y+\approx 1500$; the latter was attributed to the imposed streamwise periodicity in a short streamwise domain used for the precursor simulation. Therefore, no discernable effect of the time-periodicity of inlet turbulence is retained this far downstream of the inflow of the computational domain. 


\section{Effect of inlet turbulence}
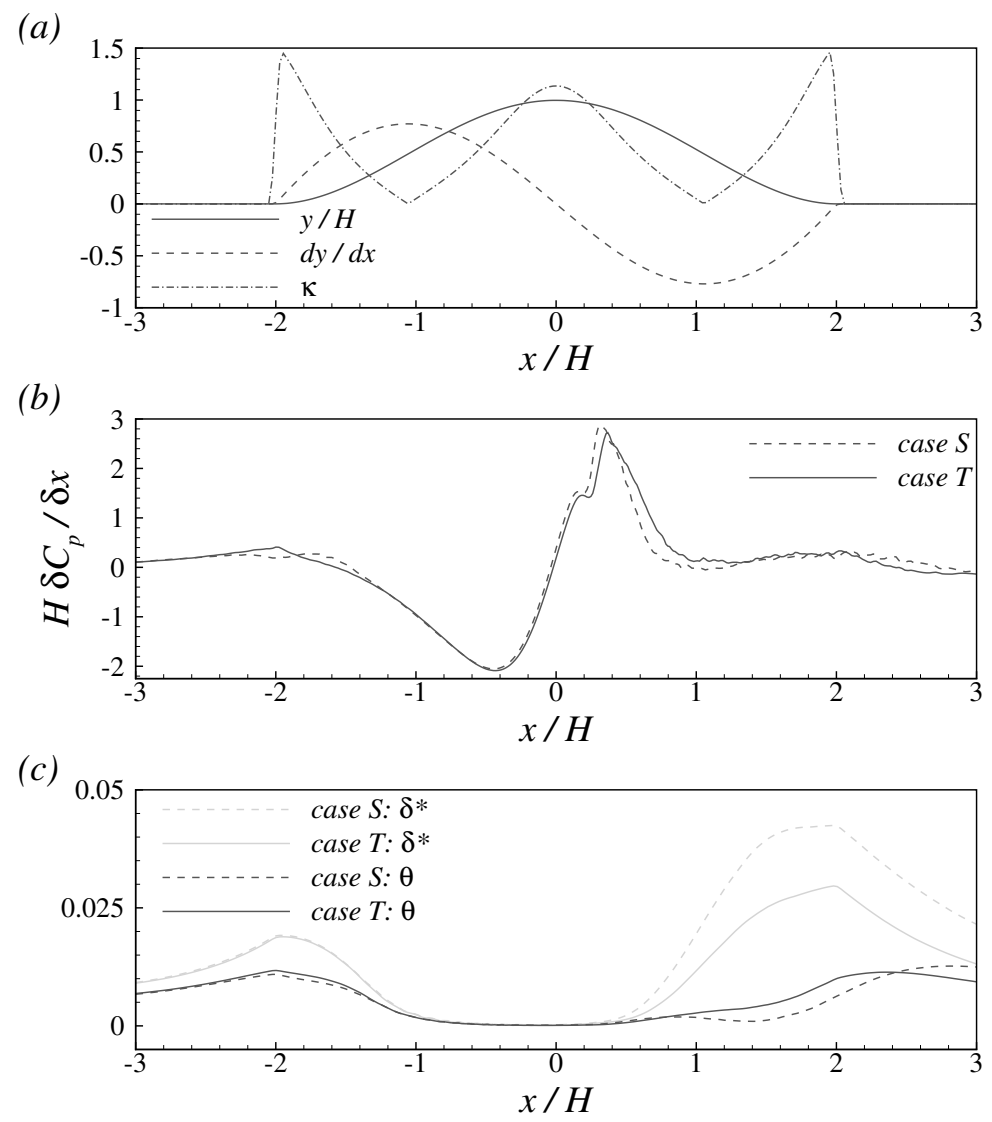

Fig. 27. Streamwise profiles of $(a)$ the hill surface, its slope $\left(\frac{\partial y}{\partial x}\right)$, and curvature $\kappa=\frac{\left|\frac{\partial^{2} y}{\partial x^{2}}\right|}{\left[1+\left(\frac{\partial y}{\partial x}\right)^{2}\right]^{1.5}}$, (b) the streamwise pressure gradient $\left(H \frac{\partial C_{p}}{\partial x}\right)$ on the bottom wall $(c)$ displacement thickness $(\delta *)$ and momentum thickness $(\theta)$ at the bottom wall in midspan plane $(z / H=0)$.

Several interesting observations were made in Sec. 3 on the comparison of the mean flow in cases $T$ and $S$ with their counterpart in the experiments. The difference between the two LESs is due to the turbulence injected at the inlet in case $T$. In spite of the very different incoming flows, the $C_{p}$ distribution in Figs. 10 and 11 as well as the flow topology maps in Figs. 13 and 15 showed similarities in the flows upstream of separation. The onset of separation was also found to be relatively similar in the two cases. On the other hand, the flow downstream of separation up to the near wake and in the recovery region was found to be significantly different for the two LESs. The incoming turbulence in case $T$ affects the flow only downstream of the flow separation. Owing to the development of an internal layer in the upstream-side slope of the hill the effect of the incoming turbulence on the flow upstream of the separation zone is not significant. The effect of the viscous turbulent 
stresses upstream of separation is to marginally delay its onset. These intriguing aspects are investigated here in more detail.

In Fig. 27(a), the streamwise profile, slope $\frac{\partial y}{\partial x}$, and curvature $\kappa=\frac{\left|\frac{\partial^{2} y}{\partial x^{2}}\right|}{\left[1+\left(\frac{\partial y}{\partial x}\right)^{2}\right]^{1.5}}$ of the hill geometry, are plotted in the midspan plane. The along-flow hill profile in the upstream side is initially concave as the slope of the profile first increases, reaches a maximum at $x / H \approx-1.06$ (at this point the curvature $\kappa$ is minimum) and then becomes negative (i.e., the curvature turns convex). Due to symmetry, the profile curvature switches back from convex to concave again at $x / H \approx 1.06$. Figure $27(b)$ shows the non-dimensional streamwise pressure gradient $\left(H \frac{\partial C_{p}}{\partial x}\right)$ plotted in the bottom wall along the midspan plane for both LES cases. The pressure gradient profiles for both cases are very similar throughout the extent of the domain in this plane, except for a slight difference after the initiation of separation up to the reattachment point. The turbulent inflow condition has minimal influence on the wall pressure gradient. Downstream of the inflow plane, the flow experiences an APG, which increases up to $x / H \approx-2$. Up on the hill profile, the pressure gradient reduces with downstream distance; although the switch in curvature takes place at $x / H \approx-1.06$, the near-wall pressure gradient switches from APG to FPG at $x / H \approx-1.5$ (there is a slight difference between the two cases). Change in the pressure gradient precedes a switch in the surface curvature, which induces the pressure gradient in the flow. Beyond the maximum FPG obtained at $x / H \approx-0.43$, the pressure gradient increases again to turn adverse at $x / H \approx-0.02$. The APG that eventually leads the flow to separate is underway upstream of the crest of the hill. The effect of the pressure gradient remains consistent throughout the boundary-layer thickness and is shown by plotting the integral parameters, displacement thickness, $\delta *(x)=\int_{0}^{\eta_{j}}\left(1-\frac{\left\langle u_{t}(x, \eta)\right\rangle}{\max \left\langle u_{t}(x)\right\rangle}\right) d \eta$, and momentum thickness, $\theta(x)=\int_{0}^{\eta_{j}} \frac{\left\langle u_{t}(x, \eta)\right\rangle}{\max \left\langle u_{t}(x)\right\rangle}\left(1-\frac{\left\langle u_{t}(x, \eta)\right\rangle}{\max \left\langle u_{t}(x)\right\rangle}\right) d \eta$. Here, $\eta$ is the wall-normal coordinate, and $\eta_{j}$ denotes the wall-normal location where $\left\langle u_{t}(x, \eta)\right\rangle=\max \left\langle u_{t}(x)\right\rangle$. Differences between the two LES cases are only noticeable after the separation is initiated. Up until that point, the mass and momentum deficits are same for both LESs; the effect of streamwise pressure gradient is experienced proportionately throughout the whole boundary layer in both LESs. The deficits reduce in the upstream side of the hill and only start increasing beyond separation onset.

Local profiles of the inplane components of the mean velocity $(\langle u\rangle$ and $\langle v\rangle)$ are plotted at various streamwise stations in the midspan plane in Fig. 28. Profiles of the three nonzero components of the resolved turbulent stresses, $\left\langle u^{\prime} u^{\prime}\right\rangle,\left\langle v^{\prime} v^{\prime}\right\rangle$, and $\left\langle u^{\prime} v^{\prime}\right\rangle$ are plotted in Fig. 29 at the same locations as in Fig. 28. The chosen streamwise locations range from upstream of the hill at $x / H=-3$ to a location downstream of the separation onset, at $x / H=0.5$. The locations are chosen to show the profiles at streamwise stations of interest in Fig. 27; locations in the first row of frames in the Figs. 28 and 29 correspond to the APG region in the upstream side of the hill up to the point where pressure gradient turns favorable at $x / H \approx-1.5 ; x / H=-1.11$ and -1 in the second row of frames are in either side of $x / H \approx-1.06$, where the hill curvature turns convex; $x / H=-0.48$ and -0.26 in 

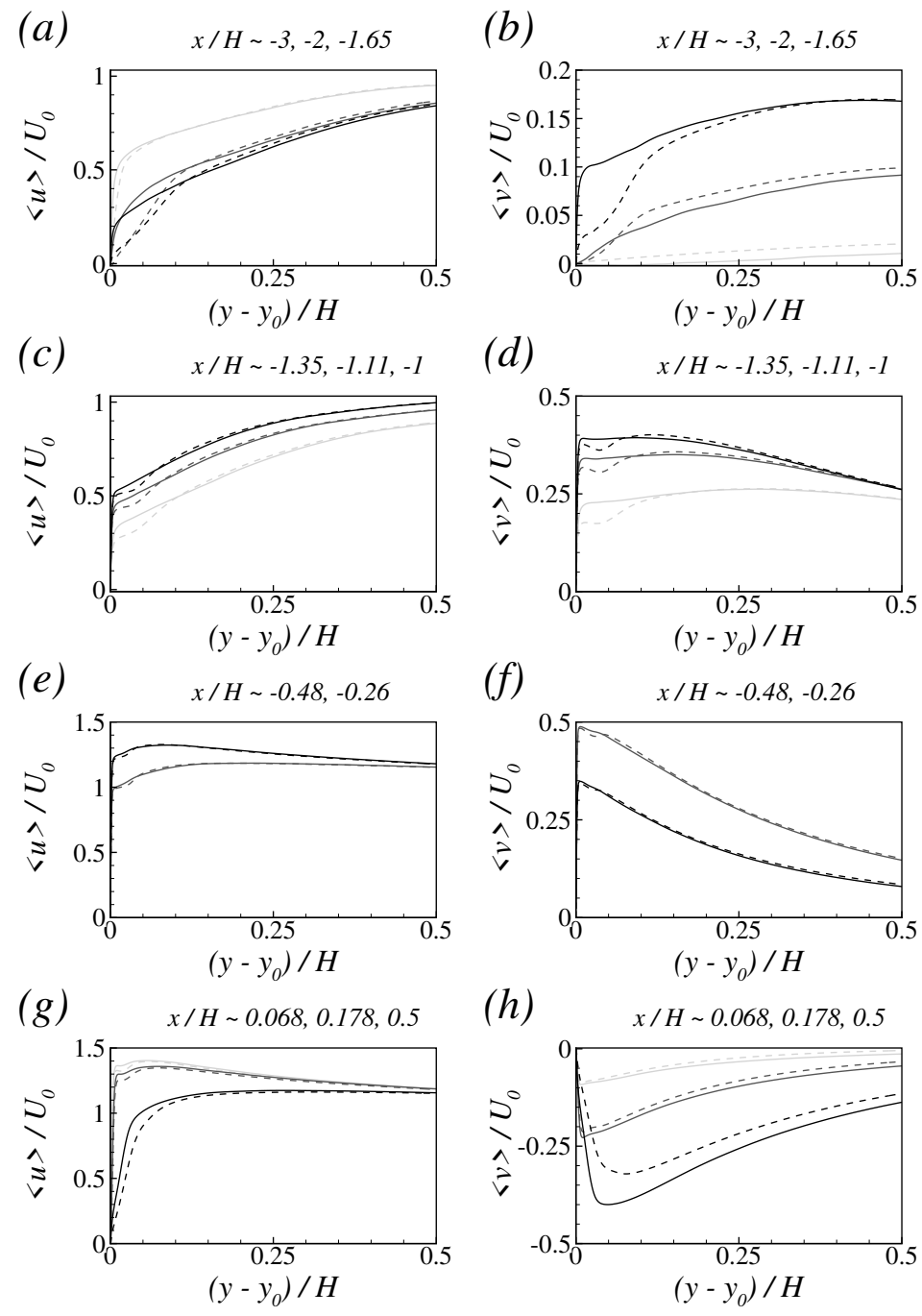

Fig. 28. Local profiles of $\langle u\rangle / U_{0}$ (frames $a, c, e, g$ ) and $\langle v\rangle / U_{0}$ (frames $b, d, f, h$ ) components of the mean velocity in the midspan $(z / H=0)$ plane at indicated streamwise stations from the current LES case $S$ (dashed lines) and case $T$ (solid lines). The abscissa is the coordinate-shifted $y$ - axis adjusting for the hill profile. In frames showing profiles at more than one location, darker colors are used for farther downstream locations.

the third row of frames belong in either side of the location of maximum FPG at $x / H \approx$ -0.43 ; the last row of frames are pertinent to the pressure gradient turning adverse again at $x / H \approx-0.02$ and the consequent onset of separation and the separated flow. Profiles at several streamwise stations are shown in each frame, with darker colors used for farther downstream profiles. Additionally, to clearly show the evolving near-wall behaviour, local profiles of $\langle u\rangle$ and $\left\langle u^{\prime} u^{\prime}\right\rangle$ from Figs. 28 and 29 between streamwise stations $x / H=-2$ and $x / H=-0.26$ are also shown in Fig. 30 where a logarithmic scale is used for the abscissa. 
In all three figures, the profiles for cases $T$ and $S$ are shown by the solid and dashed lines, respectively.

At $x / H=-3$, mean profiles for both LESs are similar, showing little effect of the incoming turbulence for the case $T$ in Fig. 28. The slope of the $\langle u\rangle$ profiles right next to the wall decreases with downstream distance between $x / H=-3$ and -2 due to the APG. Owing to the incoming turbulence, profiles for case $T$ are fuller than for case $S$ at both stations $x / H=-2$ and -1.65 . The difference between the profiles for the two LESs persists in the APG region. In the upstream-side slope of the hill at $x / H=-1.65$, the $v-$ momentum increases due to an increase in mass transport caused by the presence of the hill. At all these stations, the mean profiles for the two cases collapse in the outer region. It is intriguing that despite the significant difference in the near-wall profiles between the two cases at the foot of the hill up to the point of a switch in pressure gradient at $x / H=-1.5$, separation onsets for the two cases are fairly close. Expectedly, in the first row of plots in Fig. 29, the turbulent stresses are higher throughout the boundary layer, specifically in the outer regions of the flow for case $T$. Initially, the $\left\langle u^{\prime} u^{\prime}\right\rangle$ component for case $T$ is larger than case $S$; the difference in magnitudes between the two cases initially increases and then decreases significantly in the FPG region. The differences between the profiles of $\langle u\rangle$ and $\left\langle u^{\prime} u^{\prime}\right\rangle$ for the two cases very close to the wall in the upstream-side APG region are more clearly seen in Fig. 30.

At $x / H=-1.35$, downstream of the initiation of the FPG, the difference between the profiles of the components of the mean velocity and the turbulent stresses for the two LESs decreases throughout the boundary layer; specifically, very close to the wall up to $\left(y-y_{0}\right) / H \approx 0.001$, profiles of all components of the mean velocity and turbulent stresses collapse on each other. In the outer region, the mean profiles for the two LESs collapse again for $\left(y-y_{0}\right) / H>0.1$. The deviation between the two cases is very apparent between these two regions. A near-wall peak appears for all components of the turbulent stresses at $x / H=-1.35$. A clear 'knee point' $[26,27]$ is apparent in the profile of the $\left\langle u^{\prime} u^{\prime}\right\rangle$ component for the case $T$ at this station. However, differences remain in the outer region for the turbulent stresses owing to the turbulent inflow condition for case $T$. Collapse of all profiles very close to the wall at $x / H=-1.35$, and the presence of the 'knee point' for case $T$ indicate the formation of an internal layer due to a switch in pressure gradient from adverse to favorable. Farther downstream, at $x / H=-1.11$ and -1, i.e., on either side of the hill curvature turning convex, no significant change is noticeable; with further evolution of the internal layer the difference between profiles for the two LESs decrease up to a greater distance away from the wall (see Fig. 30). The discrepancy in profiles of both $\langle u\rangle$ and $\langle v\rangle$ for the two LESs in the intermediate layer between the internal layer right next to the wall and the outer flow reduces with downstream distance. The difference is negligible by $x / H=-0.48$. The readjustment between the inner and outer flows is complete as the profiles for the two LESs collapse in both inner and the outer regions. At the streamwise stations shown in Fig. 28(e), profiles of $\langle u\rangle$ consist of a steep internal layer and the bulging outer flow; that the profiles of both LES cases collapse all throughout the boundary layer is clearly shown in Fig. 30. The difference in mean flow profiles for the two LESs down- 

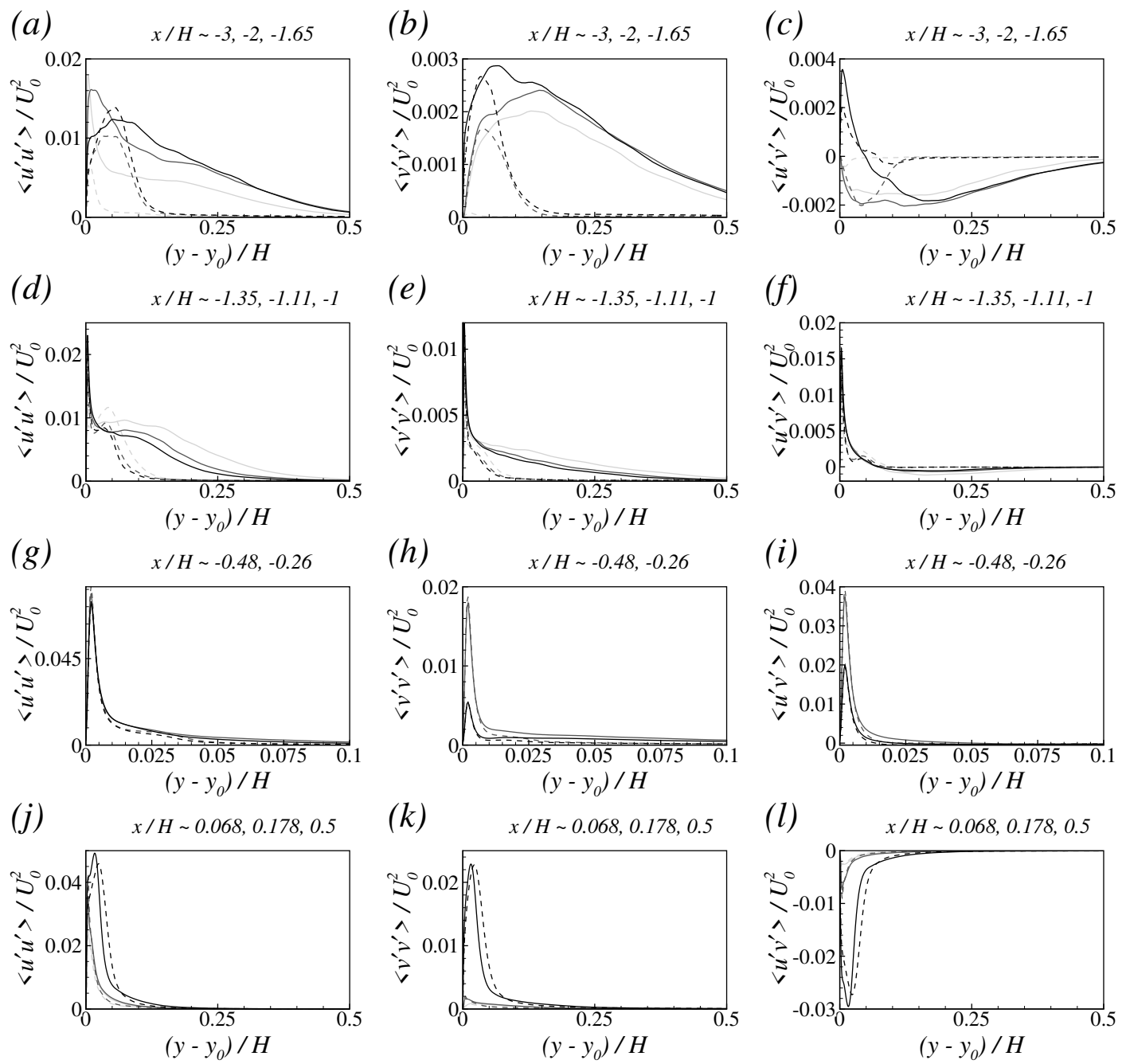

Fig. 29. Local profiles of the resolved turbulent stresses: $x$ - component $\left(\frac{\left\langle u^{\prime} u^{\prime}\right\rangle}{U_{0}^{2}}\right)$ in frames $a, d, g, j$, $y$ - component $\left(\frac{\left\langle v^{\prime} v^{\prime}\right\rangle}{U_{0}^{2}}\right)$ in frames $b, e, h, k$, and cross-stream component $\left(\frac{\left\langle u^{\prime} v^{\prime}\right\rangle}{U_{0}^{2}}\right)$ in frames $c, f, i, l$ in the midspan $(z / H=0)$ plane at the indiacted streamwise stations from the current LES case $S$ (dashed lines) and case $T$ (solid lines). The abscissa is the coordinate-shifted $y$-axis adjusted for the hill profile. In frames showing profiles at more than one location, darker colors are used for farther downstream locations.

stream of the crest of the hill is negligible up to the onset of separation. In Figs. 28(b), $(d)$ and $(f),\langle v\rangle>0$ and increases with downstream distance up to $x / H=-0.43$, i.e., the location of the peak FPG; downstream of the peak FPG, $\langle v\rangle$ reduces and ultimately switches sign downstream of the crest of the hill in Fig. 28(h).

For the profiles of $\left\langle u^{\prime} u^{\prime}\right\rangle$, the values are higher for case $T$ than for case $S$ in the outer region at all stations upstream of the crest of the hill (Fig. 30) This difference is due to 
(a)

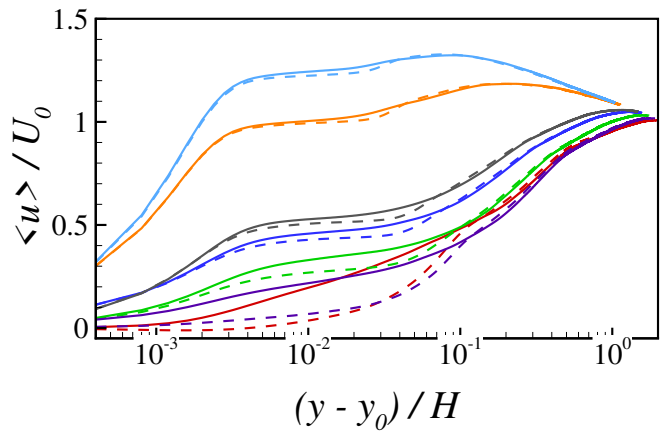

(b)

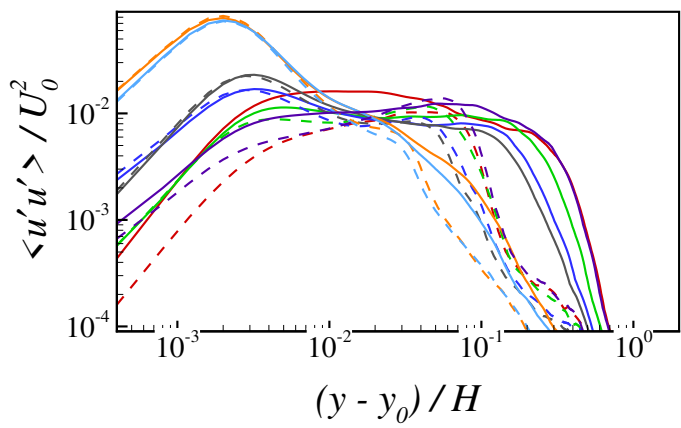

Fig. 30. Profiles of $(a)$ mean streamwise velocity $\left(\langle u\rangle / U_{0}\right)$ in Fig. 28(a, c,e), and $(b)$ resolved turbulent stress $\frac{\left\langle u^{\prime} u^{\prime}\right\rangle}{U_{0}^{2}}$ in Fig. 29(a,d,g) against coordinate-shifted $y$ - axis plotted in log scale at streamwise stations: $x / H=-2$ (red), $x / H=-1.65$ (violet), -1.35 (green), -1.11 (blue), -1 (black), -0.48 (orange), and -0.26 (light blue) from the LES case $S$ (dashed lines) and case $T$ (solid lines).

the incoming turbulence. However, the near-wall $\left\langle u^{\prime} u^{\prime}\right\rangle$ profiles for the two LESs collapse for $\left(y-y_{0}\right) / H<0.01$ downstream of $x / H=-1.35$. The outer peak in the profiles of $\left\langle u^{\prime} u^{\prime}\right\rangle$ in Fig. 29(g) disappears possibly due to an attenuation of turbulent fluctuations for case $T$ in the FPG region. However, the inner peaks of the resolved stresses keep increasing in magnitude up to the point of maximum FPG for all components of stresses. At $x / H \approx-0.26$, after crossing the location of the minimum pressure gradient, the resolved stresses decrease as the pressure gradient increases, and ultimately switch from favorable to adverse again at $x / H=-0.02$. In the APG region in the wake of the hill in the fourth row of Figs. 28 and 29, both mean velocity and turbulent stress profiles for the two cases collapse until the separation onset. Despite significant differences in the mean velocity and turbulent stresses at the foot of the hill at $x / H=-2$, after the development of the internal layer, and consequent readjustment between the inner and outer flows, all components of the mean velocity and turbulent stresses collapse for both LESs near the wall.

If the appearance of the internal layer and the consequent readjustments in the turbulent stresses were due to a change in surface curvature, one would expect the development of the internal layer to be related to the switches in curvature first turning concave and then from concave to convex in the upstream side of the hill in Fig. 27, as in the experiments of turbulent boundary-layer flow over a $2-D$ bump in [26] where the flow was of a similar nature $(\delta / H \approx 0.4$ in their case compared to 0.5 herein). The internal layer emerges downstream of the location of pressure-gradient switch from adverse to favorable at $x / H \approx-1.5$. Because the profiles of all components of the mean velocities and turbulent stresses for the two LESs initially collapse only very close to the wall and also in the outer wake region with an intermediate region where the discrepancies are significant, it appears 
that the internal layer develops somewhat independently of the outer flow, and is a consequence of the perturbations due a switch in the pressure gradient. Although the emergence of the internal layer might not to be explicitly related to the surface curvature effect, the downstream evolution of the pressure gradient is set by the curvature of the hill surface.

Farther downstream, after the zero-crossing point in streamwise pressure gradient distribution in Fig. 27, the flow experiences an APG. In the last row of frames in Figs. 28 and 29 , at $x / H=0.05$, the steepening of the profiles that lead to eventual separation is underway. Profiles have been also shown at $x / H=0.178$, just upstream of the separation onset in this plane for both LESs, and at $x / H=0.5$, i.e., in the separation region. The near-wall profiles for both LESs collapse fairly well up to $x / H=0.178$ in these plots. At the final station post separation, significant discrepancies reappear for the LESs for both mean velocity and turbulent stresses. In Fig. 12, the onset of separation for case $S$ is at $x / H \approx 0.2$, and for the case $T$ at $x / H \approx 0.24$, respectively. The slight difference between the two cases is due to the suppression of the separation in the APG region by the turbulent fluctuations in the outer flow for case $T$. However, the turbulent fluctuations in the outer flow for case $T$ have a more significant impact on the flow downstream of separation.
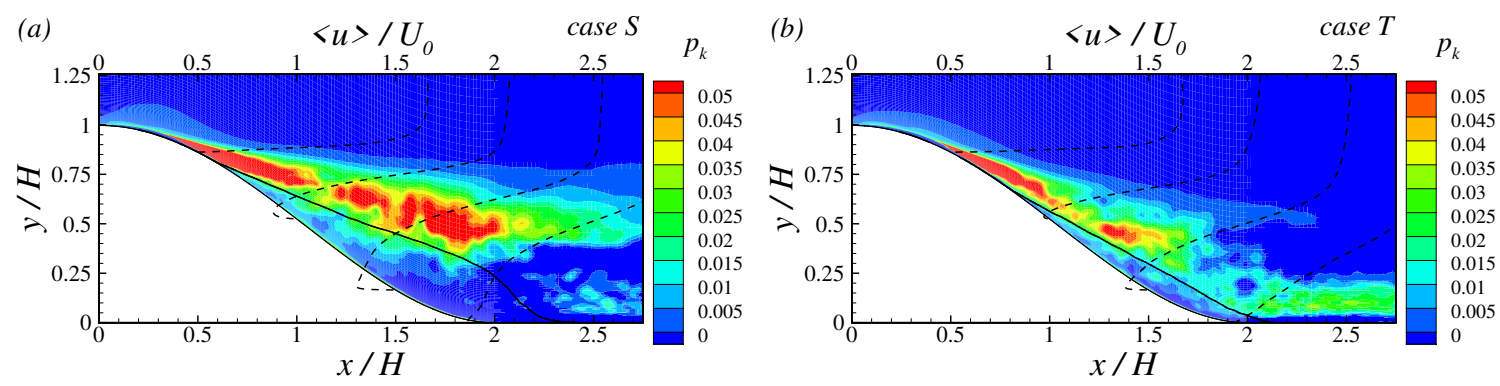

Fig. 31. Contours of the production of turbulent kinetic energy scaled by the reference length and velocity scales $\left(p_{k} \frac{H}{U_{0}^{3}}\right)$ plotted in the midspan $(z / H=0)$ plane from the current LES: $(a)$ case $S$ and (b) case $T$. Zero contour of the mean-streamwise velocity $(\langle u\rangle=0)$ is shown (solid line) to depict the mean recirculation region. Scaled streamwise velocity profiles $\left(\langle u\rangle / U_{0}\right)$ are also shown at $x / H=0.5,1,1.5$, and 2 by the coordinate-shifted dashed lines. The scale for $\langle u\rangle / U_{0}$ is shown at the top of the frame.

The production of $T K E$ is expressed as,

$$
p_{k}=-\left\langle u_{i}^{\prime} u_{j}^{\prime}\right\rangle \frac{\partial\left\langle u_{i}\right\rangle}{\partial x_{j}}
$$

Contours of $p_{k}$ scaled by the reference velocity and length scales in the midspan plane are plotted in Fig. 31 for both LESs downstream of the crest of the hill depicting the separated flow and early recovery regions. In the same plots, coordinate-shifted mean streamwise velocity profiles are shown at four streamwise stations in the separated flow region along with the $\langle u\rangle=0$ contour to depict the recirculation layer. Expectedly, contours 
of $p_{k}$ resemble the $T K E$ contours for the two cases shown in Fig. 16. As for $T K E$ contours in Fig. 16, a patch of high $p_{k}$ is obtained very close to the wall just upstream of the separation onset for both cases. From its expression, $p_{k}$ is high where either the turbulent stresses are large or the velocity gradient is large, or where both terms are significant. High production of $T K E$ takes place just outside the flow recirculation region for both cases. Additionally, the $\langle u\rangle$-profiles show that high $T K E$ production is associated with the highvelocity-gradient regions of the flow. Close to the wall the dominant gradient term, $\frac{\partial\langle u\rangle}{\partial y}$ is low; the gradient drastically increases away from the wall above the recirculating flow for both cases. The turbulent fluctuations in the separated shear layer or the outer flow for case $T$ increase the turbulent stresses there, consequently increasing the velocity gradient of the outer flow. This results in a high $T K E$ production in the thinner separated shear layer close to the wall depicted by the $p_{k}$ contours for case $T$ in Fig. 31(b). High velocity gradient closer to the wall ensures correct capturing of the recirculation layer and the separated shear layer for case $T$. Additionally, the turbulent fluctuations in the separated shear layer suppress the recirculation layer by transporting momentum. On the other hand, after the initiation of separation for case $S$, because of low turbulent fluctuations in the outer flow, the velocity gradient is relatively low compared to case $T$. Therefore, high $p_{k}$ is obtained over a locally thicker layer for case $S$, which is also reflected in the locally thicker patch of high $T K E$ downstream of separation in Fig. 16. Due to low near-wall gradient in the velocity, the recirculation region is thicker for this case, and the separation is more akin to laminar separation. This also results in late reattachment and thereby, late recovery of the flow. 


\section{Discussion \& Conclusions}

Two LES simulations were performed to study the separational flow behind a smoothwall axisymmetric hill at a high Reynolds number (Reynolds number w.r.t. hill height is $\left.R e_{H}=130,000\right)$. Their results are compared in extensive detail with experiments of the same flow reported in $[3,4,6,38]$. The only difference between the LESs is the boundary condition applied at the inflow of the computational domain. One of the LESs (case $S$ ) was performed with the mean velocity profile from the experiments used as the inflow boundary condition. For the other LES (case $T$ ), unsteady velocity profiles from a precursor LES are used. In the precursor LES, the time-averaged streamwise velocity field is forced to match the experimental profile. The LESs are performed on a grid with 45 million cells, which may be considered nominal for this flow. Grid stretching in all three directions ensures that the relevant regions of the flow are adequately resolved (Fig. 3). The ratio between the mean SGS eddy viscosity and a computed total eddy viscosity in Figs. 5 and 6 show that the contribution of SGS eddy viscosity towards the computed Reynolds stresses is negligible, implying that both LESs are accurate. The simulations presented herein provide a guidance for baseline resolution required for a resolved LES computation for high-Re flows on the widely used CFD program OpenFOAM [31].

The motivation behind performing the LESs was to generate reliable data for the purpose of formulating data-based turbulence models for separational flows. In that regard, the cost of computing the case $S$ is lower than case $T$. Based on experience of simulating this flow on coarser grids ( $\approx 15$ million cells), it was argued in [15] that the effects of the incoming turbulence on the flow separation could be weak for this flow. Later, in [21], using steady laminar and turbulent velocity profiles as inlet conditions found some influence of the inlet boundary condition on the separational flow dynamics, albeit at a much lower Reynolds number. Comparison of the time-averaged LES results with experiment shows that flow statistics from case $T$ are in good agreement with the experiments, while for case $S$, several discrepancies are observed. Upstream of separation the $C_{p}$ distribution showed good agreement with the experiment for both cases (see Figs. 10 and 11); however, downstream of separation, a longer and flatter plateau is obtained for case $S$, implying a larger and thicker recirculation layer, and significantly delayed reattachment, as shown in Fig. 12. For case $T$, the recirculation-layer thickness is in good agreement with the experiment in most of the separation region with a slightly delayed reattachment. As in [16], flow topology maps were studied by plotting the mean streamlines at various wall-parallel planes in Figs. 13 and 15. These figures depict the mean separation dynamics in great detail. In total four distinct nodes and four distinct saddle points are obtained for both LESs which is in accordance with the flow topology reported in [16]. Except in a thin narrow patch of reattached flow downstream of the initial separation in Fig. 13, good agreement between the flow topology map and $T K E$ contours at all wall-normal heights for the case $T$ and the high-resolution LESs in [16] is evident in Fig. 15. As this reattached flow region is absent for case $S$, this is possibly due to the under-resolved incoming turbulence for case $T$. On the other hand, for case $S$, the flow topology maps show large and thick focal vortices for 
the case $S$. The instantaneous separational flow field is highly intermittent and only bear some resemblance with the mean flow dynamics (Fig. 25), and is sensitive to several computational attributes, such as, the incoming turbulence, grid resolution and stretching, and so forth. Such sensitivity makes the prediction of the separated flow challenging.

To realize the connection between the mean flow recovery in the lee-side of the hill and the upstream separational flow, mean streamwise vorticity $\left(\left\langle\omega_{x}\right\rangle\right)$ is useful as it depicts secondary motions. Isosurfaces of $\left\langle\omega_{x}\right\rangle$ in Figs. 17 and 18 reveal several layers of vorticity with alternating sense in the $y$ direction. Furthermore, in the separation region, there are three main sources of vorticity, namely, re-oriented vorticity of the upstream boundary layer, vorticity in the recirculation layer consisting of the vorticities from the focal nodes and the reverse flow, and the vorticity ejected from the separation node. Contour plots of $\left\langle\omega_{x}\right\rangle$ in two cross-stream planes, one in the separation region, and the other, further downstream post-reattachment in Fig. 19 demonstrate that the upstream boundary-layer vorticity and the vortices ejected from the separation node dominate the vorticity in the near wake. The vorticities ejected from the focal nodes for the case $S$ are stronger that extend up to a thicker recirculation layer. As the flow recovers farther downstream in an ambience of an APG, results for case $T$ are found to be in good agreement with the experiment in a crossstream plane at a chosen stream-wise station. Both the contours of $T K E$ and the secondary motions in the cross-stream plane at $x / H \approx 3.63$ for case $S$ are erroneous in Fig. 20. While the $T K E$ is overpredicted around the midspan plane, an additional pair of inner vortices strengthen, followed by dissipation along with the outer vortex pair; vortices in either side of the midspan plane have the same sign. As a consequence, wall-normal profiles of the stream-wise and span-wise components of the mean velocity in Fig. 22, along with the $T K E$ and $\left\langle u^{\prime} v^{\prime}\right\rangle$ components of the resolved turbulent stresses in Fig. 23, show significant overprediction close to the midspan plane for the case $S$; the agreement improves farther away from the midspan plane. For case $T$ on the other hand, good agreement with the experiment is obtained at all spanwise stations. Spectra of the stream-wise and span-wise fluctuation velocities at three wall-normal heights at this station are also in good agreement with the experiment in Fig. 26.

The instantaneous flow fields of the separation and flow-recovery regions were analyzed by plotting the fluctuating component of the stream-wise velocity field at two wall-parallel planes extracted at chosen heights above the bottom wall in Fig. 24 for the case $T$. Long stream-wise streaky structures in the wake-side of the hill extend downstream in to the wake and recovery regions of the flow above the recirculation layer. Both high-speed and low-speed streaks w.r.t. to the time-averaged flow appear in the separated flow region in different orientations, which sustain and consequently wash away in the wake of the hill. These streaky structures are intermittent, and show imprints on the near-wall flow field. The plots for the instantaneous streamlines in Fig. 25 at the same time instants as in Fig. 24, show that the patterns appearing in the mean flow fields are inherent in the instantaneous flow, although their appearances are highly irregular. Interestingly, comparison of Fig. 24 and 25 shows that the low-speed streaky structures are associated with the instantaneous focal vortices. These instantaneous flow fields despite being largely different, provide a 
very complex, yet clear time-mean flow pattern discussed earlier.

Despite significant differences between the two LESs, specifically downstream of separation as shown in Sec. 3, the onset of separation is very close for the two simulations. The $C_{p}$ distribution for the LESs are in good agreement with each other in Figs. 10 and 11 upstream of separation. Figure 27(b) shows that the stream-wise pressure gradient for the two cases are also very similar both upstream and downstream of separation. Also, the integral parameters for the boundary layer at the lower wall show good match between the two LESs upstream of separation, implying that the effect of the pressure gradient is experienced throughout the entire boundary layer. The curves for the two cases diverge only downstream of separation. Inspite of these similarities between the two LESs, the local profile of the mean streamwise velocity is significantly fuller, and the resolved turbulent stresses are higher close to the wall at the foot of the hill (Figs. 28 and 29, respectively) for case $T$. However, as the pressure gradient switches from adverse to favorable in the upstream side of the hill, an internal layer [26] emerges and consequently all components of the mean velocity and trubulent stresses collapse for the two cases close to the wall (Fig. 30). The mean velocity profiles also match in the outer wake region of the boundary layer. Initially, discrepancies between the two LESs are significant in the intermediate region between the internal layer and outer wake regions; they decrease with downstream distance, and quickly become negligible as the flow in the intermediate region readjusts for both cases. Following the formation of the internal layer, the near-wall profiles for the two cases are the same as the pressure gradient turns adverse just upstream of the hill crest and ultimately leads to separation. The role of the incoming turbulence for case $T$ in this flow regime is to slightly delay the separation onset. The incoming turbulence, however, plays a significant role downstream of separation. The turbulence fluctuations in the outer flow for the case $T$ increases the near-wall velocity gradient, thereby resulting in a thinner recirculation region and separated shear layer, in good agreement with the experiment. Due to lower turbulent fluctuations in the outer flow, the velocity gradients are lower, resulting in a thicker recirculation layer with laminar-like separation. The results show that correct capturing of the near-wall internal layer is essential for predicting the separation onset for the smooth-wall separation. The incoming turbulence is essential for the correct prediction of the downstream separation dynamics, reattachment, and recovery of this flow. 


\section{References}

[1] Ling J, Kurzawski A, Templeton J (2016) Reynolds averaged turbulence modelling using deep neural networks with embedded invariance. J Fluid Mech 807:155-166.

[2] Duraisamy K, Iaccarino G, Xiao H (2019) Turbulence modeling in the age of data. Annu Rev Fluid Mech 51:357-377.

[3] Simpson RL, Long CH (2001) Study of vortical separation from an axisymmetric hill. TSFP DIGITAL LIBRARY ONLINE (Begel House Inc.), , .

[4] Ma R, Simpson RL (2005) Characterization of turbulent flow downstream of a threedimensional axisymmetric bump. Tsfp Digital Library Online (Begel House Inc.), ,

[5] Byun G (2005) Sturcture of Three-Dimensional Separated Flow on Symmetric Bumps. Ph.D. thesis. Virginia Tech, .

[6] Byun G, Simpson RL (2006) Structure of three-dimensional separated flow on an axisymmetric bump. AIAA J 44(5):999-1008.

[7] Ishihara T, Hibi K, Oikawa S (1999) A wind tunnel study of turbulent flow over a three-dimensional steep hill. J Wind Eng Ind Aerodyn 83(1-3):95-107.

[8] Wang C, Jang YJ, Leschziner MA (2004) Modelling two-and three-dimensional separation from curved surfaces with anisotropy-resolving turbulence closures. Int J Heat Fluid Fl 25(3):499-512.

[9] Fureby C, Alin N, Wikström N, Menon S, Svanstedt N, Persson L (2004) Large eddy simulation of high-reynolds-number wall bounded flows. AIAA journal 42(3):457468.

[10] Davidson L, Dahlström S (2005) Hybrid les-rans: Computation of the flow around a three-dimensional hill. Engineering Turbulence Modelling and Experiments 6 (Elsevier), , pp 319-328.

[11] Persson T, Liefvendahl M, Bensow RE, Fureby C (2006) Numerical investigation of the flow over an axisymmetric hill using les, des, and rans. J Turbul (7):N4.

[12] Patel N, Menon S (2007) Structure of flow separation and reattachment behind an axisymmetric hill. $J$ Turbul (8):N36.

[13] Tessicini F, Li N, Leschziner MA (2007) Large-eddy simulation of three-dimensional flow around a hill-shaped obstruction with a zonal near-wall approximation. Int J Heat Fluid Fl 28(5):894-908.

[14] Visbal M, Rizzetta D, Mathew J (2007) Large eddy simulation of flow past a 3-d bump. 45th AIAA Aerospace Sciences Meeting and Exhibit, , p 917.

[15] Krajnović S (2008) Large eddy simulation of the flow over a three-dimensional hill. Flow, Turbulence and Combustion 81(1-2):189-204.

[16] Garcia-Villalba M, Li N, Rodi W, Leschziner MA (2009) Large-eddy simulation of separated flow over a three-dimensional axisymmetric hill. J Fluid Mech 627:55-96.

[17] Jamal T, Chitta V, Walters DK (2021) Numerical simulation of a three-dimensional axisymmetric hill: Performance evaluation of hybrid rans-les models. J Fluids Eng .

[18] Jamal T, Walters DK (2016) Simulation of a 3d axisymmetric hill: Comparison of 
rans and hybrid rans-les models. ASME 2016 Fluids Engineering Division Summer Meeting collocated with the ASME 2016 Heat Transfer Summer Conference and the ASME 2016 14th International Conference on Nanochannels, Microchannels, and Minichannels (American Society of Mechanical Engineers), , pp V01BT25A010V01BT25A010.

[19] Bose R, Yeo D (2021) Simulations of flow over an axisymmetric hill. https://doi.org/ https://doi.org/10.6028/NIST.TN.2141.

[20] Walters DK, Bhushan S, Alam M, Thompson DS (2013) Investigation of a dynamic hybrid rans/les modelling methodology for finite-volume cfd simulations. Flow, turbulence and combustion 91(3):643-667.

[21] García-Villalba M, Wissink JG, Rodi W (2010) Influence of the approach boundary layer on the flow over an axisymmetric hill at a moderate reynolds number. $J$ Turbul (11):N8.

[22] Smits A, Wood D (1985) The response of turbulent boundary layers to sudden perturbations. Annu Rev Fluid Mech 17(1):321-358.

[23] Blackwelder RF, Kovasznay LS (1972) Large-scale motion of a turbulent boundary layer during relaminarization. J Fluid Mech 53(1):61-83.

[24] Narasimha R, Sreenivasan K (1973) Relaminarization in highly accelerated turbulent boundary layers. J Fluid Mech 61(3):417-447.

[25] Gillis J, Johnston J (1983) Turbulent boundary-layer flow and structure on a convex wall and its redevelopment on a flat wall. J Fluid Mech 135:123-153.

[26] Baskaran V, Smits A, Joubert P (1987) A turbulent flow over a curved hill part 1. growth of an internal boundary layer. J Fluid Mech 182:47-83.

[27] Baskaran V, Smits A, Joubert P (1991) A turbulent flow over a curved hill. part 2. effects of streamline curvature and streamwise pressure gradient. J Fluid Mech 232:377-402.

[28] Lilly DK (1992) A proposed modification of the germano subgrid-scale closure method. Physics of Fluids A: Fluid Dynamics 4(3):633-635.

[29] Smagorinsky J (1963) General circulation experiments with the primitive equations: I. the basic experiment. Monthly weather review 91(3):99-164.

[30] Germano M, Piomelli U, Moin P, Cabot WH (1991) A dynamic subgrid-scale eddy viscosity model. Physics of Fluids A: Fluid Dynamics 3(7):1760-1765.

[31] Weller HG, Tabor G, Jasak H, Fureby C (1998) A tensorial approach to computational continuum mechanics using object-oriented techniques. Comput Phys 12(6):620-631.

[32] Davidson L (2009) Large eddy simulations: how to evaluate resolution. Int J Heat Fluid Fl 30(5):1016-1025.

[33] Spalding DB (1961) A single formula for the "law of the wall" .

[34] Choi H, Moin P (1994) Effects of the computational time step on numerical solutions of turbulent flow. J Comput Phys 113(1):1-4.

[35] Durbin PA, Pettersson Reif BA (2011) Statistical theory and modeling for turbulent flows (John Wiley \& Sons), .

[36] Pierce CD, Moin P (2001) Progress-variable approach for large-eddy simulation of 
turbulent combustion. Ph.D. thesis. Citeseer, .

[37] Counihan JO (1975) Adiabatic atmospheric boundary layers: a review and analysis of data from the period 1880-1972. Atmospheric Environment (1967) 9(10):871-905.

[38] Simpson RL, Long CH, Byun G (2002) Study of vortical separation from an axisymmetric hill. Int J Heat Fluid Fl 23(5):582-591. https://doi.org/https://doi.org/10.1016/ S0142-727X(02)00154-6

[39] Hunt JCR, Abell CJ, Peterka JA, Woo H (1978) Kinematical studies of the flows around free or surface-mounted obstacles; applying topology to flow visualization. $J$ Fluid Mech 86(1):179-200. 